\title{
On Elimination of Variables in the Study of Singularities in Positive Characteristic
}

ANGÉlica Benito \& ORLANDo E. Villamayor U.

ABSTRACT. The objective of this paper is to discuss some invariants of singularities of algebraic schemes over fields of positive characteristic, and to show their usefulness for the simplification of singularities. We focus here on invariants that arise in an inductive manner, namely, by successive elimination of variables. When applied to a hypersurface singularity, they lead to a refinement of the notion of multiplicity. The Weierstrass Preparation Theorem allows us to express the equation defining the hypersurface as a polynomial equation; in this case, the new invariants can be defined, in some way, from the coefficients of this polynomial, and hence this theorem enables us to define invariants in one variable fewer.

In this paper, we present a generalized form of the Weierstrass Preparation Theorem that enables us to eliminate several variables at once, and to define invariants in this more general setting.

This leads to the definition of inductive invariants that refine the multiplicity in the hypersurface case. In addition, they provide a refinement of the Hilbert-Samuel stratification for the non-hypersurface case.

Finally, the paper includes some applications of these invariants to the open problem of resolution of singularities.

\section{CONTENTS}

1. Introduction

2. Some Basics and an Overall View of the Paper 362

3. Weak Equivalence 379

4. The Slope of a Hypersurface and the Weak Equivalence 381

5. Slope of a Rees Algebra, $(d-1)$-dimensional H-function $(\tau \geq 1) \quad 389$

6. Simplified Presentations, $(d-r)$-dimensional H-functions $(\tau \geq r) \quad 394$

7. Main Properties of $\mathrm{H}$-functions and Proof of Theorem 2.13 


\section{INTRODUCTION}

1.1. In 1964, Hironaka proved resolution of singularities over fields of characteristic zero in his celebrated paper $[28,29]$. Herein, he first quantified the singularities making use of the Hilbert-Samuel function. More precisely, a singular variety $X$ is stratified in accordance with the Hilbert-Samuel function attached to its points. Roughly speaking, each stratum is given by the points that have the same function.

Hironaka proved that this invariant (the worst Hilbert-Samuel function corresponding to points in $X$ ) can be improved by applying suitable transformations, and that resolution of singularities is achieved by successive applications of this procedure.

The significant step in his proof consists in proving that the worst HilbertSamuel function can be improved. To this end, he makes use of local arguments, and assumes that locally at a given point $x \in X$, there is an immersion $X \subset W$, where $W$ is a regular variety. If $I$ denotes the ideal defining $X$ at $\mathcal{O}_{W, x}$ he constructs, at the completion $\hat{\mathcal{O}}_{W, x}$, finitely many elements $\left\{h_{1}, \ldots, h_{s}\right\}$ and positive integers $\left\{n_{1}, \ldots, n_{s}\right\}$, so that each $h_{i}$ has multiplicity $n_{i}$ at $\hat{\mathcal{O}}_{W, x}$, and the completion of the Hilbert-Samuel stratum is given by

$$
\mathcal{H}=\bigcap\left\{w \in W^{\prime} \mid \operatorname{ord}_{w}\left(h_{i}\right) \geq n_{i}\right\},
$$

where $W^{\prime}=\operatorname{Spec}\left(\hat{\mathcal{O}}_{W, x}\right)$. In particular, a regular center $Y$ is locally included in the Hilbert-Samuel stratum of $X \subset W$ if and only if its pull-back at $W^{\prime}$, say $Y^{\prime}$, is an equimultiple center for each hypersurface defined by $h_{i}=0$. He shows that the so-called idealistic exponent (e.g., $\left\{h_{1}, \ldots, h_{s}\right\}$ and $\left.\left\{n_{1}, \ldots, n_{s}\right\}\right)$ can be chosen so that if $\left\{h_{1}^{(1)}, \ldots, h_{s}^{(1)}\right\}$ denote the strict transforms at $W^{\prime}(1)$, obtained by blowing up $Y^{\prime}$, then the pullback to $W^{\prime}(1)$ of the Hilbert stratum of the strict transform of $X$ is defined by

$$
\mathcal{H}^{(1)}=\bigcap\left\{w \in W^{\prime(1)} \mid \operatorname{ord}_{w}\left(h_{i}^{(1)}\right) \geq n_{i}\right\} .
$$

In addition, Hironaka shows that $\left\{h_{1}, \ldots, h_{s}\right\}$ and $\left\{n_{1}, \ldots, n_{s}\right\}$ can be chosen so that this condition holds after one applies any sequence of blow-ups at centers included in the Hilbert-Samuel stratum.

This argument provides a local reduction to the hypersurface case, and the advantage is that the law of transformation of a hypersurface is easy to handle.

A resolution of an idealistic exponent consists of the construction of a sequence of monoidal transformations so that the final transform, for instance, $\mathcal{H}^{(r)}$, where

$$
\mathcal{H}^{(r)}=\bigcap\left\{w \in W^{\prime(r)} \mid \operatorname{ord}_{w}\left(h_{i}^{(r)}\right) \geq n_{i}\right\},
$$

is empty. This would lead to an improvement of the worst Hilbert-Samuel stratum, at least locally above $x \in X$. 
In addition, Hironaka assigns to an idealistic exponent the notion of maximal contact, consisting in part of a regular system of parameters of $\hat{\mathcal{O}}_{W, x}$ (e.g., $\left.\left\{z_{1}, \ldots, z_{\tau}\right\}\right)$ that is obtained from the data. The regular variety $\left\{z_{1}=0, \ldots, z_{\tau}=\right.$ $0\}$ has the property that it contains the Hilbert-Samuel stratum $\mathcal{H}$, for instance,

$$
\mathcal{H} \subseteq\left\{z_{1}=0, \ldots, z_{\tau}=0\right\}=\bigcap\left\{w \in W^{\prime} \mid \operatorname{ord}_{w}\left(z_{i}\right) \geq 1\right\},
$$

and that the inclusion is preserved by the previous kind of monoidal transformations.

This inclusion is crucial in Hironaka's induction on the dimension of the ambient space, as it enables him to construct a resolution of an idealistic exponent.

Let us indicate that this construction of the idealistic exponents at the completion (and of their resolution at this setting) was sufficient to obtain the nonconstructive proof presented in $[28,29]$.

In 1975, in [23], Giraud partially extended the previous results to the case of positive characteristic: given a point $x \in X$, and an immersion $X \subset W$ in a regular variety, he constructed at $\hat{\mathcal{O}}_{W, x}$ a presentation (see [23, Definition 3.1]):

- An idealistic exponent given by elements $h_{1}, \ldots, h_{s}$ and integers $n_{1}$, $\ldots, n_{s}$, such that each $h_{i}$ has multiplicity $n_{i}$ at $\hat{\mathcal{O}}_{W, x}$, and the HilbertSamuel stratum is $\mathcal{H}=\bigcap\left\{w \in W^{\prime} \mid \operatorname{ord}_{w}\left(h_{i}\right) \geq n_{i}\right\}$, where, again, $W^{\prime}=\operatorname{Spec}\left(\hat{\mathcal{O}}_{W, x}\right)$;

- A part of a regular system of parameters $\left\{z_{1}, \ldots, z_{\tau}\right\}$ of $\hat{\mathcal{O}}_{W, x}$;

- Elements $f_{1}, \ldots, f_{\tau}$ of order $p^{e_{i}}$, certain powers of the characteristic, such that, if the base field is perfect, then $\operatorname{In}_{x}\left(f_{i}\right)=Z_{i}^{p^{e_{i}}} \in \operatorname{gr}\left(\hat{\mathcal{O}}_{W, x}\right)$, where $Z_{i}=\operatorname{In}_{x}\left(z_{i}\right)$.

In this setting, the polynomials $f_{j}$ mimic the role of the maximal contact given by Hironaka, since the pullback of the Hilbert-Samuel stratum fulfills

$$
\mathcal{H} \subseteq \bigcap\left\{w \in W^{\prime} \mid \operatorname{ord}_{w}\left(f_{i}\right) \geq p^{e_{i}}\right\} .
$$

Moreover, if $Y$ is a regular subvariety included in the Hilbert-Samuel stratum, and $Y^{\prime}$ is as before, Giraud proves that the previous inclusion is stable under a blow-up along $Y^{\prime}$, for instance,

$$
\mathcal{H}^{(1)} \subseteq \bigcap\left\{w \in W^{\prime}(1) \mid \operatorname{ord}_{w}\left(f_{i}^{(1)}\right) \geq p^{e_{i}}\right\},
$$

where the $f_{i}^{(1)}$ are the strict transforms of the $f_{i}$ at $W^{\prime}(1)$.

Let us indicate that the previous elements $z_{1}, \ldots, z_{\tau}$ are given with prescribed properties: the initial forms $Z_{i}=\operatorname{In}_{x}\left(z_{i}\right)$ describe the ridge of the tangent cone, which is a subspace included in $\mathbb{T}_{X, x}$. In addition, Giraud adds to this data a formally smooth morphism from $W^{\prime}$ to a formally smooth scheme of dimension $d-\tau$. This morphism is in some natural way transversal to the idealistic exponent, and this transversality is preserved by blow-ups that are defined as above. 
In 1977 , Hironaka showed that $\left\{h_{1}, \ldots, h_{s}\right\}$ and $\left\{n_{1}, \ldots, n_{s}\right\}$ can be constructed, within the conditions introduced by Giraud, but now at the henselization of $\mathcal{O}_{W, x}$ (see [33]). This is a result of Aroca that applies to varieties defined over perfect fields, and allows one to show that all the previous data hold at a suitable étale neighborhood of $x \in W$. Therefore, Giraud's presentations can be developed within the class of schemes of finite type over a perfect field.

The results in this paper are related in many ways to those of Giraud, although we also make use of the existence of idealistic exponents in étale topology. Our motivation is Hironaka's treatment of idealistic exponents in characteristic zero. In this case, he constructs a maximal contact variety of dimension $d-\tau$. Moreover, he defines a new idealistic exponent in this smaller-dimensional regular variety. It is in terms of these restricted data that he defines a function, called here $\mathrm{H}$-ord ${ }^{(d-\tau)}$ (see [33, Proposition 8]). We also refer to Theorem 11.1 and Definition 11.8 in [35] for Hironaka's definition of these functions in the context of local analytic spaces.

In this paper, we reproduce, at least partially, this strategy in characteristic $p$, with some essential differences: we make use, as in Giraud's approach, of suitable transversal projections from a regular variety of dimension $d$ to one of dimension $d-\tau$, together with a form of elimination of variables. A second ingredient, also crucial for the construction of our extended function $\mathrm{H}$-ord ${ }^{(d-e)}$, will be the Generalized Preparation Theorem (see Theorem 6.5). This theorem will encompass all the data in Giraud's presentations, and will allow us to improve the form of Giraud's functions $f_{i}$.

In fact, the transversal projection and the preparation theorem enable us to present the $f_{i}$ as polynomials. Moreover, the $f_{i}$ are of the form

$$
f_{i}\left(z_{i}\right)=z_{i}^{p^{e_{i}}}+a_{1}^{(i)} z_{i}^{p^{e_{i}-1}}+\cdots+a_{p^{e_{i}}}
$$

where the $a_{j}^{(i)}$ now involve only $d-\tau$-variables, and $\operatorname{In}_{x}\left(z_{i}\right)=Z_{i}, 1 \leq i \leq \tau$, define the ridge of the tangent cone at the given point. We will show that the presentations given in this form are also stable under monoidal transformations.

Thus, in our approach, the notion of restriction is replaced by that of projections. This is done in such a way that, when specialized to characteristic zero, we recover Hironaka's original $\mathrm{H}$-ord ${ }^{(d-\tau)}$ function.

As indicated, the invariants to be introduced here will be obtained by successive elimination of variables. They will generalize, at least for the case of elimination of one variable, invariants that were already treated in pioneering works of Cossart and Moh. This will also provide a refinement of the Hilbert-Samuel stratification for a scheme of finite type over a perfect field.

Finally, in Section 7, some numerical conditions involving these invariants will be discussed. We will ultimately show that, when these conditions hold, the worst Hilbert-Samuel stratum can be simplified by means of permissible transformations (blow-ups along centers included in a Hilbert-Samuel stratum). 
1.2. The form of elimination to be considered makes use of the Weierstrass Preparation Theorem. Fix a closed point $x \in X$, where $X$ denotes a hypersurface embedded in a smooth $d$-dimensional ambient scheme over a perfect field $k$ (e.g., $\left.V^{(d)}\right)$. Let the multiplicity of $X$ at $x$ be $n$. The Weierstrass Preparation Theorem asserts that, at a suitable étale neighborhood of $V^{(d)}$ at $X$, one can define a smooth morphism (e.g., $V^{(d)} \stackrel{\beta}{\longrightarrow} V^{(d-1)}$ ) so that the ideal of the hypersurface $X$ is spanned by a monic polynomial, for instance,

$$
f\left(z_{1}\right)=z_{1}^{n}+a_{1} z_{1}^{n-1}+\cdots+a_{n} \in \mathcal{O}_{V^{(d-1)}}\left[z_{1}\right] .
$$

Here, $\left\{z_{1}=0\right\}$ is a section of $\beta$, the coefficients lie in the smooth scheme $V^{(d-1)}$, and $\left\{z_{1}\right\}$ can be extended to a regular system of parameters in $\mathcal{O}_{V^{(d)}}, x$. These coefficients involve one variable less, so there is a form of elimination of $z_{1}$ in this approach. Despite the fact that such a polynomial is not intrinsic to $x \in X$, there is significant information of the singularity encoded in the coefficients, used here to define some invariants of the singularity $(X, x)$.

The $\tau$-invariant at a point $x \in X$, defined in terms of the ridge of the tangent cone, is a positive integer that indicates the number of variables that can be naturally eliminated, at least for the purpose of simplifying the singularity. We present a generalized form of the Weierstrass Preparation Theorem, which fits better in this frame, in which $\tau$ variables are to be eliminated $(\tau \geq 1)$. To illustrate this fact, assume that $\tau \geq 2$, in this case this invariant, indicates that one can eliminate two variables simultaneously. The usual Weierstrass Preparation Theorem would provide a smooth morphism at an étale neighborhood of $x$ (e.g., $V^{(d)} \stackrel{\beta}{\longrightarrow} V^{(d-1)}$ ), together with two monic polynomials, for example,

$$
f_{1}\left(z_{1}\right)=z_{1}^{n}+a_{1} z_{1}^{n-1}+\cdots+a_{n} \in \mathcal{O}_{V^{(d-1)}}\left[z_{1}\right],
$$

and

$$
f_{2}\left(z_{2}\right)=z_{2}^{m}+b_{1} z_{2}^{m-1}+\cdots+b_{m} \in \mathcal{O}_{V^{(d-1)}}\left[z_{2}\right] .
$$

The drawback of this outcome is that the coefficients of both polynomials lie in $V^{(d-1)}$, whereas we would like to eliminate simultaneously the two variables $z_{1}$ and $z_{2}$. We circumvent this difficulty by presenting a suitable generalization of the Weierstrass Preparation Theorem, which enables us to construct a smooth morphism (e.g., $V^{(d)} \stackrel{\beta}{\longrightarrow} V^{(d-2)}$ ), defined at an étale neighborhood of $x$, so that the ideal of the two equations can be expressed by monic polynomials of the form

$$
f_{1}\left(z_{1}\right)=z_{1}^{n}+a_{1} z_{1}^{n-1}+\cdots+a_{n} \in \mathcal{O}_{V^{(d-2)}}\left[z_{1}\right]
$$

and

$$
f_{2}\left(z_{2}\right)=z_{2}^{m}+b_{1} z_{2}^{m-1}+\cdots+b_{m} \in \mathcal{O}_{V^{(d-2)}}\left[z_{2}\right] .
$$


Here, $z_{1}$ and $z_{2}$ can be chosen so that $z_{1}=0$ and $z_{2}=0$ are two different sections of $\beta$, and $\left\{z_{1}, z_{2}\right\}$ can be extended to a regular system of parameters in $\mathcal{O}_{V^{(d)}, x}$ (see Proposition 6.1).

The advantage of this new formulation over the previous one is that the coefficients of both polynomials lie in a smooth scheme of dimension $d-2$ (involving two variables fewer). Moreover, when Hironaka's invariant is $r$, the generalized Weierstrass Preparation Theorem (Theorem 6.5) allows us to find $r$ monic polynomials, all with coefficients in a smooth scheme of dimension $d-r$ (involving $r$ variables fewer).

Some applications of this form of the Preparation Theorem will be addressed in the last part of Section 6 and Section 7. There, we extend the main results in [6], in particular, the definition of the H-functions (see Definition 6.10), treated in the cited paper for the case $\tau=1$. These $H$-functions will be used to formulate the notion of strong monomial case (Definition 7.1), and also to show that the simplification of singularities is possible in such case (see Theorem 7.3). The latter is a natural extension of [6, Theorem 8.14].

Another application of the generalized Preparation Theorem is the resolution of singularities of two-dimensional schemes (Theorem 7.4) by blowing up at regular centers. This result is an extension of the resolution of surfaces embedded in three-dimensional spaces, as was proved in [7].

The notion of invariant, as used in Hironaka's work, has a very precise meaning and special features. This will be discussed at the beginning of the next sections. These features will also hold for those invariants introduced in this work. In particular, they will inherit properties as that of equivariance.

Let us indicate that the invariants attached to a polynomial at a point in Theorem 4.10 and Corollary 4.11 are also treated by Cossart-Piltant in [16, Definition 2.15], and studied there in a more general context, including the arithmetic case. We also refer to the work of Kawanoue-Matsuki ([38], [39]) for other invariants attached to singularities in positive characteristic.

\section{Some Basics and an Overall View of the Paper}

2.1. Hironaka proved resolution of singularities in characteristic zero in $[28,29]$. His proof is existential, whereas we are going to discuss here constructive, or algorithmic, proofs of this theorem.

As a first approach, an algorithm of resolution of singularities would be a procedure in which once a variety is fixed, the result is a unique sequence of transformations, and the final transform of the variety is smooth:

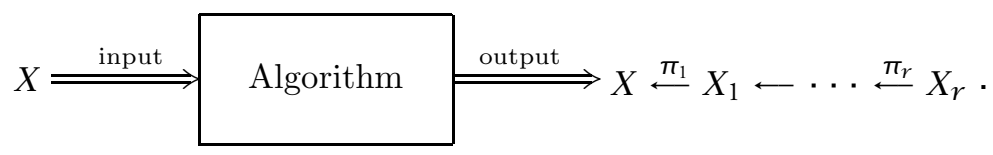

It is natural to require some properties for this algorithm, such as the following: 
- Compatibility with isomorphisms: Fix two schemes $X$ and $Y$ and assume that $\theta: X \rightarrow Y$ is an isomorphism of schemes. Once we fix a smooth subscheme $C \subset X$, we can consider the blow-up along $C$ (e.g., $X \stackrel{\pi_{C}}{{ }^{\prime}} X_{1}$ ). On the other hand, consider the blow-up along $\theta(C)$ (e.g., $Y \stackrel{\pi_{\theta(C)}}{\longleftarrow} Y_{1}$ ). This gives rise to a square diagram

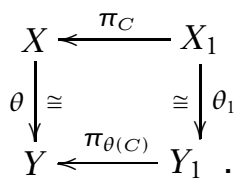

Having this in mind, it is quite predictable to require the algorithm of resolution to be compatible with isomorphisms, namely, that the sequences of transformations assigned by the algorithm to $Y$ and to $X$ should be related in this manner (by considering the fiber product).

- Compatibility with restriction to open subsets

- Compatibility with smooth morphisms.

The last two properties can be explained in the same way we have described the compatibility with isomorphisms.

These properties are known to hold for constructive resolutions ([46]). There are multiple forms to create an algorithm (and in fact, there are many of them).

The importance of the notion of compatibility with isomorphisms was indicated by Hironaka in [33]. However, this concept evolves from the theory of Trees, Groves, and Polygroves, developed by Hironaka in earlier publications (see, e.g., [32]). These ideas are the starting point for the concept of "invariant" as used by Hironaka. They play a relevant role in the globalization of local data (e.g., [30]). An invariant function will be a function defined in points of a variety that fulfills the natural properties of the previous discussion: namely,

- Two isomorphic varieties will have the same invariant function. In other words, if $X \stackrel{\theta}{\rightarrow} Y$ is an isomorphism of varieties, and $i_{X}, i_{Y}$ are the correspondent invariant functions, then $i_{X}(x)=i_{Y}(\theta(x))$.

- There will be good behavior with restriction to open sets and with étale topology.

A first example of an invariant function is the Hilbert-Samuel function that we will discuss below.

Remark 2.1. An algorithm of resolution of singularities is our first example of invariant function. Despite the fact that the Hilbert-Samuel function is an invariant function, it is not enough to prove resolution of singularities; however, it will be the first step in the search for an algorithm. In fact, algorithms of resolution are given as a suitable refinement of the Hilbert-Samuel function (see [8], [19], [45], [50]). 
2.2. Let $X \subset V^{(d)}$ be a hypersurface embedded in a $d$-dimensional smooth scheme over a perfect field $k$. An upper semi-continuous function, for instance,

$$
\text { mult }: X \rightarrow \mathbb{Z},
$$

is defined by setting mult $(x)$ as the multiplicity of $X$ at $x$.

If $b \in \mathbb{Z}$ denotes the highest value achieved by this function, then the set of $b$-fold points (e.g., $X(b)=\{x \in X \mid \operatorname{mult}(x)=b\}$ ) is closed. We encompass this situation in a more general setting that leads us to the notion of pairs, as follows. Pairs: Set, as before, $V^{(d)}$ a $d$-dimensional smooth scheme over a perfect field $k$. Fix a non-zero sheaf of ideals (e.g., $J \subset \mathcal{O}_{V^{(d)}}$ ), and a positive integer $b$. Here, $(J, b)$ is called a pair. It defines a closed subset in $V^{(d)}$, called the singular locus of $(J, b)$, for example,

$$
\operatorname{Sing}(J, b)=\left\{x \in V^{(d)} \mid v_{x}(J) \geq b\right\},
$$

where $v_{x}(J)$ denotes the order of $J$ in regular local ring $\mathcal{O}_{V^{(d)}, x}$. When $J=I(X)$ and $X$ is a hypersurface, the singular locus $\operatorname{Sing}(J, b)$ is the set of $b$-fold points of $X$.

Let $Y$ be a smooth irreducible subscheme included in $\operatorname{Sing}(J, b)$, and let $V^{(d)} \stackrel{\pi_{Y}}{=} V_{1}^{(d)}$ denote the monoidal transformation with center $Y$. Note that there is a factorization of the form

$$
J \mathcal{O}_{V_{1}^{(d)}}=I(H)^{b} J_{1},
$$

where $H=\pi_{Y}^{-1}(Y)$ denotes the exceptional hypersurface. This defines a new pair $\left(J_{1}, b\right)$, called the transform of $(J, b)$. Similarly, an iteration of transformations, for instance,

$$
\begin{aligned}
& (J, b) \quad\left(J_{1}, b\right) \\
& V^{(d)} \stackrel{\pi_{Y}}{\longleftarrow} V_{1}^{(d)} \stackrel{\pi_{Y_{1}}}{\longleftarrow} \cdots \stackrel{\pi_{Y_{r}-1}}{\longleftarrow} V_{r}^{(d)},
\end{aligned}
$$

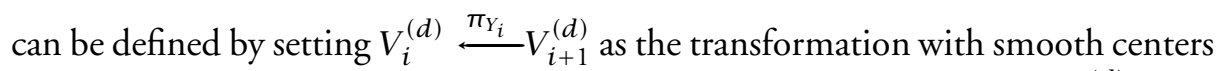
$Y_{i} \subset \operatorname{Sing}\left(J_{i}, b\right)$. Let $H_{i+1}$ denote the new exceptional hypersurface in $V_{i+1}^{(d)}$.

We shall always assume that such sequences are defined in such a way that the strict transforms of the $r$ exceptional hypersurfaces (e.g., $\left\{H_{1}, \ldots, H_{r}\right\}$ ) have normal crossings in $V_{r}^{(d)}$. A sequence of transformation, as above, is said to be a resolution if, in addition, $\operatorname{Sing}\left(J_{r}, b\right)=\varnothing$.

In the case of hypersurfaces, a resolution of the pair $(I(X), b)$ defines an elimination of the $b$-fold points of $X$ by blowing up centers included in the successive $b$-fold points of the strict transforms of the hypersurface.

Our strategy to define a refinement of the multiplicity will be accomplished in steps. A first approach is to search for functions defined on the class of pairs, 
namely, functions defined in some prescribed way along the closed sets defined by pairs.

For more general varieties $X \subset V^{(d)}$, one can work with the Hilbert-Samuel function. Consider, at each closed point $\xi \in X$, the graph of the Hilbert-Samuel function at the local ring $\mathcal{O}_{X, \xi}$. This is a function from $\mathbb{N}$ to $\mathbb{N}$, so the graph is an element of $\mathbb{N}^{\mathbb{N}}$, which we consider with the lexicographic ordering. It is possible to extend this function to non-closed points, for example,

$$
\mathrm{HS}_{X}: X \rightarrow \mathbb{N}^{\mathbb{N}} \text {, }
$$

in such a way that it is upper semicontinuous. The stratification on $X$ defined by this function is called the Hilbert-Samuel stratification (each stratum is called a Hilbert-Samuel stratum). Consider $\max \mathrm{HS}_{X}$ to be the maximum value of $\mathrm{HS}_{X}$, and denote by $\operatorname{Max}_{\mathrm{H}} \mathrm{HS}_{X}$ the maximum stratum of the Hilbert-Samuel function (e.g., $\operatorname{Max} \mathrm{HS}_{X}=\left\{\xi \in X \mid \mathrm{HS}_{X}(\xi)=\max \mathrm{HS}_{X}\right\}$ ). In the same way as with the multiplicity for a hypersurface discussed before, Hironaka defines a pair $(J, b)$ with the following properties:

(1) $\underline{\operatorname{Max}} \mathrm{HS}_{X}=\operatorname{Sing}(J, b)$.

(2) Any sequence of transformations of pairs

$$
\begin{aligned}
& V^{(d)} \stackrel{\pi_{Y}}{\longleftarrow} V_{1}^{(d)} \stackrel{\pi_{Y_{1}}}{\longleftarrow} \cdots \stackrel{\pi_{Y_{r-1}}}{\longleftarrow} V_{r}^{(d)} \\
& (J, b) \quad\left(J_{1}, b\right) \quad\left(J_{r}, b\right)
\end{aligned}
$$

will induce a sequence of transformations over $X$, for instance,

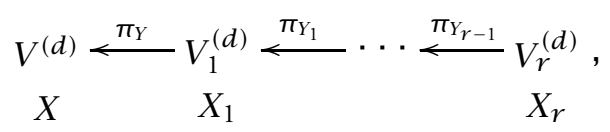

and moreover, the equality

$$
\underline{\operatorname{Max}} \mathrm{HS}_{X_{i}}=\operatorname{Sing}\left(J_{i}, b\right)
$$

holds for any index $i<r$.

Note that if (2.2) is a resolution of the pair $(J, b)$, namely, if $\operatorname{Sing}\left(J_{r}, b\right)=\varnothing$, then the maximum value of the Hilbert-Samuel function drops at $X_{r}$ for the induced sequence (2.3). Thus, resolution of pairs leads to improvement of the maximum value of $\mathrm{HS}_{X}$; moreover, Hironaka proves that resolution of singularities is achieved by iterating this procedure.

Problem. We have fixed $X \subset V^{(d)}$ and attached to it a pair $(J, b)$. Assume now that one considers a different embedding of $X$ (e.g., $\left.X \subset V^{\prime(d)}\right)$ with the same dimension $d$. Suppose there is a new pair $\left(J^{\prime}, b^{\prime}\right)$ that fulfills the two previous properties. It is natural to require that the induced transformations defined over $X$, defined by a resolution of $(J, b)$, be the same as that obtained by a resolution of $\left(J^{\prime}, b^{\prime}\right)$. 
In order to address these questions, we define an algorithm of resolution of basic objects. Namely, we define a totally ordered set $\Gamma$, and, for each pair $(J, b)$, an upper semi-continuous function $\delta_{(J, b)}: \operatorname{Sing}(J, b) \rightarrow \Gamma$, so that the closed stratum corresponding to the biggest value (e.g., $\left.\underline{\operatorname{Max}} \delta_{(J, b)}\right)$ is regular, and such that a resolution of the pair is achieved by successive blow-ups at such stratum.

Requirement. The requirement we impose on an algorithm is now clear: given $X \subset V^{(d)}$ and a pair $(J, b)$, and given $X \subset V^{\prime(d)}$ and a pair $\left(J^{\prime}, b^{\prime}\right)$, both in the previous conditions for the same $X$, we require that the sequence of transformations defined over $X$ by the resolution of $(J, b)$ be the same as that defined by the resolution of $\left(J^{\prime}, b^{\prime}\right)$.

Note here that (2.4) allows us to define these functions on

$$
\underline{\operatorname{Max}} \mathrm{HS}_{X_{i}}\left(=\operatorname{Sing}\left(J_{i}, b\right)=\operatorname{Sing}\left(J_{i}^{\prime}, b^{\prime}\right)\right) .
$$

The requirement is fulfilled if the induced functions over $X$ are independent of the pairs $(J, b)$ and $\left(J^{\prime} b^{\prime}\right)$ described before, and if this fact is preserved by the prescribed blow-ups.

A first step in the definition of this algorithm, subject to this requirement, is given by Hironaka's function.

Remark 2.2. Hironaka defines a function $\operatorname{ord}_{(J, b)}: \operatorname{Sing}(J, b) \longrightarrow \mathbb{Q}$ by

$$
\operatorname{ord}_{(J, b)}(\xi)=\frac{\nu_{\xi}(J)}{b}
$$

where $v_{\xi}(J)$ is the order of ideal $J$ in the regular local ring $\mathcal{O}_{V^{(d)}, \xi}$. Recall that $\operatorname{Sing}(J, b)=\underline{\operatorname{Max}} H S_{X}=\operatorname{Sing}\left(J^{\prime}, b^{\prime}\right)$.

A remarkable property of this function, to be discussed below, is that

$$
\operatorname{ord}_{(J, b)}(\xi)=\operatorname{ord}_{\left(J^{\prime}, b^{\prime}\right)}(\xi)
$$

for any $\xi \in \underline{\operatorname{Max}} \mathrm{HS}_{X}$. This fact leads to the definition of the function

$$
\operatorname{ord}_{X}^{(d)}: \underline{\operatorname{Max}} \mathrm{HS}_{X} \rightarrow \mathbb{Q}
$$

This key observation ensures that if the algorithm, namely $\Gamma$ and the functions $\delta_{(J, b)}: \operatorname{Sing}(J, b) \longrightarrow \Gamma$, are defined in terms of Hironaka's function, then the requirement is automatically fulfilled.

This is precisely how algorithms of resolution in characteristic zero are defined.

\subsection{On the equality (2.5) and the property of equivariance.}

Definition 2.3. Fix a point $x \in \operatorname{Sing}(J, b)$. A marked sequence over $x$ is given by the following: 
(1) A sequence of transformations

$$
\begin{aligned}
& V^{(d)} \stackrel{\pi_{1}}{\stackrel{n_{1}}{(d)}} \underset{(J, b)}{\stackrel{\pi_{2}}{\longleftarrow}} \cdots \underset{\left(J_{1}, b\right)}{\stackrel{\pi_{\ell-1}}{V_{\ell}}} V_{\ell}^{(d)} \\
& \left(J_{\ell}, b\right)
\end{aligned}
$$

where the center of the transformation $\pi_{j}$ is included in $\operatorname{Sing}\left(J_{j-1}, b\right)$;

(2) A collection of points $x_{0}, \ldots, x_{\ell}$, where

$$
\pi_{i}\left(x_{i}\right)=x_{i-1} \text { and } x_{0}=x .
$$

To be precise, in the previous sequence of transformations, one can intercalate other morphisms like restrictions to open sets or multiplications by affine spaces. We refer to [5] for more details on this notion.

Remark 2.4 (Theoretical observation on Hironaka's set). From the marked sequences over $\xi$, one can read the rational number $\operatorname{ord}_{(J, b)}(\xi)$. More precisely, this is given by $\operatorname{codim}_{x_{i}}\left(\operatorname{Sing}\left(J_{i}, b\right)\right)$ in $V_{i}^{(d)}$.

\section{Some consequences.}

- Note here that any marked sequence over $\xi$ of the form (2.6) induces a sequence of embedded transformations over $X$, for instance,

$$
\begin{aligned}
& V^{(d)} \stackrel{\pi_{1}}{\longleftarrow} V_{1}^{(d)} \stackrel{\pi_{2}}{\longleftarrow} \cdots \stackrel{\pi_{\ell-1}}{\longleftarrow} V_{\ell}^{(d)} \\
& \begin{array}{lll}
X & X_{1} & X_{\ell},
\end{array}
\end{aligned}
$$

and a collection of points $x_{i} \in X_{i}$, where $\pi_{i}\left(x_{i}\right)=x_{i-1}$ and $x_{0}=\xi$.

The previous observation can be reformulated in such a way that the rational number $\operatorname{ord}_{(J, b)}(\xi)$ is given by $\operatorname{codim}_{x_{i}}\left(\underline{\operatorname{Max}} \mathrm{HS}_{X_{i}}\right)$ in $V_{i}^{(d)}$. In particular, we then have $\operatorname{ord}_{(J, b)}(\xi)=\operatorname{ord}_{\left(J^{\prime} b^{\prime}\right)}(\xi)$ via the identifications $\operatorname{Sing}\left(J_{i}, b\right)=\underline{\operatorname{Max}} \mathrm{HS}_{X_{i}}=\operatorname{Sing}\left(J_{i}^{\prime}, b^{\prime}\right)$.

- Let $\alpha: X \longrightarrow Y$ be an isomorphism of schemes. We had the data $X \subset V^{(d)}$, and the pair $(J, b)$. The isomorphism $\alpha$ provides an inclusion $Y \subset V^{(d)}$, and the same pair $(J, b)$ is a representative of $\underline{\mathrm{Max}} \mathrm{HS}_{Y}$. The previous observation implies that $\operatorname{ord}_{Y}(\alpha(\xi))=\operatorname{ord}_{X}(\xi)$.

- As we said before, any algorithm of resolution of a basic object using this order function will inherit the property of equivariance.

- The functions or invariant functions described in this paper are defined in a way analogous to that of Hironaka's inductive functions, so they inherit all the good properties described before.

2.4. The order function, and lower-dimensional H-functions. An example of a function on the class of pairs is Hironaka's $\tau$-function. For each pair $(J, b)$, over $V^{(d)}$, a lower semi-continuous function

$$
\tau_{(J, b)}: \operatorname{Sing}(J, b) \longrightarrow \mathbb{Z}_{\geq 0}
$$


is defined (or, say, $(-1) \cdot \tau_{(J, b)}$ is upper semi-continuous). The value we have at $x \in \operatorname{Sing}(J, b)$ (e.g., $\left.\tau_{(J, b)}(x)\right)$ is an integer encoding fundamental information.

When $x$ is a closed point in $V^{(d)}$, then $g r_{M}\left(\mathcal{O}_{V^{(d)}, x}\right)$ is a polynomial ring over a perfect field, and $\tau_{(J, b)}(x)$ is defined as the least number of variables required to express the homogenous ideal spanned by $\left(J+M^{b+1}\right) / M^{b+1}$.

In practical terms, and for the sake of resolution, the invariant $\tau_{(J, b)}(x)$ indicates the number of variables that can be eliminated. This claim will be clarified below, but let us indicate some properties that support it.

In the case of characteristic zero, given that $x \in \operatorname{Sing}(J, b)$, and if we set $e=\tau_{(J, b)}(x)$, there is then a smooth subscheme of dimension $d-e$ inside $V^{(d)}$ (e.g., $\left.V^{(d-e)} \subset V^{(d)}\right)$, together with a pair $\left(\bar{J}, b^{\prime}\right)$, with the property that a resolution of $\left(\bar{J}, b^{\prime}\right)$ defines a resolution of $(J, b)$.

This construction can be done in a neighborhood of $x$. The advantage of this reformulation is that $\bar{J}$ is an ideal in $V^{(d-e)}$, and hence involves $e$ variables less. The underlying idea is, of course, that the smaller the dimension of $V^{(d-e)}$ is, the easier it is to construct a resolution. Here, $V^{(d-e)}$ is defined so that, locally, $\operatorname{Sing}(J, b) \subset V^{(d-e)}$, and moreover, $\operatorname{Sing}(J, b)=\operatorname{Sing}\left(\bar{J}, b^{\prime}\right)$.

Example 2.5. In the case of $f=y^{2}+z^{3}+x^{7} \in k[x, y, z]$, the $\tau$ invariant of the pair $(\langle f\rangle, 2)$ is in this case equal to 1 at the origin, and $y$ is the variable that can be eliminated. Here, we have that $V^{(d-e)}=V^{(2)}=\operatorname{Spec}(k[x, z])$, and $\left(\bar{J}, b^{\prime}=\left(\left\langle z^{3}+x^{7}\right\rangle, 2\right)\right.$.

We now introduce one of the main objects of interest in this work, the socalled $r$-dimensional H-functions

$$
\mathrm{H}-\operatorname{ord}_{(J, b)}^{(r)}: \operatorname{Sing}(J, b) \rightarrow \mathbb{Q} .
$$

These functions are somehow subordinated to the previous $\tau$-function. In fact, if $e=\tau_{(J, b)}(x)$, then at least $e$ variables can be eliminated. In this case, we shall define functions $\mathrm{H}-\operatorname{ord}_{(J, b)}^{(r)}$ at a suitable neighborhood of $x$, but only for $d-e \leq r \leq d$.

For the case $r=d$, the function $\mathrm{H}$-ord $(J, b)$ is always defined. In this case, $\mathrm{H}-\operatorname{ord}_{(J, b)}^{(d)}=\operatorname{ord}_{(J, b)}^{(d)}$, where

$$
\operatorname{ord}_{(J, b)}^{(d)}: \operatorname{Sing}(J, b) \longrightarrow \mathbb{Q} \quad \text { with } \operatorname{ord}_{(J, b)}^{(d)}(y)=\frac{v_{y}(J)}{b}
$$

for $y \in \operatorname{Sing}(J, b)$, and $v_{y}(J)$ denotes the order of $J$ in $\mathcal{O}_{V^{(d)}, y}$. Thus, the $d$-dimensional function coincides with Hironaka's well-known order function, namely, $\operatorname{ord}_{(J, b)}$. One checks easily that $\operatorname{ord}_{(J, b)}$ is upper semi-continuous.

A property of these functions $\mathrm{H}-\operatorname{ord}_{(J, b)}^{(r)}$ is that they are constantly equal to 1 at points of $\operatorname{Sing}(J, b)$, in a neighborhood of $x$, except for

$$
\mathrm{H}-\operatorname{ord}_{(J, b)}^{(d-e)}: \operatorname{Sing}(J, b) \longrightarrow \mathbb{Q} .
$$


When the characteristic is zero, H-ord ${ }^{(d-e)}$ admits the following description: set $V^{(d-e)} \subset V^{(d)}$ and $\left(\bar{J}, b^{\prime}\right)$, as above. Recall that $\operatorname{Sing}(J, b)=\operatorname{Sing}\left(\bar{J}, b^{\prime}\right)$, and set

$$
\mathrm{H}-\operatorname{ord}_{(J, b)}^{(d-e)}(y)=\frac{v_{y}(\bar{J})}{b^{\prime}},
$$

for $y \in \operatorname{Sing}(J, b)$, where $v_{y}(\bar{J})$ denotes the order of $\bar{J}$ at $\mathcal{O}_{V^{(d-e)}, y}$. This local description, which holds only in characteristic zero, indicates that (2.7) is upper semi-continuous.

The previous definition makes use of the existence of hypersurfaces of maximal contact, which is a particular feature of characteristic zero. In the case of positive characteristic, $\mathrm{H}-\operatorname{ord}_{(J, b)}^{(d-e)}(y)$ does not admit a local description as in (2.8). Moreover, it will be shown that function (2.7) is no longer upper semi-continuous (see Example 2.9).

2.5. Objective of this work. We shall indicate later how $\mathrm{H}-\operatorname{ord}_{(J, b)}^{(d-e)}(y)$ is defined over fields of positive characteristic. The behavior of this function is rather untraceable. It has been studied in previous works (e.g., [42], [43] [11], [14], [15], [26]), but only as a refinement of hypersurface singularities. Our results here are directed towards singularities of arbitrary schemes over perfect fields, and the outcome is a refinement of the Hilbert-Samuel stratification.

Set $r=d-e$. As previously indicated, in general the functions $\mathrm{H}-\operatorname{ord}_{(J, b)}^{(r)}$ : $\operatorname{Sing}(J, b) \longrightarrow \mathbb{Q}$ are not upper semi-continuous; one cannot expect a nice inductive formulation, as that in (2.8), which is valid only in characteristic zero. In this paper, we show that this lack of semicontinuity can be somehow bounded; to this end, we present two functions on pairs: the roof function, for instance,

$$
R_{(J, b)}^{(r)}: \operatorname{Sing}(J, b) \longrightarrow \mathbb{Q}
$$

and the floor function, for instance,

$$
F_{(J, b)}^{(r)}: \operatorname{Sing}(J, b) \rightarrow \mathbb{Q}
$$

Both $R_{(J, b)}^{(r)}$ and $F_{(J, b)}^{(r)}$ are upper semi-continuous functions, and

$$
F_{(J, b)}^{r} \leq \mathrm{H}-\operatorname{ord}_{(J, b)}^{(r)} \leq R_{(J, b)}^{(r)} .
$$

In characteristic zero we get an equality on the right-hand side. In particular, $\operatorname{ord}_{(J, b)}^{(r)}$ is upper semi-continuous.

The objective of this paper is to explore these functions in positive characteristic, and to show that resolution of pairs $(J, b)$ is attained when one of the inequalities is an equality. 
The roof functions $R^{(r)}$ will be discussed in 2.12, and the floor functions $F^{(r)}$ in 2.14. Some indications on the behavior of the $\mathrm{H}$-functions $\mathrm{H}$-ord $(J, b)$ will be discussed in 2.15 .

A precise formulation of the previous statement is given in Theorem 2.13. A first step in this direction requires a harmless reformulation, in which pairs are replaced by Rees algebras (see 2.6). The advantage of this reformulation is that each algebra can be naturally enlarged to a new algebra that is enriched by the action of differential operators. These are called differential Rees algebras, which have been recently studied, because of the strong properties they have, by several authors such as Hironaka, Kawanoue-Matsuki, and Włodarczyk (e.g., [36], [38], [39], [47], [48], [9], [6], [51]). Moreover, for the purpose of our study, we can always restrict attention to these algebras compatible with differential operators.

2.6. From pairs to Rees algebras. A pair over $V^{(d)}$ can be viewed as an algebra. A Rees algebra over $V^{(d)}$ is an algebra of the form $G=\bigoplus_{n \in \mathbb{N}} I_{n} W^{n}$, where $I_{0}=\mathcal{O}_{V^{(d)}}$ and each $I_{n}$ is a coherent sheaf of ideals. Here, $W$ denotes a dummy variable introduced to keep track of the degree, so $G \subset \mathcal{O}_{V^{(d)}}[W]$ is an inclusion of graded algebras. It is always assumed that, locally at any point of $V^{(d)}$, $G$ is a finitely generated $\mathcal{O}_{V^{(d)}}$-algebra. Specifically, when one restricts to an affine set, there are local generators (e.g., $\left\{f_{n_{1}}, \ldots, f_{n_{s}}\right\}$ ), so that

$$
\mathcal{G}=\mathcal{O}_{V^{(d)}}\left[f_{n_{1}} W^{n_{1}}, \ldots, f_{n_{s}} W^{n_{s}}\right] \quad\left(\subset \mathcal{O}_{V^{(d)}}[W]\right) .
$$

We now define the singular locus of $\mathcal{G}=\oplus I_{n} W^{n}$ to be the closed set

$$
\operatorname{Sing}(G):=\left\{x \in V^{(d)} \mid v_{x}\left(I_{n}\right) \geq n \text { for each } n \in \mathbb{N}\right\} .
$$

Fix a monoidal transformation $V^{(d)} \stackrel{\pi_{C}}{\stackrel{(d)}{-}} V_{1}^{(d)}$ with center $C \subset \operatorname{Sing}(G)$. For all $n \in \mathbb{N}$, there is a factorization of the form

$$
I_{n} \mathcal{O}_{V_{1}^{(d)}}=I\left(H_{1}\right)^{n} \cdot I_{n}^{(1)},
$$

where $H_{1}=\pi_{C}^{-1}(C)$ denotes the exceptional hypersurface. This defines a Rees algebra over $V_{1}^{(d)}$ (e.g., $G_{1}=\bigoplus_{n \in \mathbb{N}} I_{n}^{(1)} W^{n}$ ), called the transform of $G$. This transformation will be denoted by

$$
\begin{array}{cr}
\mathcal{G} & \mathcal{G}_{1} . \\
V^{(d)} \stackrel{\pi_{C}}{\longleftarrow} V_{1}^{(d)}
\end{array}
$$

A sequence of transformations will be denoted by

$$
\begin{array}{cccc}
\mathcal{G} & G_{1} & G_{r} \\
V^{(d)} \stackrel{\pi_{C}}{\longleftarrow} V_{1}^{(d)} \stackrel{\pi_{C_{1}}}{\longleftarrow} \cdots \stackrel{\pi_{C_{r-1}}}{\longleftarrow} V_{r}^{(d)},
\end{array}
$$


and herein we always assume that the exceptional locus of the composite morphism $V^{(d)} \leftarrow V_{r}^{(d)}$ (e.g., $\left\{H_{1}, \ldots, H_{r}\right\}$ ) is a union of hypersurfaces with only normal crossings in $V_{r}^{(d)}$. A sequence (2.12) is said to be a resolution of $G$ if, in addition, $\operatorname{Sing}\left(G_{r}\right)=\varnothing$.

A pair $(J, b)$ over $V^{(d)}$ defines an algebra, for instance,

$$
G_{(J, b)}=\mathcal{O}_{V^{(d)}}\left[J W^{b}\right] .
$$

Note here that $\operatorname{Sing}(J, b)=\operatorname{Sing}\left(\mathcal{G}_{(J, b)}\right)$; moreover, if we consider $\mathcal{G}=\mathcal{G}_{(J, b)}$ in (2.11), then $G_{1}=G_{\left(J_{1}, b\right)}$, where $\left(J_{1}, b\right)$ denotes the transform of $(J, b)$. Thus, algebras appear as a naive reformulation of pairs, and a resolution of $\mathcal{G}_{(J, b)}$ is the same as a resolution of $(J, b)$. Furthermore, all the previous discussion, developed in terms of pairs, has a natural analog for Rees algebras. We first discuss, briefly, the formulation of the $\tau$-invariant for algebras over $V^{(d)}$.

2.7. Hironaka's $T$-invariant. Fix a Rees algebra $G$ over $V^{(d)}$ and a closed point $x \in \operatorname{Sing}(G)$. Recall that the tangent space at $x$ is

$$
\mathbb{T}_{V^{(d)}, x}=\operatorname{Spec}\left(g r_{M}\left(\mathcal{O}_{V^{(d)}, x}\right)\right) .
$$

An homogeneous ideal is attached to $G$ at the closed point $x$ (e.g., $\operatorname{In}_{x}(G)$ ), is included in $g r_{\mathfrak{M}_{x}}\left(\mathcal{O}_{V^{(d)}, x}\right)$, and is defined as homogeneous ideal generated by the class of $I_{n}$ at the quotient $\mathfrak{M}_{x}^{n} / \mathfrak{M}_{x}^{n+1}$, for all $n$. The tangent cone at $x$ (e.g., $C_{G}(x) \subset \mathbb{T}_{V^{(d)}, x}$ ) will be the cone defined by this homogeneous ideal. There is a largest subspace, denoted by $\mathcal{L}_{\mathcal{G}}$, which is included and acting by translations on $C_{G}$. This linear space is called the space of vertices of $C_{G}(x)$. Finally, the $T$-invariant at a closed point is defined by setting $\tau_{\mathcal{G}}(x)\left(=\tau_{\mathcal{G}, x}\right)$ to be the codimension of $\mathcal{L}_{G, x}$ in $\mathbb{T}_{V^{(d)}, x}$. Here, we are going to consider the $\tau$-invariant only at closed points $x$. In this setting, whenever $x$ is closed, $\tau_{\mathcal{G}}(x)$ is the fewest number of variables required to express generators of the ideal $\operatorname{In}_{x}(G)$ in the polynomial ring $g r_{M}\left(\mathcal{O}_{V^{(d)}, x}\right)$.

For the particular case of $G=G_{(J, b)}$, we thus get $\tau_{\mathcal{G}}(x)=\tau_{(J, b)}(x)$ at any $x \in \operatorname{Sing}(J, b)=\operatorname{Sing}(G)$.

2.8. Rees algebras and differential structure. There are various advantages in formulating invariants in terms of Rees algebras as opposed to formulating them in terms of pairs. One of these advantages arises when one studies Rees algebras with a natural compatibility with differential operators.

A Rees algebra $G=\bigoplus_{n \geq 0} I_{n} W^{n}$ over $V^{(d)}$ is said to be a differential Rees algebra if, locally (e.g., over any open affine set) $\mathrm{D}_{r}\left(I_{n}\right) \subset I_{n-r}$, for any index $n$ and for any differential operator $\mathrm{D}_{r}$ of order $r<n$.

If this property holds for all $k$-linear differential operators, then we say that $G$ is an absolute differential Rees algebra over the smooth scheme $V^{(d)}$. It is convenient to extend the previous definition to the relative context, for example, when a 
smooth morphism $V^{(d)} \stackrel{\beta}{\longrightarrow} V^{\left(d^{\prime}\right)}$ is fixed. If the previous property holds for differential operators which are $\mathcal{O}_{V^{\left(d^{\prime}\right)}}$-linear, or, for instance, $\beta$-relative operators, then $G$ is said to be a $\beta$-relative differential Rees algebra, or simply $\beta$-differential.

Proposition 2.6 ([47, Theorems 3.2 and 4.1]). Every Rees algebra $G$ over $V^{(d)}$ admits an extension to a new Rees algebra (e.g., $G \subset \operatorname{Diff}(\mathcal{G})$ ), so that $\operatorname{Diff}(\mathcal{G})$ is a differential Rees algebra. It has the following properties:

(1) $\operatorname{Diff}(G)$ is the smallest differential Rees algebra containing $G$.

(2) $\operatorname{Sing}(G)=\operatorname{Sing}(\operatorname{Diff}(G))$.

(3) The equality in (2) is preserved by transformations. In particular, any resolution of $G$ defines a resolution of $\operatorname{Diff}(G)$, and the converse holds.

The property in (3) says that, for the sake of defining a resolution of $G$, we may always assume that it is a differential Rees algebra. This is an important reduction because, as we shall see, differential Rees algebras have very powerful properties. Further details can be found in [47].

2.9. Transversal projections and elimination. Once we fix a closed point $x \in V^{(d)}$, it is very simple to construct, for any positive integer $d^{\prime} \leq d$, a smooth scheme $V^{\left(d^{\prime}\right)}$ together with a smooth morphism $\beta: V^{(d)} \longrightarrow V^{\left(d^{\prime}\right)}$ (a projection), at least when we restrict $V^{(d)}$ to an étale neighborhood of $x$. The claim follows, essentially, from the definition of smoothness. In fact, $\left(V^{(d)}, x\right)$ is an étale neighborhood of $\left(\mathbb{A}^{(d)}, \mathbb{O}\right)$, and plenty of smooth morphisms (i.e., plenty of surjective linear transformations) $\left(\mathbb{A}^{(d)}, \mathbb{O}\right) \longrightarrow\left(\mathbb{A}^{\left(d^{\prime}\right)}, \mathbb{O}\right)$ can be constructed. Note that if $\left\{x_{1}, \ldots, x_{d}\right\}$ is a regular system of parameters at $\mathcal{O}_{V^{(d)}, x}$, then $\left(V^{(d)}, x\right)$ is an étale neighborhood of $\mathbb{A}_{k}^{(d)}=\operatorname{Spec}\left(k\left[x_{1}, \ldots, x_{d}\right]\right)$ at the origin.

Furthermore, given a subspace $S$ of dimension $d-d^{\prime}$ in $\mathbb{T}_{V^{(d)}, x}$, one can easily construct $V^{\left(d^{\prime}\right)}$ and a smooth $\beta: V^{(d)} \longrightarrow V^{\left(d^{\prime}\right)}$ so that $\operatorname{ker}\left(d(\beta)_{x}\right)=S$ (here, $d(\beta)_{x}: \mathbb{V}_{V^{(d)}, x} \rightarrow \mathbb{T}_{V^{\left(d^{\prime}\right)}, \beta(x)}$ is a surjective linear transformation).

Fix now a differential Rees algebra over $V^{(d)}$ and a closed point $x \in \operatorname{Sing}(G)$. Recall here that $\tau_{\mathcal{G}}(x)=e$ is the codimension of $\mathcal{L}_{\mathcal{G}, x}\left(\subset \mathcal{C}_{G, x}\right)$ in the tangent space. Set $d^{\prime}$ so that $d \geq d^{\prime} \geq d-e$.

For $d^{\prime}$ in these conditions, we say that a smooth morphism $\beta: V^{(d)} \longrightarrow V^{\left(d^{\prime}\right)}$ is transversal to $G$ at $x$ if

$$
\operatorname{ker}(d \beta)_{x} \cap \mathcal{L}_{G, x}=\mathbb{O} .
$$

This condition is open (it holds at points in a neighborhood of $x$ ), and a smooth morphism $\beta: V^{(d)} \longrightarrow V^{\left(d^{\prime}\right)}$ is said to be transversal to $\mathcal{G}$, if this condition holds at any point of $\operatorname{Sing}(\mathcal{G})$.

Since $G$ is a differential Rees algebra, it is, in particular, a $\beta$-differential Rees algebra. 
Proposition 2.7. Assume that $\beta: V^{(d)} \longrightarrow V^{\left(d^{\prime}\right)}$ is transversal to $G$, and that $G$ is $\beta$-differential. Then, a Rees algebra $\mathcal{R}_{G, \beta}$ is defined over $V^{\left(d^{\prime}\right)}$, for instance,

$$
\begin{array}{crr}
\mathcal{G} & \mathcal{R}_{\mathcal{G}, \beta} \\
V^{(d)} \stackrel{\beta}{\longrightarrow} & V^{\left(d^{\prime}\right)}
\end{array}
$$

(i.e., $\left.\mathcal{R}_{G, \beta} \subset \mathcal{O}_{V^{\left(d^{\prime}\right)}}[W]\right)$, with the following properties:

(1) The natural lifting of $\mathcal{R}_{G, \beta}\left(\right.$ e.g., $\left.\beta^{*}\left(\mathcal{R}_{\mathcal{G}, \beta}\right)\right)$ is a subalgebra of $\mathcal{G}$ ([48, Theorem 4.13]).

(2) $\beta(\operatorname{Sing}(\mathcal{G})) \subset \operatorname{Sing}\left(\mathcal{R}_{\mathcal{G}, \beta}\right)$; moreover, $\left.\beta\right|_{\operatorname{Sing}(\mathcal{G})}: \operatorname{Sing}(\mathcal{G}) \longrightarrow \operatorname{Sing}\left(\mathcal{R}_{\mathcal{G}, \beta}\right)$ defines a set theoretical bijection of $\operatorname{Sing}(G)$ with its image ([48, 1.15 and Theorem 4.11], or [9, 7.1]).

(3) Given a smooth sub-scheme $Y \subset \operatorname{Sing}(\mathcal{G}), \beta(Y)\left(\subset \operatorname{Sing}\left(\mathcal{R}_{\mathcal{G}, \beta}\right)\right)$ is isomorphic to $Y$. In particular, $Y$ defines a transformation of $\mathcal{G}$ and also of $\mathcal{R}_{\mathcal{G}, \beta}$ ([9, Theorem 9.1 (i)]).

(4) A smooth center $Y \subset \operatorname{Sing}(G)$ defines a commutative diagram

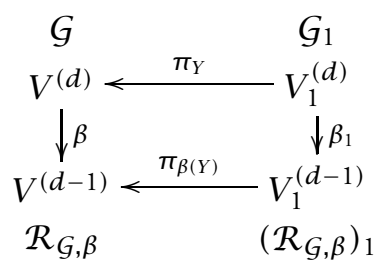

where $\mathcal{G}_{1}$ and $\left(\mathcal{R}_{\mathcal{G}, \beta}\right)_{1}$ denote the transforms of $\mathcal{G}$ and $\mathcal{R}_{\mathcal{G}, \beta}$, respectively ([9, Theorems 10.1 and 9.1]). Here, $\beta_{1}$ is defined in the restriction of $V_{1}^{(d)}$ to a neighborhood of $\operatorname{Sing}\left(G_{1}\right)$, and this diagram has the following properties:

(4a) $V_{1}^{(d)} \stackrel{\beta_{1}}{\longrightarrow} V_{1}^{\left(d^{\prime}\right)}$ is transversal to $G_{1}$ and $G_{1}$ is $\beta_{1}$-differential. In particular, we get

$$
\begin{array}{cr}
G_{1} \quad \mathcal{R}_{\mathcal{G}_{1}, \beta_{1}} . \\
V_{1}^{(d)} \stackrel{\beta_{1}}{\longrightarrow} V_{1}^{\left(d^{\prime}\right)}
\end{array}
$$

(4b) $\left(\mathcal{R}_{G, \beta}\right)_{1}$ coincides with the algebra $\mathcal{R}_{\mathcal{G}_{1}, \beta_{1}}$, defined by $\beta_{1}$.

The algebra $\mathcal{R}_{\mathcal{G}, \beta}$ over $V^{\left(d^{\prime}\right)}$ defined by a transversal $\beta: V^{(d)} \longrightarrow V^{\left(d^{\prime}\right)}$ as in (2.13) is called the elimination algebra of $\mathcal{G}$ defined by $\beta$.

2.10. The previous proposition says that, given $\beta: V^{(d)} \longrightarrow V^{\left(d^{\prime}\right)}$ transversal to $G$, and if $\mathcal{G}$ is a $\beta$-differential Rees algebra (e.g., if $G$ is an absolute differential Rees algebra), then an arbitrary sequence of monoidal transformations, for instance,

$$
\begin{array}{cccc}
\mathcal{G} & G_{1} & G_{r} \\
V^{(d)} \stackrel{\pi_{Y}}{\longleftarrow} V_{1}^{(d)} \stackrel{\pi_{Y_{1}}}{\longleftarrow} \cdots \stackrel{{ }^{\pi_{Y_{r-1}}}}{\longleftarrow} V_{r}^{(d)},
\end{array}
$$


gives rise to a diagram, for instance,

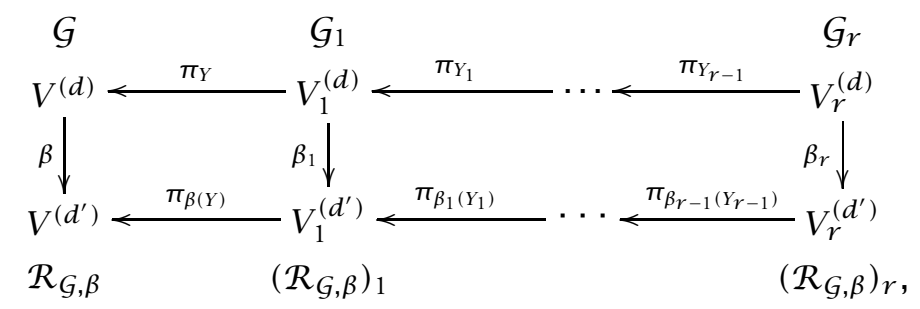

where the following hold:

(1) For any index $i$, there is an inclusion $\left(\mathcal{R}_{\mathcal{G}, \beta}\right)_{i} \subset \mathcal{G}_{i}$.

(2) Every $\beta_{i}$ is transversal to $G_{i}$, and $G_{i}$ is $\beta_{i}$-differential.

(3) $V_{i}^{\left(d^{\prime}\right)} \stackrel{\pi_{\beta\left(Y_{i}\right)}}{\left(d^{\prime}\right)} V_{i+1}$ denotes the transformation with center $\beta_{i}\left(Y_{i}\right)$ that is isomorphic to $Y_{i}$.

(4) $\left(\mathcal{R}_{\mathcal{G}, \beta}\right)_{i}=\mathcal{R}_{\mathcal{G}_{i}, \beta_{i}}$ where the latter denotes the elimination algebra of $\mathcal{G}_{i}$ with respect to $\beta_{i}$.

(5) $\beta_{i}\left(\operatorname{Sing}\left(G_{i}\right)\right) \subset \operatorname{Sing}\left(\left(\mathcal{R}_{\mathcal{G}, \beta}\right)_{i}\right)$, and $\left.\beta_{i}\right|_{\operatorname{Sing}\left(\mathcal{G}_{i}\right)}: \operatorname{Sing}\left(\mathcal{G}_{i}\right) \longrightarrow \beta_{i}\left(\operatorname{Sing}\left(G_{i}\right)\right)$ is an identification. In the characteristic zero case, the previous inclusions are equalities, but in positive characteristic, in general, only the inclusion holds.

Remark 2.8. It is convenient to consider the Rees algebra $G$ to be differential in the formulation of 2.10. We know this can be done since $G$ and its differential closure lead to the same resolution (see Proposition 2.6). In fact, in this case, locally at any closed point $x \in \operatorname{Sing}(G)$, one can construct a smooth scheme $V^{\left(d^{\prime}\right)}$ and a smooth morphism $\beta: V^{(d)} \longrightarrow V^{\left(d^{\prime}\right)}$, and then $G$ will always be a $\beta$-differential Rees algebra. In fact, an absolute differential Rees algebra is always relative differential for any smooth morphism $\beta: V^{(d)} \longrightarrow V^{\left(d^{\prime}\right)}$.

2.11. Order and lower-dimensional H-functions and Rees algebras. Fix a Rees algebra $G=\bigoplus I_{n} W^{n}$ over a $d$-dimensional smooth scheme $V^{(d)}$. Lower dimensional $\mathrm{H}$-functions

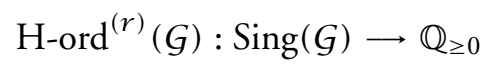

will be defined for $r$ in a certain range $d^{\prime} \leq r \leq d$ (for some $d^{\prime} \leq d$ ). For $r=d$, the function $\mathrm{H}$-ord ${ }^{(d)}$ is usually denoted simply by "ord"; we will use this notation in this work. In this case,

$$
\operatorname{ord}(G)(x)=\min \left\{\frac{v_{x}\left(I_{n}\right)}{n} \mid n \in \mathbb{N}\right\},
$$

with $x \in \operatorname{Sing}(G)$. 


\section{Facts.}

- If $\boldsymbol{T}_{\mathcal{G}}(x) \geq 1$, then the function is constantly equal to 1 in an open neighborhood of $x$.

- If $\tau_{\mathcal{G}}(x) \geq e$, then, in a neighborhood of $x$, the functions are defined in a range $d-e \leq r \leq d$. Moreover, $\mathrm{H}$-ord ${ }^{(r)}(G)$ is equal to 1 in a neighborhood of $x$ for $r \geq d-e+1$.

- A particular feature is that, in general, the function ord $(G)$ is upper semicontinuous.

- In contrast, if $\tau_{\mathcal{G}}(x) \geq e>0$, the function H-ord ${ }^{(d-e)}(G)$ might not be upper semi-continuous. The following example illustrates this fact.

Example 2.9. Fix $f=z^{p}+x y^{p} \in k[x, y, z]$ with $k$ a perfect field of characteristic $p>0$. In this example, the $\mathrm{H}$-function will be exactly $(p+1) / p$ in any closed point of the form $(x, y, z)=(\lambda, 0,0)$. Nevertheless, the value of the $\mathrm{H}$-function at the generic point of the line $y=z=0$ will be 1 .

2.12. On the upper-bound function. Fix a differential Rees algebra $G$. Let $e$ be a positive integer, and assume that $\tau_{\mathcal{G}}(x) \geq e$ at any closed point $x \in \operatorname{Sing}(G)$, so that the function $\operatorname{Ord}^{(d-e)}(G): \operatorname{Sing}(G) \longrightarrow \mathbb{Q}_{\geq 0}$ will be defined. We also make use of the property (1) in Proposition 2.7 to define, for a given sequence of transformations (2.15), new functions:

$$
\operatorname{Ord}^{(d-e)}\left(G_{i}\right): \operatorname{Sing}\left(G_{i}\right) \longrightarrow \mathbb{Q}_{\geq 0} .
$$

To this end, fix $\beta: V^{(d)} \longrightarrow V^{\left(d^{\prime}\right)}$ transversal to $G$ for $d^{\prime}=d-e$. This induces a sequence (2.16). Set, for each index $i \leq r$,

$$
\operatorname{Ord}^{(d-e)}\left(G_{i}\right)(x)=\operatorname{ord}\left(\left(\mathcal{R}_{G, \beta}\right)_{i}\right)\left(\beta_{i}(x)\right) .
$$

In 2.13, we indicate some general properties of the function $\operatorname{ord}\left(\left(\mathcal{R}_{\mathcal{G}, \beta}\right)\right)$.

Claim 2.10. The function $\mathrm{H}-\operatorname{ord}^{(d-e)}\left(G_{i}\right)(x)$ will be defined so that, for any $x \in \operatorname{Sing}(G)$,

$$
\mathrm{H}-\operatorname{ord}^{(d-e)}\left(G_{i}\right)(x) \leq \operatorname{Ord}^{(d-e)}\left(G_{i}\right)(x) .
$$

That is, the upper semi-continuous function $\operatorname{Ord}^{(d-e)}$ will provide an upper bound for our H-function.

2.13. Note that the functions $\operatorname{Ord}^{(d-e)}\left(G_{i}\right)$ have been studied in [9]. The main results are as follows:

(1) The functions are intrinsic to the sequence of transformations (2.15). Namely, they are independent of the choice of the transversal morphism $\beta: V^{(d)} \rightarrow V^{\left(d^{\prime}\right)}$ in (2.16) ([9, Theorem 10.1]).

(2) The functions $\mathrm{H}$-ord ${ }^{(d-e)}(G)$ and $\operatorname{Ord}^{(d-e)}(G)$ coincide if the characteristic is zero. In particular, the function $\mathrm{H}$-ord ${ }^{(d-e)}(\mathcal{G})$ is upper semicontinuous in such a case. 
(3) It is proved in [9, Part 5 and 10.4] that, up to an induction hypothesis on $d-e$, a sequence (2.16) can be constructed so that either the singular locus of the transform is empty, the $\tau$-invariant increases, or the elimination algebra $\left(\mathcal{R}_{G, \beta}\right)_{r}$ is monomial, that is, it is defined by an invertible sheaf of ideals supported on the exceptional locus, for instance,

$$
\left(\mathcal{R}_{G, \beta}\right)_{r}=\mathcal{O}_{V_{r}^{(d-e)}}\left[I\left(H_{1}\right)^{\alpha_{1}} \ldots I\left(H_{r}\right)^{\alpha_{r}} W^{s}\right],
$$

where $H_{i}$ is the strict transform of the exceptional component introduced by $\pi_{\beta_{i-1}\left(Y_{i-1}\right)}$.

Remark 2.11. In characteristic zero, it is easy to extend a sequence that is monomial (in the sense of (2.18)) to a resolution of singularities. This extension can be constructed by choosing centers in a simple combinatorial manner. However, this is not the case in positive characteristic, which is quite a bit more involved (see Theorem 2.13).

2.14. On the lower-bound function and tamed H-functions. A remarkable property of the $\mathrm{H}$-functions, which was studied in $[6,7.4]$, is that they enable us to assign, to a sequence of transformations of $\mathcal{G}$, a monomial algebra that turns out to be a useful tool in the study of singularities. The monomial algebra assigned to $(2.16)$ is

$$
\mathcal{M}_{r} W^{s}=\mathcal{O}_{V_{r}^{(d-e)}}\left[I\left(H_{1}\right)^{h_{1}} \ldots I\left(H_{r}\right)^{h_{r}} W^{s}\right]
$$

where each exponent $h_{i}$ is defined by setting, for $i=0, \ldots, r-1$,

$$
\frac{h_{i+1}}{S}=\text { H-ord }^{(d-e)}\left(G_{i}\right)\left(\xi_{Y_{i}}\right)-1,
$$

where $\xi_{Y_{i}}$ denotes the generic point of $Y_{i}$.

Claim 2.12. The following inequalities hold for any sequence such as that on (2.15):

$$
\operatorname{ord}\left(\mathcal{M}_{r} W^{s}\right)(x) \leq \mathrm{H}-\operatorname{ord}^{(d-e)}\left(G_{r}\right)(x) \leq \operatorname{Ord}^{(d-e)}\left(G_{r}\right)(x),
$$

and for all $x \in \operatorname{Sing}\left(G_{r}\right)$.

This indicates that at least our untraceable $\mathrm{H}$-function fits between two upper semi-continuous functions. The next theorem gives conditions under which the $\mathrm{H}$-function has a nice and tame behavior. Moreover, when these conditions hold, this leads to a resolution of the algebra.

Theorem 2.13. Fix a differential Rees algebra G. Fix a positive integer e, and assume, as in 2.12, that $\tau_{\mathcal{G}}(x) \geq e$ at any closed point $x \in \operatorname{Sing}(G)$. Consider 
a sequence of transformations (2.16) with the property in (2.18), namely, that the elimination algebra is monomial. Set $\mathcal{M}_{r} W^{s}$ as in (2.19). If, for any $x \in \operatorname{Sing}\left(G_{r}\right)$,

$$
\text { H-ord }{ }^{(d-e)}\left(G_{r}\right)(x)=\operatorname{ord}\left(\mathcal{M}_{r} W^{s}\right)(x),
$$

then the combinatorial resolution of $\mathcal{M}_{r} W^{s}$ can be lifted to a sequence of monoidal transformations over $G_{r}$, for instance,

$$
\begin{array}{ccccc}
\mathcal{G} & G_{1} & G_{r} & G_{N} \\
V^{(d)} \stackrel{\pi_{Y}}{\longleftarrow} V_{1}^{(d)} \stackrel{\pi_{Y_{1}}}{\longleftarrow} \cdots \stackrel{\pi_{Y_{r-1}}}{\longleftarrow} V_{r}^{(d)} \stackrel{\pi_{Y_{r}}}{\longleftarrow} \cdots \stackrel{\pi_{Y_{N-1}}}{\longleftarrow} V_{N}^{(d)},
\end{array}
$$

which is either a resolution of $G$ (i.e., $\operatorname{Sing}(G)=\varnothing)$, or is a case where the $\tau$-invariant improves, that is, $\tau_{G_{N}}(x) \geq e+1$ at any closed point $x \in \operatorname{Sing}\left(G_{N}\right)$ (improvement of the $T$-invariant).

This theorem extends [6, Theorem 8.13], where the case $e=1$ is treated. The theorem and its proof will be addressed here in Theorem 7.3 (see also Remark 7.2).

Definition 2.14. When the setting of Theorem 2.13 holds, namely, when the equality in (2.20) holds at any $x \in \operatorname{Sing}\left(\mathcal{G}_{r}\right)$, then $\mathcal{G}_{r}$ is said to be in the strong monomial case (in other words, H-ord ${ }^{(d-e)}\left(G_{r}\right)$ is tamed).

2.15. On the definition of the functions $\mathrm{H}$-ord ${ }^{(d-e)}$. We have indicated, so far, that these $\mathrm{H}$-functions will be bounded between two upper semi-continuous functions. The results in this paper aim to compute explicitly the function in positive characteristic. This will be achieved through the notion of presentations, which we discuss below.

To clarify our strategy, let us first indicate what is known in the case $e=1$ $(d-e=d-1)$. Given a diagram as (2.16), locally at any point $x \in \operatorname{Sing}\left(G_{r}\right)$, a presentation is defined as

$$
\mathcal{P}=\mathcal{P}\left(\beta_{r}, z, f_{n}(z)\right)
$$

where

- $\{z=0\}$ is a $\beta_{r}$-section (i.e., $\{z=0\}$ is a section of $\left.\beta_{r}: V_{r}^{(d)} \longrightarrow V_{r}^{\left(d^{\prime}\right)}\right)$.

- $f_{n}(z)=z^{n}+a_{1} z^{n-1}+\cdots+a_{n} \in \mathcal{O}_{V^{(d-1)}}[z]$ and $f_{n}(z) W^{n} \in G_{r}$.

Moreover, a presentation can be defined at $x$ so that

$$
\text { H-ord }{ }^{(d-1)}\left(G_{r}\right)(x)=\min _{1 \leq j \leq n}\left\{\frac{v_{\beta_{r}(x)}\left(a_{j}\right)}{j}, \operatorname{ord}\left(\left(\mathcal{R}_{G, \beta}\right)_{r}\right)\left(\beta_{r}(x)\right)\right\} .
$$

In fact, the presentations of the form (2.22) were the tool which led us to the proof of Theorem 5.10 for the case $e=1$ (see [6, Theorem A.7]). We shall indicate in 6.2 how the previous result will lead to the following statement. 
Suppose that $d^{\prime}=d-e$ now for $e>1$. Locally, at any $x \in \operatorname{Sing}\left(G_{r}\right)$, there is a presentation, for instance,

$$
\mathcal{P}=\mathcal{P}\left(\beta_{r}, z_{1}, \ldots, z_{e}, g_{n_{1}}\left(z_{1}\right), \ldots, g_{n_{e}}\left(z_{e}\right)\right),
$$

where

- $\left\{z_{1}=\cdots=z_{e}=0\right\}$ is a $\beta_{r}$-section.

- $g_{n_{i}}\left(z_{i}\right) W^{n_{i}} \in G_{r}$, and for each index, these functions are of the form

$$
\begin{gathered}
g_{n_{1}}\left(z_{1}\right)=z_{1}^{n_{1}}+a_{1}^{(1)} z_{1}^{n_{1}-1}+\cdots+a_{n_{1}}^{(1)} \in \mathcal{O}_{V^{(d-1)}}\left[z_{1}\right], \\
g_{n_{2}}\left(z_{2}\right)=z_{2}^{n_{2}}+a_{1}^{(2)} z_{2}^{n_{2}-2}+\cdots+a_{n_{2}}^{(2)} \in \mathcal{O}_{V^{(d-2)}}\left[z_{2}\right], \\
\vdots \\
\left.g_{n_{e}}\left(z_{r}\right)=z_{r}^{n_{e}}+a_{1}^{(e)} z_{r}^{n_{e}-1}+\cdots+a_{n_{e}}^{(e)} \in \mathcal{O}_{V^{(d-e)}}\right]\left[z_{e}\right] .
\end{gathered}
$$

However, this kind of presentation is not well suited to obtain an expression as that in (2.23), since the coefficients are not all in dimension $d-e$.

In this paper, we overcome this difficulty by proving the existence of the socalled simplified presentations, for instance,

$$
s \mathcal{P}=s \mathcal{P}\left(\beta_{r}, z_{1}, \ldots, z_{e}, f_{n_{1}}\left(z_{1}\right), \ldots, f_{n_{e}}\left(z_{e}\right)\right),
$$

which fulfill the following additional condition:

$$
\begin{aligned}
f_{n_{1}}\left(z_{1}\right) & =z_{1}^{n_{1}}+a_{1}^{(1)} z_{1}^{n_{1}-1}+\cdots+a_{n_{1}}^{(1)} \in \mathcal{O}_{V^{(d-e)}}\left[z_{1}\right], \\
f_{n_{2}}\left(z_{2}\right) & =z_{2}^{n_{2}}+a_{1}^{(2)} z_{2}^{n_{2}-2}+\cdots+a_{n_{2}}^{(2)} \in \mathcal{O}_{V^{(d-e)}}\left[z_{2}\right], \\
\vdots & \\
f_{n_{e}}\left(z_{r}\right) & \left.=z_{r}^{n_{e}}+a_{1}^{(e)} z_{r}^{n_{e}-1}+\cdots+a_{n_{e}}^{(e)} \in \mathcal{O}_{V^{(d-e)}}\right]\left[z_{e}\right] .
\end{aligned}
$$

These simplified presentations are, to some extent, a generalization of the Weierstrass Preparation Theorem. Note that, as opposed to (2.24), all coefficients are now in dimension $d-e$; that is, $a_{j_{i}}^{(i)} \in \mathcal{O}_{V^{(d-e)}}$. This latter property will enable us to extend the previous assertion, namely, that the value of the $\mathrm{H}$-function at $x$ is

$$
\mathrm{H}-\operatorname{ord}^{(d-e)}\left(G_{r}\right)(x)=\min _{\substack{1 \leq j_{i} \leq n_{i} \\ 1 \leq i \leq e}}\left\{\frac{v_{\beta_{r}(x)}\left(a_{j_{i}}^{(i)}\right)}{j_{i}}, \operatorname{ord}\left(\left(\mathcal{R}_{\mathcal{G}, \beta}\right)_{r}\right)\left(\beta_{r}(x)\right)\right\}
$$

Construction of this kind of presentation appears in Theorem 6.5. Simplified presentations will allow us to view each equation in (2.25) as a polynomial in one 
different variable. By using this latter fact, the arguments known for $d^{\prime}=d-1$ (namely (2.23)) will be extended to arbitrary $d^{\prime}$ (e.g., (2.26)).

In particular, these simplified presentations will be used to extend the main results in [6, Main Theorem 1 and Theorem 8.13], and give numerical conditions in terms of the $\mathrm{H}$-functions, under which the sequence (2.16) can be easily extended to a resolution.

The main tool used throughout this paper comprises the differential Rees algebras and their properties. The hope is that a better understanding of these algebras will lead to the conditions in Theorem 2.13, and hence to resolution of singularities. Here, we mean "resolution" in the sense of Hironaka, namely by successive blow-ups along centers included in the closed Hilbert-Samuel stratum (i.e., not in the simplified form introduced in [21]).

In [7], the resolution of surfaces embedded in a three-dimensional ambient space is proved. The techniques developed in this paper will enable us to extend this result to prove the embedded resolution of a two-dimensional scheme embedded in arbitrary dimension (see Theorem 7.4). This last result has also been obtained by other authors in [1], [13] (arithmetic case!), and [18].

\section{WEAK EQUIVALENCE}

3.1. The word invariant was already used in the previous discussion. Hironaka provides this notion with a very precise meaning, which is also related to his notion of equivalence. This notion is discussed below.

In fact, here an invariant of a singular point will be a value assigned to it, and subject to very precise conditions expressed in terms of this equivalence. It will be proved that the value of the $\mathrm{H}$-function at a singular point, discussed in this paper, fulfills these conditions.

Briefly speaking, the equivalence imposes conditions that are related to the form of compatibility with monoidal transformations and smooth morphisms.

3.2. Fix on the smooth scheme $V^{(d)}$ a set of smooth hypersurfaces with normal crossings (e.g., $E=\left\{H_{1}, \ldots, H_{r}\right\}$ ). Let $G=\bigoplus I_{n} W^{n}$ be a Rees algebra over $V^{(d)}$, and let

$$
V^{(d)} \stackrel{\pi}{\longleftarrow} U
$$

be a smooth morphism.

There is a natural notion of pull-backs of the Rees algebra $G$ and the set $E$, for instance, to a Rees algebra $G_{U}$ and a set $E_{U}$. Here, $E_{U}$ is the collection obtained as the pull-backs of the hypersurfaces in $E$. The Rees algebra $G_{U}$ is defined by setting $G_{U}=\bigoplus\left(I_{n}\right)_{U} W^{n}$ to be the natural lifting of $G$ to $U$. This notion of pull-back will be denoted by

$$
\begin{aligned}
& V^{(d)} \longleftarrow \pi \\
& G, E
\end{aligned}
$$


Observe here that the singular locus of the Rees algebra $G$ is compatible with pull-backs; that is,

$$
\operatorname{Sing}\left(G_{U}\right)=\pi^{-1}(\operatorname{Sing}(G)) .
$$

Definition 3.1. Given $G$ and $E$ as above, a local sequence of $G$ and $E$ is a sequence

$$
\begin{aligned}
& V^{(d)} \longleftarrow \pi_{1} \tilde{V}_{1}^{(d)} \longleftarrow \pi_{2} \cdots \stackrel{\pi_{r}}{\longleftarrow} \tilde{V}_{r}^{(d)} \\
& G, E \quad G_{1}, E_{1} \quad G_{r}, E_{r}
\end{aligned}
$$

where each $\tilde{V}_{i}^{(d)} \stackrel{\pi_{i+1}}{\tilde{V}_{i+1}^{(d)}}$ is either a pull-back or a monoidal transformation along a center $C_{i} \subset \operatorname{Sing}\left(G_{i}\right)$ that has normal crossing with the union of the exceptional hypersurfaces in $E_{i}$, for $i=0, \ldots, r-1$. In this latter case, we set $E_{i+1}$ to be the union of the strict transforms of hypersurfaces in $E_{i}$, together with the exceptional locus of the monoidal transformation.

Definition 3.2. Fix two Rees algebras $G$ and $G^{\prime}$ and a set of exceptional hypersurfaces $E$ in the smooth scheme $V^{(d)}$. We say that $G$ and $\mathcal{G}^{\prime}$ are weakly equivalent if

(i) $\operatorname{Sing}(G)=\operatorname{Sing}\left(G^{\prime}\right)$;

(ii) a local sequence of $G$, for instance,

$$
\begin{aligned}
& V^{(d)} \stackrel{\pi_{1}}{\longleftarrow} \tilde{V}_{1}^{(d)} \stackrel{\pi_{2}}{\longleftarrow} \cdots \stackrel{\pi_{r}}{\longleftarrow} \tilde{V}_{r}^{(d)} \\
& G, E \quad G_{1}, E_{1} \quad G_{r}, E_{r} \text {, }
\end{aligned}
$$

defines a local sequence of $\mathcal{G}^{\prime}$ (and vice-versa), and $\operatorname{Sing}\left(G_{i}\right)=\operatorname{Sing}\left(G_{i}^{\prime}\right)$ for $i=0, \ldots, r$.

Remark 3.3. The notion of the weak equivalence is preserved by a local sequence; namely, if $G$ and $G^{\prime}$ are weakly equivalent as before, then also their transforms $G_{r}$ and $G_{r}^{\prime}$ are weakly equivalent.

A first example of equivalence arises when we consider the integral closure of a Rees algebra $\mathcal{G}$. In fact, if $\tilde{\mathcal{G}}$ denotes the integral closure of $\mathcal{G}$, then $\tilde{\mathcal{G}}$ is again a Rees algebra. The following theorem includes a relation of weak equivalence between $\mathcal{G}$ and $\tilde{G}$.

\section{Theorem 3.4.}

(1) Fix two Rees algebras $\mathcal{G}$ and $\tilde{G}$ with the same integral closure. Then, $G$ and $\tilde{G}$ are weakly equivalent.

(2) Fix a Rees algebra $G$. Let $\operatorname{Diff}(G)$ be the differential Rees algebra attached to $\mathcal{G}$ (see 2.8). Then, $\mathcal{G}$ and $\operatorname{Diff}(\mathcal{G})$ are weakly equivalent.

Proof.

(1) See [22, Proposition 5.4].

(2) This property is also known as Giraud's lemma (for more, see [22, Theorem 4.1]). 
3.3. We address here the notion of invariant, as needed in our development. Given $G$ over $V^{(d)}$, an invariant attached to $x \in \operatorname{Sing}(\mathcal{G})$ is a value (e.g., $\gamma(x, \mathcal{G})$ ) that is subject to the condition

$$
\gamma(x, \mathcal{G})=\gamma\left(x, G^{\prime}\right)
$$

whenever $\mathcal{G}$ and $\mathcal{G}^{\prime}$ are weakly equivalent when restricted to some neighborhood of $x$.

A first example of invariant is that of the value $\boldsymbol{\tau}_{\mathcal{G}}(x)$ in 2.7 (see also [44] and [4]). It will be proved here in Theorem 6.9(1) that the value $\mathrm{H}$-ord ${ }^{(r)}(G)(x)$ is an invariant, for any point $x \in \operatorname{Sing}(G)$.

\section{The Slope of a Hypersurface and the WeaK Equivalence}

In this section, we restrict attention to the description of the $(d-1)$-dimensional $\mathrm{H}$-function at a point in the highest multiplicity locus of a hypersurface. In such a context, this value, introduced by Hironaka, is a rational number that is defined as an optimal slope. We present here the slope as a refinement of the multiplicity at the point (see Definition 4.1). First, this will be done in terms of a transversal projection; then, it will be shown to be independent of the choice of such a projection (Theorem 4.10).

4.1. Slope of a monic polynomial. Fix a hypersurface $X$ embedded in a smooth scheme $V^{(d)}$ and a closed $n$-fold point $x$ of $X$ (i.e., a point of multiplicity $n)$. After suitable restriction, in étale topology, a smooth morphism $V^{(d)} \stackrel{\beta}{\longrightarrow}$ $V^{(d-1)}$ can be defined so that $X$ is expressed by a monic polynomial of degree $n$, of the form

$$
f_{n}(z)=z^{n}+a_{1} z^{n-1}+\cdots+a_{n} \in \mathcal{O}_{V^{(d-1)}}[z],
$$

where $z$ is a global section of $\mathcal{O}_{V^{(d)}}$, and $z=0$ is a $\beta$-section. We abuse notation and say that $z$ is a transversal section of $\beta$. This says that, after suitable restriction in étale topology, both at $x$ and $\beta(x)$, the restricted map $\left.\beta\right|_{X}$

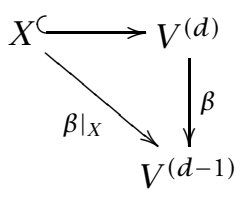

is finite. Thus, locally at any $y \in V^{(d-1)}$, we may view $X$ as defined by the monic polynomial in (4.1). In particular, the fiber over $y$ (e.g., $\left.\beta^{-1}(y)\right)$ is given by $\bar{f}_{n}(z)=z^{n}+\bar{a}_{1} z^{n-1}+\cdots+\bar{a}_{n} \in k(y)[z]$, where $k(y)$ is the residue field at the point. 
To simplify notation, let $f_{n} W^{n}$ denote the Rees algebra $\mathcal{O}_{V^{(d)}}\left[f_{n}(z) W^{n}\right]$. Note that $\operatorname{Sing}\left(f_{n} W^{n}\right)$ is the set of $n$-fold points of the hypersurface. Here, we will assume that $\operatorname{Sing}\left(f_{n} W^{n}\right)$ has no components of codimension one in $V^{(d)}$, and this will ensure that $f_{n}(z) \neq z^{n}$ for any expression as (4.1).

Definition 4.1. Assume, as before, that $X$ is defined by the monic polynomial in (4.1). The slope of $f_{n}(z) W^{n}$ at $y \in V^{(d-1)}$ is the rational number

$$
\operatorname{Sl}\left(f_{n}(z) W^{n}\right)(y)=\min _{1 \leq j \leq n}\left\{\frac{v_{y}\left(a_{j}\right)}{j}\right\} .
$$

Geometrically, the slope defined by the equation (4.1) is the biggest rational number $q$ so that all pairs $\left(v_{y}\left(a_{j}\right), n-j\right)$ lie above the line through $(0, n)$ and $(n q, 0)$ :

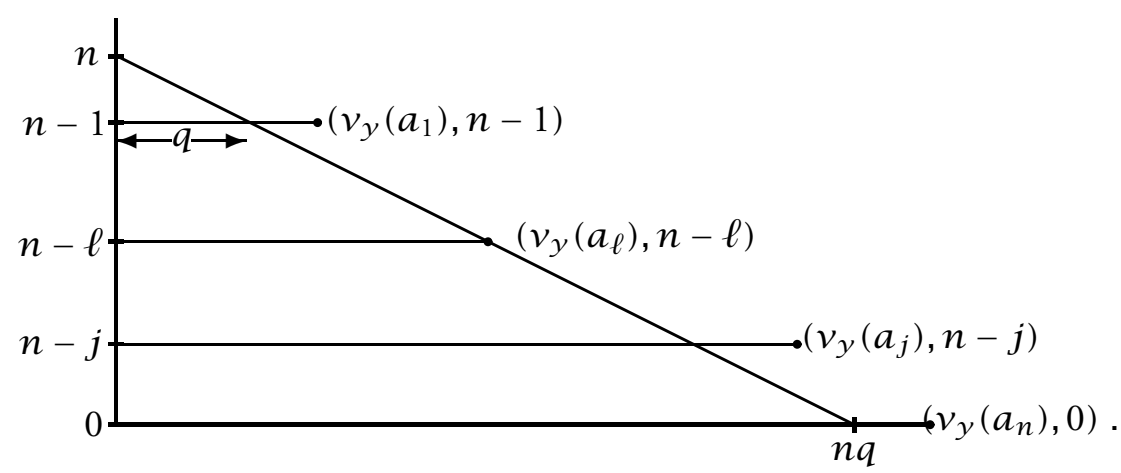

Changes on the variable $z$ imply changes on (4.1), and hence on the value (4.3). We aim to find the biggest possible value of the slope at the fixed point $y$. A first step in this direction will be addressed in Remark 4.6.

The following technical lemma and remark gather some results to be used in this and further sections, which will be needed to establish the optimality of the rational number.

Lemma 4.2 (Zariski's Multiplicity Lemma [52, Chapter VIII Section 10, Corollary 1 to Theorem 24]). Let $f_{n}$ be as in (4.1), with $X$ defined as in (4.2). Then, the following hold:

(1) Fix $y \in \beta\left(\operatorname{Sing}\left(f_{n} W^{n}\right)\right)$. Then, $\bar{f}_{n}(z)=(z-\bar{\alpha})^{n} \in k(y)[z]$, for a suitable $\bar{\alpha} \in k(y)$, the residue field at $\mathcal{O}_{V^{(d-1)}, y}$. In particular, the fiber $\beta^{-1}(y)$ has a unique point which is rational.

(2) Assume that $C \subset \operatorname{Sing}\left(f_{n} W^{n}\right)$ is smooth and irreducible. $\beta(C)$ is smooth and, moreover, $\left.\beta\right|_{C}$ induces an isomorphism $\left.\beta\right|_{C}: C \stackrel{\cong}{\rightrightarrows} \beta(C)$ (see Proposition 2.7 (3)). In particular, if $V^{(d-1)}$ is affine, there is a global section $\alpha \in \mathcal{O}_{V^{(d-1)}}$ so that $(z-\alpha) \in I(C) \subset \mathcal{O}_{V^{(d)}}$. 
(3) Let $C^{\prime}$ be a smooth and irreducible component of $\beta\left(\operatorname{Sing}\left(f_{n} W^{n}\right)\right)$. There is a unique irreducible component $C \subset \operatorname{Sing}\left(f_{n} W^{n}\right)$ such that $\beta(C)=C^{\prime}$. Moreover, $C$ and $C^{\prime}$ are isomorphic, and hence $C$ is smooth.

Further details can be found in [48], [49], and [9]. In fact, parts (1), (2), and (3) are a byproduct of the Zariski Multiplicity Lemma. Note that (1) says that $\left.\beta\right|_{\operatorname{Sing}\left(f_{n} W^{n}\right)}: \operatorname{Sing}\left(f_{n} W^{n}\right) \longrightarrow \beta\left(\operatorname{Sing}\left(f_{n} W^{n}\right)\right)$ is a set theoretical bijection, and that corresponding points have the same residue field. Part (2) and the first half of part (3) follow essentially from this fact. As for the second half of part (3), note that $\left.\beta\right|_{C}: C \longrightarrow \beta(C)=C^{\prime}$ is a finite birational morphism, and that $C^{\prime}$ is normal.

\section{Remark 4.3.}

(1) Fix $y \in V^{(d-1)}$. Let $\bar{f}_{n}(z)=z^{n}+\bar{a}_{1} z^{n-1}+\cdots+\bar{a}_{n} \in k(y)[z]$ be the class of $f_{n}(z)$ on the fiber. Then, $\operatorname{Sl}\left(f_{n}(z) W^{n}\right)(y)=0$ if and only if $\bar{f}_{n}(z) \neq z^{n}$.

When $y \in \beta\left(\operatorname{Sing}\left(f_{n} W^{n}\right)\right)$, then $\bar{f}_{n}(z)=(z-\bar{\alpha})^{n}$. A suitable change of variables of the form $z_{1}=z+\gamma$, with $\gamma \in \mathcal{O}_{V^{(d-1)}, y}$, can be defined with the property that $\bar{\gamma}=\bar{\alpha}$ in $k(y)$. In particular, the restriction to the fiber over $y$ is $\bar{f}_{n}^{\prime}\left(z_{1}\right)=z_{1}^{n}$, and hence $\operatorname{Sl}\left(f_{n}^{\prime}\left(z_{1}\right)\right)(y)>0$. Moreover, we claim that in this case $\operatorname{Sl}\left(f_{n}^{\prime}\left(z_{1}\right)\right)(y) \geq 1$. To check this, notice that the condition $\operatorname{Sl}\left(f_{n}^{\prime}\left(z_{1}\right)\right)(y)>0$ implies there is a unique point of $X=V\left(f_{n}^{\prime}\left(z_{1}\right)\right)$ (e.g., $\left.y^{\prime} \in V^{(d)}\right)$ dominating $y$. If $\left\{x_{1}, \ldots, x_{\ell}\right\}$ is a regular system of parameters at $\mathcal{O}_{V^{(d-1)}, y}$, then $\left\{z_{1}, x_{1}, \ldots, x_{\ell}\right\}$ is a regular system of parameters at $\mathcal{O}_{V^{(d)}, y^{\prime}}$. As $f_{n}^{\prime}\left(z_{1}\right)$ has multiplicity $n$ at $\mathcal{O}_{V^{(d)}, y^{\prime}}$, it follows that $f_{n}^{\prime}\left(z_{1}\right)=z_{1}^{n}+b_{1} z^{n-1}+\cdots+b_{n} \in\left\langle z_{1}, x_{1}, \ldots, x_{\ell}\right\rangle^{n}$, and hence each $b_{i} \in\left\langle x_{1}, \ldots, x_{\ell}\right\rangle^{i}$.

(2) We fix $x \in C \subset \operatorname{Sing}\left(f_{n} W^{n}\right)$ and set $\mathbf{x}=\beta(x)$. One can argue as in Lemma 4.2(2) to show that after a suitable change of the form $z_{1}=z-\alpha$, with $\alpha \in \mathcal{O}_{V^{(d-1)}, \mathbf{x}}$, one obtains $\operatorname{Sl}\left(f_{n}^{\prime}\left(z_{1}\right) W^{n}\right)(y)>0$. Here, $y$ denotes the generic point of $\beta(C)$. In particular, $\operatorname{Sl}\left(f_{n}^{\prime}\left(z_{1}\right) W^{n}\right)(y) \geq 1$.

(3) The previous discussion applies also when we fix $x \in C^{\prime} \subset \beta\left(\operatorname{Sing}\left(f_{n} W^{n}\right)\right)$, with $C^{\prime}$ smooth and irreducible. In fact, a suitable change of variables of the form $z_{1}=z+\alpha$ with $\alpha \in \mathcal{O}_{C^{\prime}, x}$ (locally at $x$ ) can be considered so that $\operatorname{Sl}\left(f_{n}^{\prime}\left(z_{1}\right) W^{n}\right)(y)>0$, where $y$ denotes the generic point of $C^{\prime}$. Moreover, part (1) implies that $\operatorname{Sl}\left(f_{n}^{\prime}\left(z_{1}\right) W^{n}\right)(y) \geq 1$.

We will now provide a criterium that will ensure conditions under which the rational number $\operatorname{Sl}\left(f_{n}(z) W^{n}\right)(y)$ is optimal.

Definition 4.4. Fix a hypersurface embedded in a $d$-dimensional scheme, $X \subset V^{(d)}$, a smooth morphism $V^{(d)} \stackrel{\beta}{\longrightarrow} V^{(d-1)}$, and a transversal parameter $z$ so that $f_{n}(z)=z^{n}+a_{1} z^{n-1}+\cdots+a_{n} \in \mathcal{O}_{V^{(d-1)}}[z]$ defines the hypersurface $X$ after a suitable restriction. Fix $y \in V^{(d-1)}$, and set $q=\operatorname{Sl}\left(f_{n}(z) W^{n}\right)(y) \in \mathbb{Q}$. Let $r_{j}=v_{y}\left(a_{j}\right)(j=1, \ldots, n)$ denote the order of each coefficient at $\mathcal{O}_{V^{(d-1)}, y}$. Fix a regular system of coordinates at $\mathcal{O}_{V^{(d-1)}, y}$ (e.g., $\left.\left\{x_{1}, \ldots, x_{\ell-1}\right\}\right)$. At the completion (e.g., $\left.\hat{\mathcal{O}}_{V^{(d-1)}, y}=k(y) \llbracket x_{1}, \ldots, x_{\ell-1} \rrbracket\right)$, define $f_{n}(z)=z^{n}+\hat{a}_{1} z^{n-1}+\cdots+\hat{a}_{n}$, 
with

$$
\hat{a}_{j}=\sum_{i \geq r_{j}} A_{j}^{i} \in k(y) \llbracket x_{1}, \ldots, x_{\ell-1} \rrbracket,
$$

where $A_{j}^{i}$ is a homogeneous polynomial of degree $i$ in the variables $x_{1}, \ldots, x_{\ell-1}$. Here, $\ell=d-1$ if $y$ is a closed point in $V^{(d)}$.

The weighted initial form of $f_{n}(z)$ (over $y$ ) is defined as

$$
\mathbf{w}-\mathbf{i n} \mathbf{n}_{y}\left(f_{n}(z)\right)=\sum_{0 \leq j \leq n} A_{j}^{j q} Z^{n-j} \in \operatorname{gr}\left(\mathcal{O}_{V^{(d-1)}}, y\right)[Z],
$$

where each $A_{j}^{j q}$ is weighted homogeneous of degree $j q$, and where $A_{j}^{j q}=0$ if $j q \notin \mathbb{Z}$.

Note that $\mathbf{w}-\mathbf{i n} \mathbf{n}_{y}\left(f_{n}(z)\right)$ is defined in $\operatorname{gr}\left(\mathcal{O}_{V^{(d-1)}, y}\right)[Z]$, and it is weighted homogeneous of degree $n q$ if $Z$ is given weight $q$ and each $X_{i}=\operatorname{In}\left(x_{i}\right)$ has weight 1 for $i=1, \ldots, d-1$. To ease notation, we provide $z$ with weight $q$ and each $x_{i}$ with weight 1 at the ring of formal power series.

Remark 4.5. Suppose that $\operatorname{Sl}\left(f_{n}(z) W^{n}\right)(y)=0$. Then, w-in $y_{y}\left(f_{n}(z)\right)$ is weighted homogeneous of degree 0 if $z$ is endowed with weight $q=0$ and each $x_{i}$ is given weight 1 . In such a case, $\mathbf{w}$-in $\mathbf{n}_{y}\left(f_{n}(z)\right)$ is defined in $k(y)[Z]$, and there is a natural identification of this polynomial with the equation that defines the fiber of the hypersurface over the point $y$, namely, w-in $y\left(f_{n}(z)\right)=\bar{f}_{n}(Z) \in$ $k(y)[Z]$, where $\bar{f}_{n}(z)$ is the equation that defines the fiber.

Remark 4.6. Note that in (4.4) we have $A_{0}^{0}=1$, and hence $\mathbf{w}$-in ${ }_{y}\left(f_{n}(z)\right)$ is a monic polynomial of degree $n$. Moreover, we claim that $\mathbf{w}-\mathbf{i n}_{y}\left(f_{n}(z)\right) \neq Z^{n}$.

In fact, one can check that $\mathbf{w}-\mathbf{i n}_{y}\left(f_{n}(z)\right)$ is an $n$-th power if and only if $\mathbf{w}-\mathbf{i n}_{y}\left(f_{n}(z)\right)=(Z+A)^{n}$ for some $A \in \operatorname{gr}\left(\mathcal{O}_{V^{(d-1)}, y}\right)$. In this case, $A$ must be homogeneous of degree $q$. If this occurs, then $q \in \mathbb{Z}$, and hence there is an element $\alpha \in \mathcal{O}_{V^{(d-1)}, y}$ so that $\operatorname{In}_{y}(\alpha)=A$. The change of variables $z_{1}=z+\alpha$ gives rise to a strictly higher slope, that is, $\operatorname{Sl}\left(f_{n}^{\prime}\left(z_{1}\right) W^{n}\right)(y)>\operatorname{Sl}\left(f_{n}(z) W^{n}\right)(y)$.

The previous discussion shows that a change of the form $z_{1}=z+\alpha$ can increase the slope if and only if $\mathbf{w}$-in $\mathbf{i n}_{y}\left(f_{n}(z)\right)$ is an $n$-th power.

As $\operatorname{Sing}\left(f_{n} W^{n}\right)$ has no components of codimension 1 in $V^{(d)}$, by assumption, one can check that after finitely many changes of the variable $z$ as above, we may assume that $\mathbf{w}$-in $\mathbf{n}_{y}\left(f_{n}(z)\right)$ is not an $n$-th power. This leads to the following definition.

Definition 4.7. A monic polynomial $f_{n}(z)$ is said to be in normal form at a point $y \in V^{(d-1)}$ if the weighted initial form $\mathbf{w}-\mathbf{i n}_{y}\left(f_{n}(z) W^{n}\right)$ is not an $n$-th power at $\operatorname{gr}\left(\mathcal{O}_{V^{(d-1)}, y}\right)[Z]$.

Proposition 4.8. Fix a point $y \in V^{(d-1)}$ and a polynomial

$$
f_{n}(z)=z^{n}+a_{1} z^{n-1}+\cdots+a_{n} \in \mathcal{O}_{V^{(d-1)}}[z]
$$


that is in normal form at $y$; that is, assume that $z$ is such that $\mathbf{w}$-in $\mathbf{n}_{y}\left(f_{n}(z)\right)$ is not an $n$-th power at $\operatorname{gr}\left(\mathcal{O}_{V^{(d-1)}, y}\right)[Z]$. Then, $y \in \beta\left(\operatorname{Sing}\left(f_{n} W^{n}\right)\right)$ if and only if $q=\operatorname{SI}\left(f_{n}(z) W^{n}\right)(y) \geq 1$.

Proof. If $y \in \beta\left(\operatorname{Sing}\left(f_{n} W^{n}\right)\right)$, and since $\mathbf{w}$-in $y\left(f_{n}(z)\right)$ is not an $n$-th power, then $\operatorname{Sl}\left(f_{n}(z) W^{n}\right)(y)>0$. Under these conditions, Remark 4.3(1) says that $\operatorname{Sl}\left(f_{n}(z) W^{n}\right)(y) \geq 1$.

Conversely, suppose that $\operatorname{Sl}\left(f_{n}(z) W^{n}\right)(y) \geq 1$. Lemma 4.2(1) says that $\operatorname{Sl}\left(f_{n}(z) W^{n}\right)(y)>0$ implies there is a unique point (e.g., $y^{\prime} \in X=V\left(f_{n}(z)\right)$ ) dominating $y$. Fix a regular system of parameters $\left\{x_{1}, \ldots, x_{\ell}\right\}$ at $\mathcal{O}_{V^{(d-1)}, y}$, and recall that $\left\{z, x_{1}, \ldots, x_{\ell}\right\}$ is a regular system of parameters at $\mathcal{O}_{V^{(d)}, y^{\prime}}$. Finally, the condition $\operatorname{Sl}\left(f_{n}(z) W^{n}\right)(y) \geq 1$ implies that $v_{y}\left(a_{i}\right) \geq i$, and hence that $y^{\prime}$ is an $n$-fold point of $X$.

Remark 4.9. We let the assumptions be as in (4.2), where we have that $f_{n}(z)=z^{n}+a_{1} z^{n-1}+\cdots+a_{n} \in \mathcal{O}_{\left.V^{(d-1}, y\right)}[z]$. A function, for instance, $q_{\beta}: \operatorname{Sing}\left(f_{n} W^{n}\right) \longrightarrow \mathbb{Q}>0$, will be defined by setting

$$
q_{\beta}(x):=\max _{z_{1}}\left\{\operatorname{Sl}\left(f_{n}\left(z_{1}\right) W^{n}\right)(\beta(x))\right\},
$$

where $z_{1}=z+\alpha$ for all possible choices of $\alpha \in \mathcal{O}_{V^{(d-1)}, \beta(x)}$ (see Remark 4.3(1)). Note that, if $f_{n}(z)$ is in normal form at $\beta(x)$, then Remark 4.6 ensures that

$$
q_{\beta}(x)=\operatorname{Sl}\left(f_{n}(z) W^{n}\right)(\beta(x)) .
$$

Theorem 4.10. Fix a projection $V^{(d) \stackrel{\beta}{\rightarrow}} V^{(d-1)}$ together with a monic polynomial $f_{n}(z)=z^{n}+a_{1} z^{n-1}+\cdots+a_{n} \in \mathcal{O}_{V^{(d-1)}}[z]$, where $\{z=0\}$ is a section of $\beta$. Consider a point $x \in \operatorname{Sing}\left(f_{n} W^{n}\right)$, and assume that $f_{n}(z)$ is in normal form at $\beta(x)$. Let $q$ denote the slope of $f_{n}(z)$ at $\beta(x)$. The rational number $q$ is completely characterized by the weak equivalence class of the algebra $G=\mathcal{O}_{V^{(d)}}\left[f_{n} W^{n}\right]$ in a neighborhood of $x$.

Corollary 4.11. Fix a hypersurface $X \subset V^{(d)}$ of maximum multiplicity $n$, and two projections $\beta: V^{(d)} \longrightarrow V^{(d-1)}$ and $\beta^{\prime}: V^{(d)} \longrightarrow V^{(d-1)}$, each in the setting of (4.2). For any $n$-fold point $x \in X$,

$$
q_{\beta}(x)=q_{\beta^{\prime}}(x) .
$$

Proof. The rational number $q_{\beta}(x)$ is completely determined in terms of the weak equivalence class of $G=\mathcal{O}_{V^{(d)}}\left[f_{n} W^{n}\right]$ at $x$, and therefore it is independent of the chosen projection.

Proof of Theorem 4.10. The proof of this theorem is based on the so-called Hironaka trick (see $[20,7.1])$.

Set $\mathbf{x}=\beta(x)$. As $x \in \operatorname{Sing}\left(f_{n} W^{n}\right)$, Lemma 4.2(1) then says that we have $\bar{f}_{n}(z)=(z-\bar{\alpha})^{n}$ (with $\left.\alpha \in k(\mathbf{x})\right)$. Here, $\bar{f}_{n}(z)$ denotes the restriction to the 
fiber over $\mathbf{x}$. Since $f_{n}(z)$ is a normal form at $\mathbf{x}$, Remark 4.3(1) ensures that $\bar{\alpha}=0$, and hence $\mathcal{z}$ vanishes at $x$. A regular system of parameters $\left\{x_{1}, \ldots, x_{\ell}\right\}$ at $\mathcal{O}_{V^{(d-1)}, \mathbf{x}}$ can be extended to a regular system of parameters $\left\{z, x_{1}, \ldots, x_{\ell}\right\}$ at $\mathcal{O}_{V^{(d)}, x}$. Note also that $x \in \operatorname{Sing}\left(f_{n} W^{n}\right)$, so $v_{\mathbf{X}}\left(a_{j}\right) \geq j$ for $j=1, \ldots, n$.

Express the monic polynomial as $f_{n}(z)=z^{n}+\hat{a}_{1} z^{n-1}+\cdots+\hat{a}_{n}$ at the completion $\hat{\mathcal{O}}_{V^{(d)}, x}$, and set

$$
\hat{a}_{j}=A_{j}^{j q}+\tilde{A}_{j} \in k(\mathbf{x}) \llbracket x_{1}, \ldots, x_{\ell} \rrbracket,
$$

where $A_{j}^{j q}$ is homogeneous of degree $j q$ and $\tilde{A}_{j}$ has order $>j q$. Here, $A_{j}^{j q}=0$ if $j q \notin \mathbb{Z}$. Recall that $f_{n}(z)$ is in normal form, that is, that

$$
\mathbf{w}-\mathbf{i n}_{\mathbf{x}}\left(f_{n}(z)\right)=Z^{n}+\sum_{j=1}^{n} A_{j}^{j q} Z^{n-j} \in g r_{\mathbf{x}}\left(\mathcal{O}_{V^{(d-1)}}\right)[Z]
$$

is not an $n$-th power. Denote by $r_{j}=v_{\mathbf{X}}\left(a_{j}\right)(j=1, \ldots, n)$ the order of each coefficient at $\mathcal{O}_{V^{(d-1)}, \mathbf{x}}$. Note that $A_{j}^{j q}=0$ if $r_{j} / j>q$.

Stage A: Let $V^{(d)} \times \mathbb{A}^{1}$ denote the product of $V^{(d)}$ with the affine line. The projection on the first coordinate enables us to take the pull-back of $f_{n}(z)$, in a neighborhood of $(x, 0) \in V^{(d)} \times \mathbb{A}^{1}$. The natural extension

$$
V^{(d)} \times \mathbb{A}^{1} \stackrel{\beta \times i d}{\longrightarrow} V^{(d-1)} \times \mathbb{A}^{1}
$$

is a smooth morphism that maps $(x, 0)$ to $(\mathbf{x}, 0)$. The natural identification of

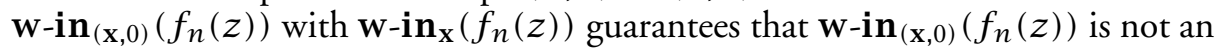
$n$-th power. This identification together with Remark 4.3(1) ensures that

$$
\operatorname{Sl}\left(f_{n}(z) W^{n}\right)((\mathbf{x}, 0))=\operatorname{Sl}\left(f_{n}(z) W^{n}\right)(\mathbf{x})=q \geq 1 .
$$

Fix coordinates $\left\{z, x_{1}, \ldots, x_{e}, t\right\}$ locally at $(x, 0)$; here, $\left\{z, x_{1}, \ldots, x_{e}\right\}$ is the regular system of parameters at $\mathcal{O}_{V^{(d)}, x}$ mentioned before. Consider the monoidal transformation with center $p_{0}=(x, 0)$, and let $p_{1}$ be the intersection of the new exceptional hypersurface (e.g., $H_{1}$ ), with the strict transform of $x \times \mathbb{A}^{1}$.

The point $p_{1}$ can be identified with the origin of the $U_{t}$-chart, where we have $U_{t}=\operatorname{Spec}\left(k\left[z / t, \ldots, x_{e} / t, t\right]\right)$. Now set

$$
f_{n}^{(1)}\left(z_{1}\right)=z_{1}^{n}+t^{r_{1}-1} \tilde{a}_{1}^{(1)} z_{1}^{n-1}+\cdots+t^{r_{n}-n} \tilde{a}_{n}^{(1)}
$$

at the completion of the local ring of at $p_{1}$, and check that

$$
t^{r_{j}-j} \tilde{a}_{j}^{(1)} z_{1}^{n-j}=t^{r_{j}-j}\left(A_{j}^{j q}+t^{\gamma_{j}} \tilde{A}_{j}\right) z_{1}^{n-j}
$$

with $\gamma_{j}>0$ for $j=1, \ldots, n$. Here, $z_{1}=z / t$ and $\tilde{a}_{j}^{(1)}$ is the strict transform of $\hat{a}_{j}$. 
This process can be iterated $N$-times, defining a sequence of monoidal transformations at $p_{1}, \ldots, p_{N-1}$, where each $p_{j}$ is the point of intersection of the new exceptional component (e.g., $H_{j}$ ) with the strict transform of $x \times \mathbb{A}^{1}$.

The final strict transform of $f_{n}(z)$ at the $U_{t}$-chart is given by

$$
f_{n}^{(N)}\left(z_{N}\right)=z_{N}^{n}+t^{N\left(r_{1}-1\right)} \tilde{a}_{1}^{(N)} z_{N}^{n-1}+\cdots+t^{N\left(r_{n}-n\right)} \tilde{a}_{n}^{(N)},
$$

where

$$
t^{N\left(r_{j}-j\right)} \tilde{a}_{j}^{(N)} z_{N}^{n-j}=t^{N\left(r_{j}-j\right)}\left(A_{j}^{j q}+t^{\gamma_{j}^{\prime}} \tilde{A}_{j}\right) z_{N}^{n-j}
$$

with $\gamma_{j}^{\prime}>0$ for $j=1, \ldots, n$.

It may occur after this process of monoidal transformations that the set $\left\{f_{n}^{(N)}=0\right\} \cap H_{N}$ is a two-codimensional component of the $n$-fold points of $\left\{f_{n}^{(N)}=0\right\}$. In that case, we fix $N$ sufficiently large, and look for the largest number of successive monoidal transformations that can be defined with center in codimension 2. We explain below how these centers will be chosen for this new sequence of transformations.

The proof will also show that the rational number $q$ can be characterized in terms of these monoidal transformations.

Stage B: First, consider the monoidal transformation along the center $\left\langle z_{N}, t\right\rangle$, if such a center is permissible. Denote $z_{N} / t$ by $z_{N+1}$ at the $U_{t}$-chart. The transform is

$$
f_{n}^{(N+1)}\left(z_{N+1}\right)=z_{N+1}^{n}+t^{N\left(r_{1}-1\right)-1} \tilde{a}_{1}^{(N+1)} z_{N+1}^{n-1}+\cdots+t^{N\left(r_{n}-n\right)} \tilde{a}_{n}^{(N+1)},
$$

where

$$
t^{N\left(r_{j}-j\right)-j} \tilde{a}_{j}^{(N+1)} z_{N+1}^{n-j}=t^{N\left(r_{j}-j\right)-j}\left(A_{j}^{j q}+t^{\gamma_{j}^{\prime \prime}} \tilde{A}_{j}\right) z_{N+1}^{n-j}
$$

with $\gamma_{j}^{\prime \prime}>0$ for $j=1, \ldots, n$.

After applying $\ell$ monoidal transformations along centers of codimension 2 of the form $\left\langle z_{N+i}, t\right\rangle$, the exponent of $t$ in each coefficient is $N\left(r_{j}-j\right)-\ell j$. Therefore, $\left\langle z_{N+\ell}, t\right\rangle$ is a permissible center whenever $N\left(r_{j}-j\right)-\ell j \geq j$ for all $j \in\{1, \ldots, n\}$. In particular, this condition requires that

$$
\ell \leq \min _{1 \leq j \leq n}\left\{N\left(\frac{r_{j}}{j}-1\right)-1\right\}=N(q-1)-1 .
$$

The geometric interpretation of the previous sequence of $\ell$-monoidal transformations can be described as follows: set $X_{N}=\left\{f_{n}^{(N)}=0\right\}$ (see (4.7)), and let $H_{N}$ denote the exceptional hypersurface $t=0$. The sequence we have previously constructed can be expressed in terms of the diagram

$$
\begin{aligned}
& \begin{array}{lll}
X_{N} & X_{N+1} & X_{N+\ell}
\end{array} \\
& V_{N}^{(d+1)} \stackrel{\pi_{N+1}}{\longleftarrow} V_{N+1}^{(d+1)} \stackrel{\pi_{N+2}}{\ldots} \ldots \stackrel{\pi_{N+\ell}}{\longleftarrow} V_{N+\ell}^{(d+1)} \text {, }
\end{aligned}
$$


where, if $H_{N+i+1}$ denotes the exceptional hypersurface of $\pi_{N+i}$, then the centers of these monoidal transformations are defined by $X_{N+i+1} \cap H_{N+i+1}$.

Let $\tilde{\beta}$ denote the morphism $V^{(d)} \times \mathbb{A}^{1} \longrightarrow V^{(d-1)} \times \mathbb{A}^{1}$ in (4.6). This morphism has a natural lifting to the sequence of monoidal transformations of length $N$ in Stage A, and also to the sequence (4.8). This is guaranteed by Proposition 2.7(3). In particular, for each index $i=0, \ldots, \ell$, the previous sequence defines morphisms

$$
\tilde{\beta}_{N+i}: V_{N+i}^{(d+1)} \longrightarrow V_{N+i}^{(d)} \text {. }
$$

Set

$$
\ell_{N}=\lfloor N(q-1)-1\rfloor .
$$

We finally claim that, for $\ell=\ell_{N}$, we have that $X_{N+\ell} \cap H_{N+\ell}$ is not a permissible center for $X_{N+\ell}$ (see (4.8)).

In fact, whenever $N q \notin \mathbb{Z}$, and after applying $\ell_{N}$ monoidal transformations along these centers of codimension 2, one gets that $V\left(\left\langle z_{N+\ell_{N}}, t\right\rangle\right) \subset X_{N+\ell_{N}}$ and $0<\operatorname{Sl}\left(f_{n}^{\left(N+\ell_{N}\right)} W^{n}\right)\left(\xi_{H}\right)<1$. Thus, $H_{N+\ell_{N}}=\{t=0\}$ cannot be a component of $\tilde{\beta}_{N+\ell}\left(\operatorname{Sing}\left(f_{n}^{\left(N+\ell_{N}\right)} W^{n}\right)\right.$ ) (see Proposition 4.8).

On the other hand, if $N q \in \mathbb{Z}$, then $N(q-1)-1$ is a positive integer. In this case, after applying $N(q-1)-1$ monoidal transformations along these twocodimensional centers, the final strict transform of $f_{n}^{(N)}(z)$ is given by

$$
f_{n}^{\left(N+\ell_{N}\right)}(z)=z_{N+\ell_{N}}^{n}+t^{N\left(r_{1}-q\right)} \tilde{a}_{1}^{\left(N+\ell_{N}\right)} z_{N+\ell_{N}}^{n-1}+\cdots+t^{N\left(r_{n}-q n\right)} \tilde{a}_{n}^{\left(N+\ell_{N}\right)}
$$

at the chart of interest. We claim that $X_{N+\ell_{N}} \cap H_{N+\ell_{N}}$ is not a permissible center.

We note that $\operatorname{Sl}\left(f_{n}^{\left(N+\ell_{N}\right)}\left(z_{N+\ell_{N}}\right) W^{n}\right)\left(\xi_{H}\right)=0$. Remark 4.5 now shows that $\mathbf{w}-\mathbf{i n}_{\xi_{H}}\left(f_{n}^{\left(N+\ell_{N}\right)}\left(z_{N+\ell_{N}}\right)\right)$ can be identified with the equation defining the fiber over $\xi_{H}$ :

$$
\left.f_{n}^{\left(N+\ell_{N}\right)}\left(z_{N+\ell_{N}}\right)\right|_{t=0}=z_{N+\ell_{N}}^{n}+\sum_{j=1}^{n} A_{j}^{j q} z_{N+\ell_{N}}^{n-j} .
$$

The expression of the right-hand side can be identified with $\mathbf{w}-\mathbf{i n}_{\mathbf{x}}\left(f_{n}(z)\right)$ in a natural way (see (4.5)). As we assume that $\mathbf{w}$-in $\mathbf{i n}_{\mathbf{x}}\left(f_{n}(z)\right)$ is not an $n$-th power, Proposition 4.8 together with Remark 4.3(3) ensure that $H_{N+\ell_{N}}$ is not a component of $\tilde{\beta}_{N+\ell_{N}}\left(\operatorname{Sing}\left(f_{n}^{\left(N+\ell_{N}\right)} W^{n}\right)\right)$.

In Remark 4.12 we will apply the following discussion to show that $q$ is totally characterized by Hironaka's weak equivalence class of the $n$-fold points of the hypersurface $\left\{f_{n}=0\right\}$. Notice that the construction of the sequences in Stage A and Stage B, together with (4.10), leads to the equality

$$
\lim _{N \rightarrow \infty} \frac{\ell_{N}}{N}=q-1
$$


Remark 4.12. We claim that the rational number $q$ can be expressed in terms of Hironaka's weak equivalence class. Recall here that the weak equivalence class of $f_{n} W^{n}$ is defined in the context of $k$-algebras of finite type, whereas the local rings $\mathcal{O}_{V^{(d)}, x}$ are not within this class.

The claim is straightforward when the point $x$ is closed. As this function is not upper semicontinuous, it requires some clarification if $x \in \operatorname{Sing}\left(f_{n} W^{n}\right)$ is not a closed point. Let $Y$ denote the variety with generic point $x$. Since the weak equivalence class allows restriction to open sets, we may assume that $Y$ is smooth.

Fix a closed point $p$ at $Y$ and fix local coordinates $\left\{z, x_{2}, \ldots, x_{d}\right\}$ at $\mathcal{O}_{V^{(d)}, p}$, so that $\left\{x_{2}, \ldots, x_{d}\right\}$ is a regular system of parameters at $\mathcal{O}_{V^{(d-1)}, \beta(p)}$. We may assume, applying Lemma 4.2(2), the following:

(1) $I(Y)=\left\langle z, x_{2}, \ldots, x_{\ell}\right\rangle$.

(2) $f=z^{n}+a_{1} z^{n-1}+\cdots+a_{n}$.

(3) $I(\beta(Y))=\left\langle x_{2}, \ldots, x_{\ell}\right\rangle$.

In particular, $g r_{I(\beta(Y))}\left(\mathcal{O}_{V^{(d-1)}, \beta(p)}\right)=\mathcal{O}_{\beta(Y), \beta(p)}\left[X_{2}, \ldots, X_{\ell}\right]$. Set $\mathbf{x}=\beta(x)$, and let $k(\mathbf{x})$ be the quotient field of $\mathcal{O}_{\beta(Y), \beta(p)}$.

Note that $\mathbf{w}-\mathbf{i n}_{Y}\left(f_{n}\right) \in g r_{I(\beta(Y))}\left(\mathcal{O}_{V^{(d-1)}, \beta(p)}\right)$ can be naturally identified with $\mathbf{w}$-in $\mathbf{x}\left(f_{n}\right)$ via the inclusion $g r_{I(\beta(Y))}\left(\mathcal{O}_{V^{(d-1)}, \beta(p)}\right) \subset g r_{k(\mathbf{x})}\left(\mathcal{O}_{V^{(d)}, x}\right)$. In this setting, one can check that the sequences of transformations used in the previous proof to determine the rational number $q$ are expressed in terms of the weak equivalence class of $f_{n} W^{n}$. This proves the claim for the case in which $x$ is a non-closed point of $X$.

\section{Slope of a ReEs Algebra AND}

THE $(d-1)$-DIMENSIONAL H-FUNCTION $(\tau \geq 1)$

5.1. We rephrase the results and invariants discussed in Section 4 but now in the context of Rees algebras. Here, Theorem 5.10 parallels Theorem 4.10 in the previous section.

Throughout the section, we fix a Rees algebra $G=\bigoplus I_{n} W^{n}$ over $V^{(d)}$, and assume that $\boldsymbol{\tau}_{\mathcal{G}} \geq 1$ along closed points of $\operatorname{Sing}(G)$. Consider a transversal projection $V^{(d)} \stackrel{\beta}{\longrightarrow} V^{(d-1)}$, and assume in addition that $G$ is a $\beta$-differential Rees algebra (see 2.8).

Notation 5.1. Given two Rees algebras $G_{1}$ and $G_{2}$ defined over a smooth scheme $V$, we denote by $G_{1} \odot G_{2}$ the smallest Rees algebra containing both of them.

Proposition 5.2 (Local presentation). Fix a closed point $x \in \operatorname{Sing}(G)$ for wich $G$ is simple, and a locally defined $V^{(d)} \stackrel{\beta}{\rightarrow} V^{(d-1)}$, transversal to $G$ at $x$ (see 2.9). Assume that $G$ is a $\beta$-relative differential Rees algebra. Fix $f_{n} W^{n} \in G$ so that $f_{n}$ has order $n$ at $\mathcal{O}_{V^{(d)}, x}$. Assume that $f_{n}=f_{n}(z)$ is a monic polynomial of degree $n$ in $\mathcal{O}_{V^{(d-1)}, \beta(x)}[z]$, where $z$ is a $\beta$-section. Then, in a neighborhood of $x, \mathcal{G}$ has the 
same integral closure as

$$
\mathcal{O}_{V^{(d)}}\left[f_{n}(z) W^{n}, \Delta_{Z}^{j}\left(f_{n}(z)\right) W^{n-j}\right]_{1 \leq j \leq n-1} \odot \beta^{*}\left(\mathcal{R}_{\mathcal{G}, \beta}\right),
$$

where $\Delta_{z}^{j}$ are suitable $\beta$-differential operators of order $j$. Moreover, $\mathcal{R}_{\mathcal{G}, \beta}$ is non-zero whenever $\operatorname{Sing}(G)$ is not of codimension one in $V^{(d)}$.

Proof. See [6, Proposition 2.11].

Remark 5.3. The differential operators $\Delta_{z}^{j}$ in the previous proposition are the operators obtained by the Taylor morphism: this is a morphism of $S$-algebras (e.g., Tay $: S[Z] \longrightarrow S[Z, T]$ ), defined by setting $\operatorname{Tay}(Z)=Z+T$ (Taylor expansion). Here,

$$
\operatorname{Tay}(f(Z))=f(Z+T)=\sum \Delta^{r}(f(Z)) T^{r},
$$

and these operators $\Delta^{r}: S[Z] \longrightarrow S[Z]$ are defined by this morphism. It is well known that $\left\{\Delta^{0}, \Delta^{1}, \ldots, \Delta^{r}\right\}$ is a basis of the free module of $S$-differential operators of order $r$. The same applies here for $\mathcal{O}_{V^{(d-1)}}[z]$ : the set $\left\{\Delta_{z}^{0}, \Delta_{z}^{1}, \ldots, \Delta_{z}^{r}\right\}$ spans the sheaf of differential operators of order $r$ relative to the smooth morphism $\beta: V^{(d)} \longrightarrow V^{(d-1)}$. Moreover, as $V^{(d)}$ is étale over $V^{(d-1)} \times \mathbb{A}^{1}$, the previous set also generated Diff $\beta$ ( $\beta$-linear differential operators of order $r$ ).

Definition 5.4 (Presentations). Fix, after suitable restriction in étale topology, a projection $V^{(d)} \stackrel{\beta}{\longrightarrow} V^{(d-1)}$ transversal to a simple $\beta$-relative differential Rees algebra $G$. Assume that $\operatorname{Sing}(G)$ has no components of codimension one and that the following apply:

(i) There is a $\beta$-section $z$ (or global section so that $\{\mathrm{d} z\}$ is a basis of the locally free module of $\beta$-differentials, e.g., $\left.\Omega_{\beta}^{1}\right)$.

(ii) There is an element $f_{n}(z) W^{n} \in \mathcal{G}$, where $f_{n}(z)$ is a monic polynomial of order $n$, for instance,

$$
f_{n}(z)=z^{n}+a_{1} z^{n-1}+\cdots+a_{n} \in \mathcal{O}_{V^{(d-1)}}[z],
$$

where each $a_{i}$ is a global function on $V^{(d-1)}$.

In this case, Proposition 5.2 holds; namely, $G$ has the same integral closure as

$$
\mathcal{O}_{V^{(d)}}\left[f_{n}(z) W^{n}, \Delta_{z}^{j}\left(f_{n}(z)\right) W^{n-j}\right]_{1 \leq j \leq n-1} \odot \beta^{*}\left(\mathcal{R}_{\mathcal{G}, \beta}\right) .
$$

Here, we say that $\beta: V^{(d)} \longrightarrow V^{(d-1)}$, the $\beta$-section $z$, and the polynomial $f_{n}(z)=z^{n}+a_{1} z^{n-1}+\cdots+a_{n}$ define a presentation of $G$. These data will be denoted by

$$
\mathcal{P}\left(\beta: V^{(d)} \longrightarrow V^{(d-1)}, z, f_{n}(z)=z^{n}+a_{1} z^{n-1}+\cdots+a_{n}\right),
$$

or simply by $\mathcal{P}\left(\beta, z, f_{n}(z)\right)$. 
Definition 5.5. Fix a Rees algebra $G$ and a presentation $\mathcal{P}\left(\beta, z, f_{n}(z)\right)$. Define the slope of $G$ relative to $\mathcal{P}$ at a point $y \in V^{(d-1)}$ as

$$
\begin{aligned}
\operatorname{SI}(\mathcal{P})(y) & :=\min _{1 \leq j \leq n}\left\{\frac{v_{y}\left(a_{j}\right)}{j}, \operatorname{ord}\left(\mathcal{R}_{G, \beta}\right)(y)\right\} \\
& =\min \left\{\operatorname{Sl}\left(f_{n}(z) W^{n}\right)(y), \operatorname{ord}\left(\mathcal{R}_{G, \beta}\right)(y)\right\} .
\end{aligned}
$$

Remark 5.6. A change of variables of the form $z_{1}=z+\alpha, \alpha \in \mathcal{O}_{V^{(d-1)}}$ (a global section of $V^{(d-1)}$ ), gives rise to a new presentation defined in a natural way, for instance, $\mathcal{P}_{1}=\mathcal{P}_{1}\left(\beta, z_{1}, f_{n}^{\prime}\left(z_{1}\right)\right)$.

Assume first that $\operatorname{Sl}(\mathcal{P})(y)=\operatorname{Sl}\left(f_{n}(z) W^{n}\right)(y)<\operatorname{ord}\left(\mathcal{R}_{G, \beta}\right)(y)$. In this case, there is a change of the form $z_{1}=z+\alpha$, so that the new presentation $\mathcal{P}_{1}$ has bigger slope at $y$ if and only if $\mathbf{w}$-in $\mathbf{n}_{y}\left(f_{n}(z)\right)$ is an $n$-th power (see Remark 4.6).

On the other hand, if $\operatorname{Sl}(\mathcal{P})(y)=\operatorname{ord}\left(\mathcal{R}_{G, \beta}\right)(y)$, the slope at $y$ cannot increase by any change of the presentation of this type.

Definition 5.7. Fix a Rees algebra $G$. A presentation $\mathcal{P}=\mathcal{P}\left(\beta, z, f_{n}(z)\right)$ is said to be in normal form at $y \in V^{(d-1)}$ if one of the following two conditions holds:

- Either $\operatorname{Sl}(\mathcal{P})(y)=\operatorname{ord}\left(\mathcal{R}_{\mathcal{G}, \beta}\right)(y)$,

- Or $\operatorname{Sl}(\mathcal{P})(y)=\operatorname{Sl}\left(f_{n}(z) W^{n}\right)(y)<\operatorname{ord}\left(\mathcal{R}_{\mathcal{G}, \beta}\right)(y)$ and $\mathbf{w}-\operatorname{in}_{y}\left(f_{n}(z)\right)$ is not an $n$-th power.

Remark 5.8. Fix, as in Definition 5.2, a Rees algebra $G$ and a transversal projection $V^{(d)} \stackrel{\beta}{\longrightarrow} V^{(d-1)}$, and assume now that we fix a point $y \in \beta(\operatorname{Sing}(G))$. Suppose that we are given two different presentations $\mathcal{P}_{1}=\mathcal{P}_{1}\left(\beta, z, f_{n}(z)\right)$ and $\mathcal{P}_{2}=\mathcal{P}_{2}\left(\beta, z^{\prime}, g_{m}\left(z^{\prime}\right)\right)$, both in normal form at $y$. Theorem 5.10 will show that

$$
\operatorname{Sl}\left(\mathcal{P}_{1}\right)(y)=\operatorname{Sl}\left(\mathcal{P}_{2}\right)(y) .
$$

Moreover, it will show that this rational number is indeed independent of the chosen projection $\beta$.

The following result parallels Proposition 4.8.

Proposition 5.9. Let $G$ be a Rees algebra, and let $\mathcal{P}=\mathcal{P}\left(\beta, z, f_{n}(z)\right)$ be a presentation. Fix a point $y \in V^{(d-1)}$, and assume that $\mathcal{P}$ is in normal form at $y$. Then, $y$ is a point of $\beta(\operatorname{Sing}(G))$ if and only if $\operatorname{Sl}(\mathcal{P})(y) \geq 1$.

Proof. First, assume that $\operatorname{SI}(\mathcal{P})(y) \geq 1$. Denote by $x \in V^{(d)}$ the unique point of the fiber $\beta^{-1}(y)$ defined by $z=0$. Fix a regular system of parameters $\left\{x_{1}, \ldots, x_{\ell}\right\}$ at $\mathcal{O}_{V^{(d-1)}, y}$. Since $\operatorname{Sl}_{y}(\mathcal{P}) \geq 1$, then $v_{y}\left(a_{j}\right) \geq j($ for $j=1, \ldots, n$ ) and $\operatorname{ord}\left(\mathcal{R}_{G, \beta}\right)(y) \geq 1$. Set $P=\left\langle z, x_{1}, \ldots, x_{\ell}\right\rangle$, and note that $f_{n}(z) \in P^{n}$. Check now that $V\left(\left\langle z, x_{1}, \ldots, x_{\ell}\right\rangle\right) \subset \operatorname{Sing}(G)$ (see also Proposition 2.7(2)).

For the converse, assume that $y \in \beta(\operatorname{Sing}(G))\left(\subset \operatorname{Sing}\left(\mathcal{R}_{G, \beta}\right)\right)$. In this case, $\operatorname{ord}\left(\mathcal{R}_{G, \beta}\right)(y) \geq 1$. Since $\mathcal{P}$ is in normal form at $y$, one of the following two cases can occur: 
(1) $\operatorname{Sl}(\mathcal{P})(y)=\operatorname{ord}\left(\mathcal{R}_{G, \beta}\right)(y) \geq 1$.

(2) $\operatorname{Sl}(\mathcal{P})(y)=\operatorname{Sl}\left(f_{n}(z) W^{n}\right)(y)<\operatorname{ord}\left(\mathcal{R}_{\mathcal{G}, \beta}\right)(y)$ and $\mathbf{w}-\mathbf{i n}_{y}\left(f_{n}(z)\right)$ is not an $n$-th power.

Then, Proposition 4.8 ensures that $\operatorname{Sl}(\mathcal{P})(y) \geq 1$.

Theorem 5.10. Fix a Rees algebra $G$, and assume that $\tau_{G} \geq 1$ along closed points of $\operatorname{Sing}(G)$. Fix a point $x \in \operatorname{Sing}(G)$, and consider a presentation $\mathcal{P}=$ $\mathcal{P}\left(\beta, z, f_{n}(z)\right)$ that is in normal form at $\beta(x)$.

Then, the rational value $\operatorname{SI}(\mathcal{P})(\beta(x))$ is completely characterized by the weak equivalence class of $G$ in a neighborhood of $x$.

Proof. Recall that $\operatorname{Sl}(\mathcal{P})(\mathbf{x})=\min \left\{\operatorname{Sl}\left(f_{n}(z) W^{n}\right)(\mathbf{x}), \operatorname{ord}\left(\mathcal{R}_{\mathcal{G}, \beta}\right)(\mathbf{x})\right\}$. We proceed in two stages.

- First, assume that $q:=\operatorname{Sl}\left(f_{n}(z) W^{n}\right)(\mathbf{x})<\operatorname{ord}\left(\mathcal{R}_{G, \beta}\right)(\mathbf{x})$. In this case, we will argue as in Theorem 4.10 to prove our claim. In our coming discussion, we make use of the fact that the transformation law of $\mathcal{R}_{\mathcal{G}, \beta}$ is the law defining Rees algebras (see (2.11)).

Fix the same notation as in the proof of Theorem 4.10, and consider $N$ monoidal transformations at $p_{0}, p_{1}, \ldots, p_{N-1}$ followed by $\ell_{N}$ transformations at codimension 2, where

$$
\ell_{N}= \begin{cases}\lfloor N(q-1)-1\rfloor & \text { if } N q \notin \mathbb{Z}, \\ N(q-1)-1 & \text { if } N q \in \mathbb{Z} .\end{cases}
$$

We claim that the highest possible number of transformations defined by blowingup centers of codimension 2 (in the sense of Stage B) is exactly this number $\ell_{N}$ (which is completely characterized by $N$ and $q$ ).

The sequence of $N+\ell$ monoidal transformations

$$
\begin{aligned}
& \begin{array}{cccc}
\mathcal{G}^{\prime} & G_{1} & \mathcal{G}_{N} & \mathcal{G}_{N+1} \\
V^{(d)} \times \mathbb{A}^{1} \stackrel{\pi_{p_{0}}}{\longleftarrow} V_{1}^{(d+1)} \stackrel{\pi_{p_{1}}}{\longleftarrow} \cdots \stackrel{\pi_{p_{N-1}}}{\longleftarrow} V_{N}^{(d+1)} \longleftarrow V_{N+1}^{(d+1)} \longleftarrow \cdots
\end{array} \\
& G_{N+\ell} \\
& \cdots \longleftarrow V_{N+\ell}^{(d+1)}
\end{aligned}
$$

(see (2.12)) gives rise to

$$
\begin{aligned}
& V^{(d-1)} \times \mathbb{A}^{1} \longleftarrow V_{1}^{(d)} \longleftarrow \cdots \longleftarrow V_{N}^{(d)} \longleftarrow \\
& \mathcal{R}_{G, \beta}^{\prime} \quad\left(\mathcal{R}_{G, \beta}\right)_{1} \quad\left(\mathcal{R}_{G, \beta}\right)_{N} \\
& V_{N+1}^{(d)} \longleftarrow \cdots \longleftarrow V_{N+\ell}^{(d)} \\
& \left(\mathcal{R}_{G, \beta}\right)_{N+1} \quad\left(\mathcal{R}_{G, \beta}\right)_{N+\ell}
\end{aligned}
$$


together with morphisms $\tilde{\beta}_{i}: V_{i}^{(d+1)} \longrightarrow V_{i}^{(d)}$ (see (4.9)). In addition, each $\left(\mathcal{R}_{\mathcal{G}, \beta}\right)_{i}$ is also defined as the elimination algebra of $\mathcal{G}_{i}$ via $\beta_{i}$ (see (3) in (2.16)).

Denote by $H$ the exceptional hypersurface introduced by the last of these transformations. Set $\left(\mathcal{R}_{\mathcal{G}, \beta}\right)^{\prime}=\left(\mathcal{R}_{G, \beta}\right)_{N+\ell}$. Check that our assumption ensures that the slope at the generic point of $H$ (e.g., $\left.\xi_{H}\right)$ is

$$
\operatorname{Sl}\left(\mathcal{P}^{\prime}\right)\left(\xi_{H}\right)=\operatorname{Sl}\left(f_{n}^{\prime}(z) W^{n}\right)\left(\xi_{H}\right)<\operatorname{ord}\left(\left(\mathcal{R}_{G, \beta}\right)^{\prime}\right)\left(\xi_{H}\right),
$$

where $\mathcal{P}^{\prime}=\mathcal{P}^{\prime}\left(\beta^{\prime}, z^{\prime}, f_{n}^{\prime}\left(z^{\prime}\right)\right)$ is a presentation given by the transform at $V_{N+\ell}^{(d+1)}$.

In the case where $N q \notin \mathbb{Z}$, we have

$$
0<\operatorname{Sl}\left(\mathcal{P}^{\prime}\right)\left(\xi_{H}\right)=\operatorname{Sl}\left(f_{n}^{\prime}\left(z^{\prime}\right) W^{n}\right)\left(\xi_{H}\right)<1,
$$

so $H$ is not a component of $\beta^{\prime}\left(\operatorname{Sing}\left(G^{\prime}\right)\right)$ (see Proposition 5.9 and Remark 4.3(3)).

On the contrary, if we assume now that $N q \in \mathbb{Z}$, then

$$
\operatorname{Sl}\left(\mathcal{P}^{\prime}\right)\left(\xi_{H}\right)=\operatorname{Sl}\left(f_{n}^{\prime}\left(z^{\prime}\right) W^{n}\right)=0 .
$$

Remark 4.5 ensures that $\mathbf{w}$-in $\xi_{H}\left(f_{n}^{\prime}\left(z^{\prime}\right)\right)$ can be identified with the equation defining the fiber over $\xi_{H}$, that is, $\left.f_{n}^{\prime}\left(z^{\prime}\right)\right|_{H}$, which can be naturally identified with $\mathbf{w}$-in $\mathbf{n}_{\mathbf{x}}\left(f_{n}(z)\right)$. The hypothesis ensures that $\mathbf{w}$-in $\mathbf{i n}_{\mathbf{x}}\left(f_{n}(z)\right)$ is not an $n$-th power, so Proposition 5.9 and Remark 4.3(3) apply here to show that $H$ is not a component of $\beta^{\prime}\left(\operatorname{Sing}\left(G^{\prime}\right)\right)$.

- Second, to finish the proof, assume now that $\operatorname{Sl}(\mathcal{P})(\mathbf{x})=\operatorname{ord}\left(\mathcal{R}_{\mathcal{G}, \beta}\right)(\mathbf{x})$. Check that $\operatorname{Sl}\left(\mathcal{P}^{\prime}\right)\left(\xi_{H}\right)=\operatorname{ord}\left(\left(\mathcal{R}_{G, \beta}\right)^{\prime}\right)\left(\xi_{H}\right)<1$ after the transformations indicated before, and hence that $H$ is not a component of $\beta^{\prime}\left(\operatorname{Sing}\left(G^{\prime}\right)\right)$.

The previous discussion shows that the value $\operatorname{Sl}(\mathcal{P})(\beta(x))$ is totally characterized by the weak equivalence class of $G$ by arguing as was done in (4.11).

Corollary 5.11. Let $G$ be a Rees algebra. Assume that $\tau_{\mathcal{G}} \geq 1$ along a closed point of $\operatorname{Sing}(G)$. Fix a point $x \in \operatorname{Sing}(\mathcal{G})$, and consider two different presentations $\mathcal{P}_{1}=\mathcal{P}\left(\beta_{1}, z_{1}, f_{1}\left(z_{1}\right)\right)$ and $\mathcal{P}_{2}=\mathcal{P}\left(\beta_{2}, z_{2}, f_{2}\left(z_{2}\right)\right)$ that are in normal form at $\beta_{1}(x)$ and $\beta_{2}(x)$, respectively. Then,

$$
\operatorname{Sl}\left(\mathcal{P}_{1}\right)\left(\beta_{1}(x)\right)=\operatorname{Sl}\left(\mathcal{P}_{2}\right)\left(\beta_{2}(x)\right) .
$$

Proof. The proof follows straightforwardly from Theorem 5.10.

The previous discussion leads to the following definition.

Definition 5.12. Fix a Rees algebra $G$, and assume that $\tau \geq 1$ along $\operatorname{Sing}(G)$. A function with rational values, called the $(d-1)$-dimensional H-function, for instance,

$$
\text { H-ord }^{(d-1)}(G)(-): \operatorname{Sing}(G) \longrightarrow \mathbb{Q}_{\geq 0},
$$


is defined by setting

$$
\begin{aligned}
\text { H-ord }^{(d-1)}(G)(x) & :=\operatorname{SI}(\mathcal{P})(\beta(x)) \\
& =\min _{1 \leq j \leq n}\left\{\frac{v_{\beta(x)}\left(a_{j}\right)}{j}, \operatorname{ord}\left(\mathcal{R}_{\mathcal{G}, \beta}\right)(\beta(x))\right\}
\end{aligned}
$$

at any point $x \in \operatorname{Sing}(G)$, where $\mathcal{P}=\mathcal{P}\left(\beta, z, f_{n}(z)=z^{n}+a_{1} z^{n-1}+\cdots+a_{n}\right)$ is a presentation in normal form at $\beta(x)$.

Corollary 5.11 (or Theorem 5.10) ensures that the previous function is well defined. Namely, it is intrinsic to $\mathcal{G}$, with independence of $\beta$ and of the presentations. Further corollaries can be stated as follows.

\section{Corollary 5.13.}

(1) Recall that two Rees algebras $G$ and $G^{\prime}$ with the same integral closure are also weakly equivalent. In particular, $\operatorname{Sing}(G)=\operatorname{Sing}\left(G^{\prime}\right)$, and both $H$ functions coincide; that is, $\mathrm{H}$-ord ${ }^{(d-1)}(G)(x)=\mathrm{H}$-ord ${ }^{(d-1)}\left(G^{\prime}\right)(x)$ at any point $x \in \operatorname{Sing}(G)=\operatorname{Sing}\left(G^{\prime}\right)$.

(2) A similar statement holds for the Rees algebras $G$ and $\operatorname{Diff}(G)$ (see 2.8). In fact, both are weakly equivalent; thus, at any point $x \in \operatorname{Sing}(G)=$ $\operatorname{Sing}(\operatorname{Diff}(G))$,

$$
\begin{gathered}
\text { H-ord }^{(d-1)}(G)(x)=\text { H-ord }^{(d-1)}(\operatorname{Diff}(G))(x) . \\
\text { 6. SIMPLIFIED PRESENTATIONS AND } \\
\text { THE }(d-r) \text {-DIMENSIONAL H-FUNCTIONS }(\tau \geq r)
\end{gathered}
$$

6.1. Let $G$ be a differential Rees algebra over $V^{(d)}$, as defined in 2.8. Fix a closed point $x \in \operatorname{Sing}(G)$, and assume that $\tau_{\mathcal{G}, x} \geq r$. A notion of presentations was introduced in Definition 5.4 for the case $r=1$ in terms of suitable morphisms $V^{(d)} \longrightarrow V^{(d-1)}$. These presentations were, in turn, the tool that enabled us to define the $\mathrm{H}$-functions in the $(d-1)$-dimensional case: namely, $\mathrm{H}$-ord ${ }^{(d-1)}(\mathcal{G})(x)$. In this section, we address the general case $\tau_{G, x} \geq r$. In 6.2, we initiate the discussion of presentations that will lead to the definition of a function in terms of a smooth morphism $V^{(d)} \stackrel{\beta}{\longrightarrow} V^{(d-r)}$. In Theorem 6.5 , it is proved that such presentations can be chosen in a simplified form, called simplified presentation. The generalized Weierstrass Preparation Theorem to be discussed in this section will be the key tool for this simplification.

The lower-dimensional H-functions will be introduced in Definition 6.10. They will appear as the most natural extension of Definition 5.12 to the case $r>1$. The value H-ord ${ }^{(d-r)}(\mathcal{G})(x)$ will be defined, first, in terms of simplified presentations, and finally Theorem 6.9(1) will prove that this value is an invariant, and hence that it is independent of any choice.

These functions will lead to some applications in singularity theory which will be addressed in Section 7. 
6.2. The case $\tau \geq 2$. Let $G$ be a differential Rees algebra. Fix a closed point $x \in \operatorname{Sing}(\mathcal{G})$. Suppose $\tau_{\mathcal{G}, x} \geq 2$, and fix a transversal projection $V^{(d)} \stackrel{\beta}{\longrightarrow} V^{(d-2)}$ (see 2.9). We will proceed essentially in two steps. We shall first indicate how to construct a factorization of the form

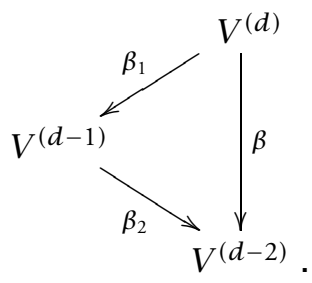

This diagram will allow us to define a coarse presentation in the setting of (2.24). We then proceed in a second step to construct a suitable change of the previous factorization of $\beta$. This last step will finally enable us to define a simplified presentation in the sense of (2.25).

In this first step, we make use of [4, Theorem 6.4], which ensures that if $\tau_{G, x} \geq 2$, then $\tau_{\mathcal{R}_{G, \beta_{1}}, \beta_{1}(x)} \geq 1$. This will enable us to apply twice Proposition 5.2. Note here that the existence of $\beta_{1}$ and $\beta_{2}$ together with 5.2 will provide us with a coarse presentation, so that $G$ will have the same integral closure as the algebra

$$
\begin{aligned}
\mathcal{O}_{V^{(d)}} & {\left[h_{\ell}\left(z_{1}\right) W^{\ell}, \Delta_{z_{1}}^{j_{1}}\left(h_{\ell}\left(z_{1}\right)\right) W^{\ell-j_{1}}, g_{m}\left(z_{2}\right) W^{m}, \Delta_{z_{2}}^{j_{2}}\left(g_{m}\left(z_{2}\right)\right) W^{m-j_{2}}\right]_{\substack{1 \leq j_{1} \leq \ell-1 \\
1 \leq j_{2} \leq m-1}} } \\
\odot \beta^{*}\left(\mathcal{R}_{G, \beta}\right) &
\end{aligned}
$$

(see Notation 5.1), where

$$
h_{\ell}\left(z_{1}\right) \in \mathcal{O}_{V^{(d-1)}}\left[z_{1}\right] \quad \text { and } \quad g_{m}\left(z_{2}\right) \in \mathcal{O}_{V^{(d-2)}}\left[z_{2}\right]
$$

are monic polynomials on the transversal sections $z_{1}$ and $z_{2}$, respectively. Let us draw attention to the fact that the coefficients of $h_{\ell}\left(z_{1}\right)$ are in $\mathcal{O}_{V^{(d-1)}}$, whereas we aim to define a notion of slope involving polynomials with coefficients in $\mathcal{O}_{V^{(d-2)}}$ (simultaneous elimination of two variables).

This is the second step addressed in the next proposition. It is proved that, locally in étale topology, there is a simplified presentation, so that $G$ and

$$
\begin{aligned}
\mathcal{O}_{V^{(d)}} & {\left[f_{n}^{\prime}\left(z_{1}^{\prime}\right) W^{n}, \Delta_{z_{1}^{\prime}}^{j_{1}}\left(f_{n}^{\prime}\right) W^{n-j_{1}}, g_{m}^{\prime}\left(z_{2}^{\prime}\right) W^{m}, \Delta_{z_{2}^{\prime}}^{j_{2}}\left(g_{m}^{\prime}\right) W^{m-j_{2}}\right]_{\substack{1 \leq j_{1} \leq n-1 \\
1 \leq j_{2} \leq m-1}} } \\
\odot \beta^{*}\left(\mathcal{R}_{\mathcal{G}, \beta}\right) &
\end{aligned}
$$

have the same integral closure, where

$$
f_{n}^{\prime}\left(z_{1}^{\prime}\right) \in \mathcal{O}_{V^{(d-2)}}\left[z_{1}^{\prime}\right] \quad \text { and } \quad g_{m}^{\prime}\left(z_{2}^{\prime}\right) \in \mathcal{O}_{V^{(d-2)}}\left[z_{2}^{\prime}\right]
$$


are monic polynomials on sections $z_{1}^{\prime}$ and $z_{2}^{\prime}$, respectively. Note that now both are polynomials with coefficients in $\mathcal{O}_{V^{(d-2)}}$. The strategy used to accomplish this second step will be done by changing the factorization of $\beta$ in (6.1).

Proposition 6.1. Let $\mathcal{G}$ be a differential Rees algebra, and let $x \in \operatorname{Sing}(G)$ be a closed point at which $\tau_{\mathcal{G}, x} \geq 2$. Then, at a suitable neighborhood of $x$, a transversal morphism (e.g., $\left.V^{(d)} \stackrel{\beta}{\longrightarrow} V^{(d-2)}\right)$ can be constructed in such a way that the following hold:

(1) There are global sections $z_{1}, z_{2}$, and $\left\{\mathrm{d} z_{1}, \mathrm{~d} z_{2}\right\}$ is a basis of the module of $\beta$-differentials, for instance, $\Omega_{\beta}^{1}$;

(2) There are two elements $f_{n} W^{n}, g_{m} W^{m} \in G$ of the form

$$
\begin{aligned}
& f_{n}\left(z_{1}\right)=z_{1}^{n}+a_{1} z_{1}^{n-1}+\cdots+a_{n} \in \mathcal{O}_{V^{(d-2)}, \beta(x)}\left[z_{1}\right], \\
& g_{m}\left(z_{2}\right)=z_{2}^{m}+b_{1} z_{2}^{m-1}+\cdots+b_{m} \in \mathcal{O}_{V^{(d-2)}, \beta(x)}\left[z_{2}\right],
\end{aligned}
$$

and $G$ has the same integral closure as

$$
\begin{aligned}
\mathcal{O}_{V^{(d)}} & {\left[f_{n}\left(z_{1}\right) W^{n}, \Delta_{z_{1}}^{\alpha}\left(f_{n}\right) W^{n-\alpha}, g_{m}\left(z_{2}\right) W^{m}, \Delta_{z_{2}}^{\gamma}\left(g_{m}\right) W^{m-\gamma}\right]_{\substack{1 \leq \alpha \leq n-1 \\
1 \leq \gamma \leq m-1}} } \\
& \odot \beta^{*}\left(\mathcal{R}_{G, \beta}\right) .
\end{aligned}
$$

Here, $\mathcal{R}_{G, \beta}$ denotes the elimination algebra, and $\Delta_{z_{i}}^{j}$ are as in Remark 5.3. In addition, $\mathcal{R}_{G, \beta}$ is non-zero whenever $\operatorname{Sing}(G)$ is not of codimension two locally at $x$.

Definition 6.2 (Simplified presentations for $\tau \geq 2$ ). Let the setting be as above. We say that

$$
s \mathcal{P}\left(\beta, z_{1}, z_{2}, f_{n}\left(z_{1}\right), g_{m}\left(z_{2}\right)\right)
$$

defines a simplified presentation of $G$.

Idea of the proof of Proposition 6.1. Fix $x \in \operatorname{Sing}(G)$, so that $\tau_{G, x} \geq 2$. We will first indicate how to produce a diagram as (6.1). Once this task is achieved, we will construct a scheme $V_{2}^{(d-1)}$, and two smooth morphisms $V^{(d)} \stackrel{\delta_{1}}{\rightarrow} V_{2}^{(d-1)}$ and $V_{2}^{(d-1)} \stackrel{\delta_{2}}{\longrightarrow} V^{(d-2)}$, so that the following diagram commutes:

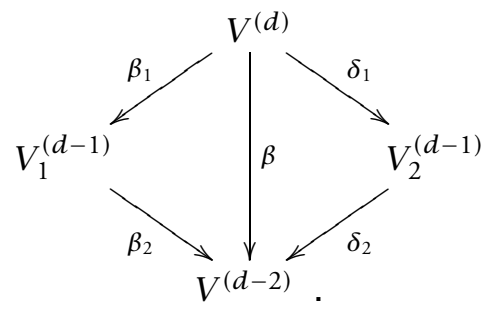

The triangle in the right-hand side will be better suited for our purpose as we will see in the following steps. 
Step 1: We shall first construct a morphism $\beta: V^{(d)} \longrightarrow V^{(d-2)}$ together with a diagram (6.1), and with the following condition:

- There is an element $h_{\ell} W^{\ell} \in G$ so that $h_{\ell}$ is a monic polynomial on $z$ of degree $\ell$, for instance,

$$
h_{\ell}(z)=z^{\ell}+c_{1} z^{\ell-1}+\cdots+c_{\ell} \in \mathcal{O}_{V_{1}^{(d-1)}, \beta_{1}(x)}[z],
$$

where $z$ is a global section, so that $\{\mathrm{d} z\}$ is a basis of the module of $\beta_{1}$ differentials.

Denote by $\mathcal{R}_{G, \beta_{1}}\left(\subset \mathcal{O}_{V_{1}^{(d-1)}}[W]\right)$ the elimination algebra corresponding to $\beta_{1}$. This is a simple algebra at $\beta_{1}(x) \in \operatorname{Sing}\left(\mathcal{R}_{G, \beta_{1}}\right)$. We know that $\tau_{\mathcal{R}_{G}, \beta_{1}}, \beta_{1}(x) \geq 1$, since $\tau_{G, x} \geq 2$, as was previously indicated. Therefore, we have the following:

There is an element $g_{m} W^{m} \in \mathcal{R}_{\mathcal{G}, \beta_{1}}$ so that $g_{m}$ is a monic polynomial of degree $m$, for instance,

$g_{m}\left(z_{2}\right)=z_{2}^{m}+b_{1} z_{2}^{m-1}+\cdots+b_{m} \in \mathcal{O}_{V^{(d-2)}, \beta(x)}\left[z_{2}\right]$, where $z_{2}$ is a global section and $\left\{\mathrm{d} z_{2}\right\}$ is a basis of the module of $\beta_{2}$-differentials. Hence, $\left\{\mathrm{d} z, \mathrm{~d} z_{2}\right\}$ is a basis of the module of $\beta$-differentials.

Step 2: Here, we will address the construction of a smooth scheme $V_{2}^{(d-1)}$, together with morphisms $V^{(d)} \stackrel{\delta_{1}}{\longrightarrow} V_{2}^{(d-1)}$ and $V_{2}^{(d-1)} \stackrel{\delta_{2}}{\longrightarrow} V^{(d-2)}$, so as to complete the diagram (6.4). The functions $f_{n}\left(z_{1}\right)$ and $g_{m}\left(z_{2}\right)$, with the conditions specified in Proposition 6.1, will arise from the construction of the right-hand side in (6.4).

Step 1 provides us with an element $g_{m}\left(z_{2}\right) W^{m} \in \mathcal{R}_{G, \beta_{1}}$. Via the natural inclusion $\mathcal{R}_{\mathcal{G}, \beta_{1}} \subset \mathcal{G}$ (see Proposition 2.7(1)), the element $\boldsymbol{g}_{m}\left(z_{2}\right) W^{m} \in \mathcal{G}$. The smooth scheme $V_{2}^{(d-1)}$ will be constructed below so as to dominate $V^{(d-2)}$ and so that $z_{2} \notin \mathcal{O}_{V_{2}^{(d-1)}}$. Moreover, a smooth morphism $\delta_{1}: V^{(d)} \longrightarrow V_{2}^{(d-1)}$ will be constructed with the condition that $g_{m}\left(z_{2}\right) \in \mathcal{O}_{V_{2}^{(d-1)}}\left[z_{2}\right]$, and that $z_{2}$ defines a section for $\delta_{1}$. Here, $\left\{\mathrm{d} z_{2}\right\}$ will be a basis for the module of $\delta_{1}$-differentials.

As $\boldsymbol{\tau}_{\mathcal{G}, x} \geq 2$, we know that $\boldsymbol{\tau}_{\mathcal{R}_{G, \delta_{1}}, \delta_{1}(x)} \geq 1$ ([4, Theorem 6.4]). This ensures the existence of the following:

an element $f_{n} W^{n} \in \mathcal{R}_{G}, \delta_{1}$ which is a monic polynomial, that is,

$$
f_{n}\left(z_{1}\right)=z_{1}^{n}+a_{1} z_{1}^{n_{1}-1}+\cdots+a_{n} \in \mathcal{O}_{V^{(d-2)}}\left[z_{1}\right] \text {, }
$$
where again $z_{1}$ is a global section so that $\left\{\mathrm{d} z_{1}\right\}$ is a basis of $\Omega_{\delta_{2}}^{1}$, and hence $\left\{\mathrm{d} z_{1}, \mathrm{~d} z_{2}\right\}$ defines a basis of $\Omega_{\beta}^{1}$.

Finally, check that if we succeed in the development of the previous steps, then $f_{n}\left(z_{1}\right)$ and $g_{m}\left(z_{2}\right)$ will fulfill the condition of the Proposition 6.1. 
Proof. Here, we construct (6.4) with the previously required conditions. Étale topology will be used throughout this proof, so let us specify some well-known properties of étale maps.

Fix a smooth scheme $V$, and suppose we are given a scheme $W$ and a smooth morphism

$$
\underset{W}{\downarrow} .
$$

This setting is preserved in étale topology when an étale map $W^{\prime} \stackrel{e}{\longrightarrow} W$ is considered. In fact, a commutative diagram arises by taking fiber products:

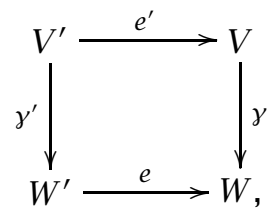

where $V^{\prime} \stackrel{e^{\prime}}{\longrightarrow} V$ is an étale map, and $V^{\prime} \stackrel{\gamma^{\prime}}{\longrightarrow} W^{\prime}$ is smooth. This says that the construction of a scheme $W$ and a smooth morphism $\gamma$ is preserved in étale topology, but only when lifting étale maps in the previous sense (from down-up). This will be the key point for the construction of the schemes and morphisms previously mentioned. Recall our general strategy:

Step 1: First, construct the left-hand side of (6.4), namely, the smooth schemes $V_{1}^{(d-1)}, V^{(d-2)}$ and the morphisms $\beta_{1}$ and $\beta_{2}$ with the required conditions.

Step 2: Once the previous data is fixed, complete the diagram (6.4) (the righthand side) in such a way that the polynomials $f_{n}\left(z_{1}\right)$ and $g_{m}\left(z_{2}\right)$ can be chosen as in Proposition 6.1.

To show this, we proceed in two stages:

(1) By assumption $\boldsymbol{\tau}_{\mathcal{G}, x} \geq 2(\geq 1)$, one can find a regular system of parameters $\left\{x_{1}, \ldots, x_{d}\right\}$ at $\mathcal{O}_{V^{(d)}, x}$, and an element $h_{\ell} W^{\ell} \in \mathcal{G}$ so that

$$
\left.h_{\ell}\right|_{x_{1}=\cdots=x_{d-1}=0}=u \cdot x_{d}^{\ell}
$$

for some unit $u \in \mathcal{O}_{V^{(d)}} /\left\langle x_{1}, \ldots, x_{d-1}\right\rangle$.

Consider the smooth morphism

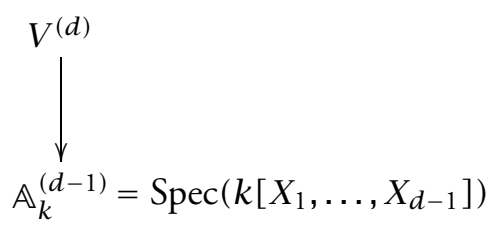


defined by $X_{i} \mapsto x_{i}$ for $i=1, \ldots, d-1$. Let $(B, N)$ be the henselization of the local ring $k\left[X_{1}, \ldots, X_{d-1}\right]_{\left\langle X_{1}, \ldots, X_{d-1}\right\rangle}$. This defines $\operatorname{Spec}(B) \rightarrow \mathbb{A}_{k}^{(d-1)}$. Up to multiplication by a unit, the element $h_{\ell}$ is a monic polynomial of degree $\ell$, that is,

$$
h_{\ell}(z)=z^{\ell}+c_{1} z^{\ell-1}+\cdots+c_{\ell} \in B[z] .
$$

Now, replace $\mathbb{A}_{k}^{(d-1)}$ by a suitable étale neighborhood $V_{1}^{(d-1)}$ where all the coefficients $c_{i}$ are global sections. Define $\beta_{1}$ by taking the fiber product. We abuse the notation and set $\beta_{1}: V^{(d)} \longrightarrow V_{1}^{(d-1)}$.

Let $\mathcal{R}_{G, \beta_{1}}$ denote the elimination algebra with respect to $\beta_{1}$. Since we have $\tau_{\mathcal{G}, x} \geq 2$, then again $\tau_{\mathcal{R}_{G, \beta_{1}}, \beta_{1}(x)} \geq 1$, and we repeat the previous argument to define a scheme (e.g., $V^{(d-2)}$ ) together with a smooth morphism $\beta_{2}$, so that a given element $g_{m} W^{m} \in \mathcal{R}_{G, \beta_{1}}$ of order $m$ can be expressed as a monic polynomial

$$
g_{m}\left(z_{2}\right)=z_{2}^{m}+b_{1} z_{2}^{m-1}+\cdots+b_{m} \in \mathcal{O}_{V^{(d-2)}}\left[z_{2}\right] .
$$

This construction might force us to replace the previous scheme $V_{1}^{(d-1)}$ by an étale neighborhood; in particular, $V^{(d)}$ is replaced by an étale neighborhood. Set $\beta=\beta_{2} \circ \beta_{1}$.

(2) Fix a regular system of parameters $\left\{x_{1}, \ldots, x_{d-2}\right\}$ at $\mathcal{O}_{V^{(d-2)}, \beta(x)}$. It extends to $\left\{x_{1}, \ldots, x_{d-2}, z, z_{2}\right\}$, which is a regular system of parameters at $\mathcal{O}_{V^{(d)}, x}$. Set $\mathbb{A}^{1}=\operatorname{Spec}(k[T])$. We now define a smooth morphism

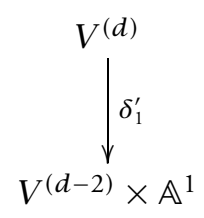

with the condition that $\operatorname{pr}_{1} \circ \delta_{1}^{\prime}$ yields $\beta=\beta_{2} \circ \beta_{1}$; here, $\mathrm{pr}_{1}$ is the projection in the first coordinate. The map $\delta_{1}^{\prime}$ is uniquely determined if $T$ is identified with the element $z$. Note that $\mathcal{O}_{V^{(d-2)}} \subset \mathcal{O}_{V^{(d-2)} \times \mathbb{A}^{1}}$, and hence $g_{m}\left(z_{2}\right) \in \mathcal{O}_{V^{(d-2)} \times \mathbb{A}^{1}}\left[z_{2}\right]$. Moreover, check that $z_{2}$ is a global section so that $\left\{\mathrm{d} z_{2}\right\}$ defines a basis of $\Omega_{\delta_{1}^{\prime}}^{1}$.

Let $\mathcal{H}:=\mathcal{R}_{G, \delta_{1}^{\prime}} \subset \mathcal{O}_{V^{(d-2)} \times \mathbb{A}^{1}}[W]$ denote the elimination algebra with respect to $\delta_{1}^{\prime}$. Again, since $\boldsymbol{\tau}_{\mathcal{G}, x} \geq 2$, then $\boldsymbol{\tau}_{\mathcal{H}, \delta_{1}^{\prime}(x)} \geq 1$. The same arguments used before ensure that the following exist:

- an étale neighborhood of $V^{(d-2)}$, say $V^{\prime(d-2)}$;

- a smooth morphism $V_{2}^{(d-1)} \stackrel{\delta_{2}}{\longrightarrow} V^{\prime(d-2)}$ (here, $V_{2}^{(d-1)}$ is an étale neighborhood of $\left.V^{(d-2)} \times \mathbb{A}^{1}\right)$;

- an element $f_{n} W^{n} \in \mathcal{H}(\subset \mathcal{G})$ that can be expressed as

$(* *) \quad f_{n}\left(z_{1}\right)=z_{1}^{n}+a_{1} z_{1}^{n_{1}-1}+\cdots+a_{n} \in \mathcal{O}_{V^{\prime}(d-2)}\left[z_{1}\right]$,

with the required properties. 
This settles construction of diagram (6.4), and the two polynomials $f_{n}\left(z_{1}\right)$ and $g_{m}\left(z_{2}\right)$ fulfill the conditions of Proposition 6.1.

Definition 6.3. Fix a Rees algebra $G$ so that $\tau_{G, x} \geq 2$ at any closed point $x \in \operatorname{Sing}(G)$, and a simplified presentation, for instance,

$$
s \mathcal{P}=s \mathcal{P}\left(\beta, z_{1}, z_{2}, f_{n_{1}}\left(z_{1}\right), f_{n_{2}}\left(z_{2}\right)\right),
$$

as in (6.3). The slope of $G$ relative to $s \mathcal{P}$ at a point $y \in V^{(d-2)}$ is defined as

$$
\operatorname{Sl}(s \mathcal{P})(y):=\min _{\substack{1 \leq j_{1} \leq n_{1} \\ 1 \leq j_{2} \leq n_{2}}}\left\{\frac{v_{y}\left(a_{j_{1}}\right)}{j_{1}}, \frac{v_{y}\left(b_{j_{2}}\right)}{j_{2}}, \operatorname{ord}\left(\mathcal{R}_{G, \beta}\right)(y)\right\} .
$$

Here, $v_{y}$ denotes the order at the regular local ring $\mathcal{O}_{V^{(d-2)}, y}$, and ord $\mathcal{R}_{\mathcal{G}, \beta}$ is the order function of the Rees algebra $\mathcal{R}_{G, \beta}$ as defined in (2.17).

Definition 6.4. Let $G$ be a Rees algebra, so that $\tau_{G, x} \geq 2$ for all $x \in \operatorname{Sing}(G)$. A simplified presentation $s \mathcal{P}=s \mathcal{P}\left(\beta, z_{1}, z_{2}, f_{n_{1}}\left(z_{1}\right), f_{n_{2}}\left(z_{2}\right)\right)$ is said to be in normal form at $y \in V^{(d-2)}$ if one of the following conditions hold:

- Either $\operatorname{Sl}(s \mathcal{P})(y)=\operatorname{ord}\left(\mathcal{R}_{G, \beta}\right)(y)$,

- Or $\operatorname{Sl}(s \mathcal{P})(y)=\operatorname{Sl}\left(f_{n_{1}}\left(z_{1}\right) W^{n_{1}}\right)(y)<\operatorname{ord}\left(\mathcal{R}_{G, \beta}\right)(y)$ and the weighted initial form $\mathbf{~}-\mathbf{i n}_{y}\left(f_{n_{1}}\left(z_{1}\right)\right)$ is not an $n_{1}$-th power,

- $\operatorname{Or} \operatorname{Sl}(s \mathcal{P})(y)=\operatorname{Sl}\left(f_{n_{2}}\left(z_{2}\right) W^{n_{2}}\right)(y)<\operatorname{ord}\left(\mathcal{R}_{G, \beta}\right)(y)$ and the weighted initial form $\mathbf{w}$-in $y\left(f_{n_{2}}\left(z_{2}\right)\right)$ is not an $n_{2}$-th power.

It will be shown in Theorem 6.9 that the rational number $\operatorname{Sl}(s \mathcal{P})(y)$ is entirely determined by the weak equivalence class of $G$, whenever $s \mathcal{P}$ is in normal form at $y \in \beta(\operatorname{Sing}(G))$. This will lead to the definition of an $\mathrm{H}$-function along points of $\operatorname{Sing}(G)$.

6.3. The general case $\tau \geq e$. We address here the case $\tau \geq e$, now for arbitrary $e$. It parallels the previous results for $e=2$, and generalizes, to some extent, the Weierstrass Preparation Theorem.

Theorem 6.5. Let $\mathcal{G}$ be a Rees algebra such that $\tau_{\mathcal{G}, x} \geq e$ at a closed point $x \in \operatorname{Sing}(G)$. Then, at a suitable étale neighborhood of $x$, a transversal morphism, for instance, $V^{(d)} \stackrel{\beta}{\longrightarrow} V^{(d-e)}$, can be defined together with the following:

- global functions $z_{1}, \ldots, z_{e}$ such that $\left\{\mathrm{d} z_{1}, \ldots, \mathrm{d} z_{e}\right\}$ forms a basis of $\Omega_{\beta}^{1}$ (module of $\beta$-relative differentials);

- integers $n_{1}, \ldots, n_{e} \in \mathbb{Z}_{>0}$;

- elements $f_{n_{1}} W^{n_{1}}, \ldots, f_{n_{e}} W^{n_{e}} \in \mathcal{G}$, where

$$
f_{n_{1}}\left(z_{1}\right)=z_{1}^{n_{1}}+a_{1}^{(1)} z_{1}^{n-1}+\cdots+a_{n_{1}}^{(1)} \in \mathcal{O}_{V^{(d-e)}}\left[z_{1}\right]
$$

$$
f_{n_{e}}\left(z_{e}\right)=z_{e}^{n_{e}}+a_{1}^{(e)} z_{r}^{n_{e}-1}+\cdots+a_{n_{e}}^{(e)} \in \mathcal{O}_{V^{(d-e)}}\left[z_{e}\right]
$$


(for some $a_{i}^{(j)}$ global functions on $\left.V^{(d-e)}\right)$. Moreover, the previous data can be defined with the condition that $G$ has the same integral closure as

$$
\mathcal{O}_{V^{(d)}}\left[f_{n_{i}}\left(z_{i}\right) W^{n_{i}}, \Delta_{z_{i}}^{j_{i}}\left(f_{n_{i}}\left(z_{i}\right)\right) W^{n_{i}-j_{i}}\right]_{\substack{1 \leq j_{i} \leq n_{i}-1 \\ i=1, \ldots, e}} \odot \beta^{*}\left(\mathcal{R}_{\mathcal{G}, \beta}\right) .
$$

Here, $\mathcal{R}_{G, \beta} \subset \mathcal{O}_{V^{(d-e)}}[W]$ is the elimination algebra defined in terms of $\beta$ as in Proposition 2.7, and $\Delta_{z_{i}}^{j}$ are as in Remark 5.3.

Proof. The proof follows from a natural extension of the arguments in the proof of Proposition 6.1.

Definition 6.6. (Simplified presentations). Let the setting be as in Theorem 6.5. The data

$$
s \mathcal{P}\left(\beta, z_{1}, \ldots z_{e}, f_{n_{1}}\left(z_{1}\right), \ldots, f_{n_{e}}\left(z_{e}\right)\right)
$$

that fulfills the conditions of Theorem 6.5 are said to be a simplified presentation.

The following definitions extend those in 6.3 and 6.4.

Definition 6.7. Let $G$ be a differential Rees algebra $G$ so that $\tau_{G, x} \geq e$ at a closed point $x \in \operatorname{Sing}(\mathcal{G})$. Fix, at a neighborhood of $x$, a simplified presentation, for instance, $s \mathcal{P}=s \mathcal{P}\left(\beta, z_{1}, \ldots, z_{e}, f_{n_{1}}\left(z_{1}\right), \ldots, f_{n_{e}}\left(z_{e}\right)\right)$, as in (6.7). The slope of $\mathcal{G}$ relative to $s \mathcal{P}$ at a point $y \in V^{(d-e)}$ is the rational number defined as

$$
\operatorname{Sl}(s \mathcal{P})(y):=\min _{\substack{1 \leq j_{i} \leq n_{i} \\ 1 \leq i \leq e}}\left\{\frac{v_{y}\left(a_{j_{i}}^{(i)}\right)}{j_{i}}, \operatorname{ord}\left(\mathcal{R}_{\mathcal{G}, \beta}\right)(y)\right\} .
$$

Definition 6.8. Let $G$ be a differential Rees algebra in the conditions of the previous definition. A simplified presentation $s \mathcal{P}=s \mathcal{P}\left(\beta, z_{i}, f_{n_{i}}\left(z_{i}\right)\right)_{1 \leq i \leq e}$ is said to be in normal form at $y \in V^{(d-e)}$ if one of the following conditions holds:

- Either $\operatorname{Sl}(s \mathcal{P})(y)=\operatorname{ord}\left(\mathcal{R}_{G, \beta}\right)(y)$,

- Or $\operatorname{Sl}(s \mathcal{P})(y)=\operatorname{Sl}\left(f_{n_{i}}\left(z_{i}\right) W^{n_{i}}\right)(y)<\operatorname{ord}\left(\mathcal{R}_{\mathcal{G}, \beta}\right)(y)$ for some index $1 \leq i \leq e$, and $\mathbf{w}$-in $\mathbf{n}_{y}\left(f_{n_{i}}\left(z_{i}\right)\right)$ is not an $n_{i}$-th power.

The next theorem will show that, given a simplified presentation $s \mathcal{P}$ in normal form at $y \in \beta(\operatorname{Sing}(G))$, the rational value $\operatorname{Sl}(s \mathcal{P})(y)$ is an invariant (independent of the presentation).

Theorem 6.9. Let $G$ be a $\beta$-differential Rees algebra (e.g., a differential Rees algebra) with the property that $\tau_{\mathcal{G}} \geq e$ along closed points in $\operatorname{Sing}(G)$. Fix a point $x \in \operatorname{Sing}(G)$, and assume that there is a simplified presentation $s \mathcal{P}$ that is in normal form at $\beta(x)$ (see Definition 6.8).

(1) The rational number $q=\operatorname{Sl}(s \mathcal{P})(\beta(x))$ in (6.8) is entirely determined by the weak equivalence class of $G$.

(2) $\mathcal{G}$ and $\mathcal{O}_{V^{(d)}}\left[f_{n_{1}} W^{n_{1}}, \ldots, f_{n_{r}} W^{n_{e}}\right] \odot \beta^{*}\left(\mathcal{R}_{\mathcal{G}, \beta}\right)$ are weakly equivalent. 
Proof.

(1) The proof is similar to that of Theorem 5.10, if we consider now, in Stage B, blow-ups at centers of codimension $e+1$ instead of codimension 2 .

(2) Theorem 6.5 ensures that $s \mathcal{P}$ can be chosen so that $\mathcal{G}$ has the same integral closure as

$$
\mathcal{G}_{1}=\mathcal{O}_{V^{(d)}}\left[f_{n_{i}}\left(z_{i}\right) W^{n_{i}}, \Delta_{z_{i}}^{j_{i}}\left(f_{n_{i}}\left(z_{i}\right)\right) W^{n_{i}-j_{i}}\right]_{\substack{1 \leq j_{i} \leq n_{i}-1 \\ i=1, \ldots, e}} \odot \beta^{*}\left(\mathcal{R}_{G, \beta}\right) .
$$

Theorem 3.4(1) asserts that $G$ and $G_{1}$ are weakly equivalent. Finally, Theorem $3.4(2)$ ensures that $G_{1}$ and $\mathcal{O}_{V^{(d)}}\left[f_{n_{1}} W^{n_{1}}, \ldots, f_{n_{e}} W^{n_{e}}\right] \odot \beta^{*}\left(\mathcal{R}_{\mathcal{G}, \beta}\right)$ are weakly equivalent.

Definition 6.10. First, assume the conditions of Theorem 6.9. Then, the $(d-e)$-dimensional H-function H-ord ${ }^{(d-e)}(G): \operatorname{Sing}(G) \longrightarrow \mathbb{Q}_{\geq 0}$ is defined by setting

$$
{\mathrm{H}-\operatorname{ord}^{(d-e)}}^{(G)}(x)=\operatorname{Sl}(s \mathcal{P})(\beta(x)),
$$

where $s \mathcal{P}$ is a simplified presentation which is in normal form at $\beta(x)$.

Theorem 6.9 ensures this value is well defined, namely, that it is independent of the choice of the smooth morphism $\beta$ and of the simplified presentation.

\section{Main Properties of H-Functions and Proof of THEOREM 2.13}

7.1. A particular kind of simplified presentations: $p$-presentations. The form of elimination of a variable we use here is largely sustained on the presence of a polynomial that is monic on this variable, namely, on the Weierstrass Preparation Theorem. Moreover, the elimination algebras that appear in our discussions are defined in terms of these monic polynomials. An example of this fact arises already in Theorem 6.5.

Of particular interest will be the case in which the degrees of these monic polynomials are given by powers of the characteristic: namely, when $n_{i}=p^{\ell_{i}}$ in (6.5), where $p$ denotes the characteristic of the underlying field. In fact, in this case, there is a surprising interplay between the coefficients of these polynomials and the elimination algebra, which we will indicate below.

Fix a $d$-dimensional scheme $V^{(d)}$ smooth over a perfect field $k$ together with a differential Rees algebra (e.g., $G$ ) over $V^{(d)}$. Fix a transversal projection $V^{(d)} \stackrel{\beta}{\longrightarrow}$ $V^{(d-1)}$. As $G$ is a differential algebra, it is also a $\beta$-differential algebra (see 2.9). In this case, we will indicate that, locally at any point $x \in \operatorname{Sing}(G)$, the $\beta$-differential structure of $\mathcal{G}$ will allow us to consider a simplified presentation $s \mathcal{P}$ with integers of the form $n_{1}=p^{\ell_{1}} \leq n_{2}=p^{\ell_{2}} \leq \cdots \leq n_{e}=p^{\ell_{e}}$, where $p$ denotes 
the characteristic of $k$. These particular simplified presentations will be called $p$-presentations, and will be denoted by

$$
p \mathcal{P}=p \mathcal{P}\left(\beta, z_{i}, f_{p^{\ell_{i}}}\left(z_{i}\right)=z_{i}^{p^{\ell_{i}}}+a_{1}^{(i)} z^{p^{\ell_{i}}-1}+\cdots+a_{p^{\ell_{i}}}\right)_{1 \leq i \leq e} .
$$

The exponents $\ell_{1} \leq \ell_{2} \leq \cdots \leq \ell_{e}$ are closely related to invariants studied by Hironaka in [34], and are also related to other invariants introduced by Kawanoue and Matsuki in [38], [39], and [40].

The notion of $p$-presentations was introduced in [6] for the case $e=1$. They were denoted by

$$
p \mathcal{P}=p \mathcal{P}\left(\beta: V^{(d)} \longrightarrow V^{(d-1)}, z, f_{p^{\ell}}(z)=z^{p^{\ell}}+a_{1} z^{p^{\ell}-1}+\cdots+a_{p^{\ell}}\right),
$$

and it was shown there that the following hold:

(1) Given $x \in \operatorname{Sing}(G)$, the $p$-presentation can be modified into a new $p$ presentation that is in normal form at $\beta(x)$ (see Definition 5.7).

(2) The value of the $\mathrm{H}$-function is given by

$$
\mathrm{H}-\operatorname{ord}^{(d-1)}(\mathcal{G})(x)=\min \left\{\frac{v_{\beta(x)}\left(a_{p^{\ell}}\right)}{p^{\ell}}, \operatorname{ord}\left(\mathcal{R}_{\mathcal{G}, \beta}\right)(\beta(x))\right\},
$$

where we have the right-hand side defined in terms of a $p$-presentation $p \mathcal{P}=p \mathcal{P}\left(\beta, z, f_{p^{\ell}}(z)\right)$ in normal form at $\beta(x)$ (see [6, Theorem 4.6 and Corollary A.9]).

This simplifies the expression in (5.4), as only the coefficient $a_{p^{\ell}}$ appears in this formulation. Surprisingly, the contribution of the intermediate coefficients of $f_{p^{\ell}}(z)=z^{p^{\ell}}+a_{1} z^{p^{\ell}-1}+\cdots+a_{p^{\ell}}$ is somehow encoded in the elimination algebra $\mathcal{R}_{G, \beta}$.

The previous two properties extend easily to the general case $\tau \geq e$ via Theorem 6.5 together with presentations as in (7.1). Namely, we have the following:

(1) Given $x \in \operatorname{Sing}(G)$, the $p$-presentation $p \mathcal{P}$ in (7.1) can be modified into another $p$-presentation, which is in normal form at $\beta(x)$. Let us emphasize here that $p$-presentations are particular forms of simplified presentations.

(2) Assume now that $p \mathcal{P}$ is in normal form at $\beta(x)$; then,

$$
\mathrm{H}-\operatorname{ord}^{(d-e)}(G)(x)=\min _{1 \leq j \leq e}\left\{\frac{v_{\beta(x)}\left(a_{p^{\ell_{j}}}^{(j)}\right)}{p^{\ell_{j}}}, \operatorname{ord}\left(\mathcal{R}_{G, \beta}\right)(\beta(x))\right\} \text {. }
$$

7.2. p-presentations and monoidal transformations. Thus far, $G$ has been assumed to be a differential Rees algebra. It is in this context in which Theorem 6.5 applies, namely, that the theorem ensures simplified presentations exist for these Rees algebras. 
A natural notion of transformation of a $p$-presentation was studied for the case $e=1$ in [6]. There, given a $p$-presentation of $G$ over $V^{(d)}$ (for instance, $\left.p \mathcal{P}=p \mathcal{P}\left(\beta, z, f_{p^{\ell}}\right)\right)$, a new $p$-presentation (e.g., $\left.p \mathcal{P}_{1}=p \mathcal{P}_{1}\left(\beta_{1}, z_{1}, f_{p^{\ell}}^{(1)}\right)\right)$ is defined in terms of $p \mathcal{P}$ and of the monoidal transformation. This is denoted by

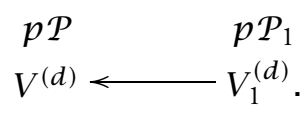

The simplified presentations given by Theorem 6.5 enables us to extend the previous discussion to the case $\tau \geq e$ for presentations in the setting of (7.1). In other words, the previous discussion applies for a presentation once we fix a sequence of transformations (2.16). This will enable us to attach a simplified presentation to $G_{r}$, locally at $x \in \operatorname{Sing}\left(G_{r}\right)$, in terms of $\beta_{r}$, and this will be achieved by successive "transformations."

The result in Theorem 6.9 has now a natural formulation for the $\beta_{r}$-differential Rees algebra $G_{r}$ in (2.16). In addition, Definition 6.10 can also be stated for $G_{r}$. Consequently, the $(d-e)$-dimensional H-function of $G_{r}$, for instance,

$$
\text { H-ord }^{(d-e)}\left(G_{r}\right): \operatorname{Sing}\left(G_{r}\right) \longrightarrow \mathbb{Q}_{\geq 0},
$$

can be defined, and again Theorem 6.9 applies to ensure the function is intrinsic.

7.3. On tamed H-functions and the proof of Theorem 2.13. We address here the proof of Theorem 2.13. First, we introduce the notion of strong monomial case in Definition 7.1, which will encode the numerical conditions in (2.20). Once this point is settled, the statement of Theorem 2.13 will be reformulated in Theorem 7.3.

As a first step, recall the construction of the monomial algebra introduced in (2.19), where a monomial algebra, for instance,

$$
\mathcal{M}_{r} W^{S}=\mathcal{O}_{V_{r}^{(d-e)}}\left[I\left(H_{1}\right)^{h_{1}} \ldots I\left(H_{r}\right)^{h_{r}} W^{S}\right]
$$

is assigned to any sequence (2.16) by setting, for $i=0, \ldots, r-1$,

$$
\frac{h_{i+1}}{S}=\text { H-ord }^{(d-e)}\left(G_{i}\right)\left(\xi_{Y_{i}}\right)-1,
$$

and where $\xi_{Y_{i}}$ denotes the generic point of $Y_{i}$, the center of the monoidal transformation.

Definition 7.1. Let $G$ be a Rees algebra so that $\tau_{G}(x) \geq e$ at any closed point $x \in \operatorname{Sing}(G)$. Let the setting be as in 2.10, and assume that (2.16) is defined so that the elimination algebra of $G_{r}$ is monomial in the sense of (2.18). 
The Rees algebra $G_{r}$ is said to be in the strong monomial case if, at any closed point $x \in \operatorname{Sing}\left(G_{r}\right)$,

$$
\text { H-ord }{ }^{(d-e)}\left(G_{r}\right)(x)=\operatorname{ord}\left(\mathcal{M}_{r} W^{s}\right)(x) .
$$

Remark 7.2. In [6, Theorem 8.5 and Remark 8.6], it is proved that the condition $\operatorname{ord}\left(\mathcal{M}_{r} W^{s}\right)(x)=\mathrm{H}$-ord ${ }^{(d-1)}\left(G_{r}\right)(x)=\operatorname{ord}\left(\left(\mathcal{R}_{G, \beta}\right)_{r}\right)\left(\beta_{r}(x)\right)$ holds if and only if $\mathcal{M}_{r} W^{s}=\left(\mathcal{R}_{\mathcal{G}, \beta}\right)_{r}$, and this ensures the existence of a hypersurface of maximal contact locally at the point (case $e=1$ ). The extension of this fact to the general case is straightforward.

Theorem 7.3. Let the setting be as in Definition 7.1. Assume that $G_{r}$ is in the strong monomial case at any closed point $x \in \operatorname{Sing}\left(G_{r}\right)$. Then, any combinatorial resolution of $\mathcal{M}_{r} W^{s}$ can be lifted to a sequence of monoidal transformations over $G_{r}$, for instance,

$$
\begin{array}{ccccc}
G & G_{1} & G_{r} & G_{N} \\
V^{(d)} \stackrel{\pi_{Y}}{\longleftarrow} V_{1}^{(d)} \stackrel{\pi_{Y_{1}}}{\longleftarrow} \cdots \stackrel{\pi_{Y_{r-1}}}{\longleftarrow} V_{r}^{(d)} \stackrel{\pi_{Y_{r}}}{\longleftarrow} \cdots \stackrel{\pi_{Y_{N-1}}}{\longleftarrow} V_{N}^{(d)},
\end{array}
$$

which is either a resolution of $G$ (i.e., $\operatorname{Sing}(G)=\varnothing)$ or $\tau_{G_{N}}(x) \geq e+1$ at any closed point $x \in \operatorname{Sing}\left(G_{N}\right)$ (improvement of the $T$-invariant).

Proof. We consider here the situation where $\tau_{G_{r}, x} \geq e$ for all closed points $x \in \operatorname{Sing}\left(G_{r}\right)$, and we will study the closed points $x$ where $\tau_{G, x}=e$. Our proof will strongly rely on the existence of $p$-presentations (see 7.1), for instance, $s \mathcal{P}=s \mathcal{P}\left(\beta, z_{i}, f_{p^{\ell_{i}}}\left(z_{i}\right)=z_{i}^{p^{\ell_{i}}}+a_{1}^{(i)} z^{p^{\ell_{i}}-1}+\cdots+a_{p^{\ell_{i}}}\right)_{1 \leq i \leq e}$, which we may assume, in addition, are in normal form at $\beta(x)\left(x \in \operatorname{Sing}\left(G_{r}\right)\right)$.

First, assume, as we previously indicated, that the elimination algebra of $G_{r}$ is monomial, namely, that

$$
\left(\mathcal{R}_{G, \beta}\right)_{r}=\mathcal{O}_{V_{r}^{(d-e)}}\left[I\left(H_{1}\right)^{\alpha_{1}} \ldots I\left(H_{r}\right)^{\alpha_{r}} W^{s}\right] .
$$

The monomial algebra $\mathcal{M}_{r} W^{s}$ in (7.4) relates to the $p$-presentation in the following manner:

- $a_{j_{i}}^{(i)} W^{j_{i}} \in \mathcal{M}_{r} W^{s}$ for $j_{i}=1, \ldots, p^{\ell_{i}}, i=1, \ldots, e$. This means that the coefficients $a_{j_{i}}^{(i)} W^{j_{i}}$ are in the integral closure of $\mathcal{M}_{r} W^{s}$. We express this fact by saying that $\mathcal{M}_{r} W^{s}$ divides the coefficients.

- $\mathcal{M}_{r} W^{s}$ divides the elimination algebra $\left(\mathcal{R}_{G, \beta}\right)_{r}$ in (7.8) (i.e., $h_{i} \leq \alpha_{i}$ for $i=1, \ldots, r)$. This fact is guaranteed by (7.5) and (7.3), together with the law of transformations for Rees algebras.

Let us address now the numerical conditions defined in terms of the $\mathrm{H}$ functions $\mathrm{H}$-ord ${ }^{(d-e)}$ at points of $\operatorname{Sing}\left(G_{r}\right)$. First, recall the formulation of the $\mathrm{H}$-function in (7.3) that relies only on the elimination algebra or on the constant terms of the $e$ polynomials (e.g., $a_{p^{\ell_{i}}}^{(i)}$. 
The following two cases can occur:

(A) Assume that H-ord ${ }^{(d-e)}\left(G_{r}\right)(x)=\operatorname{ord}\left(\left(\mathcal{R}_{G, \beta}\right)_{r}\right)\left(\beta_{r}(x)\right)$ at $x \in \operatorname{Sing}\left(G_{r}\right)$. In this case, Remark 7.2 says that $\mathcal{M}_{r} W^{s}=\left(\mathcal{R}_{G, \beta}\right)_{r}$; this, in turn, ensures that all coefficients $a_{j_{i}}^{(i)} W^{j_{i}} \in\left(\mathcal{R}_{\mathcal{G}, \beta}\right)_{r}$. These conditions, together with the fact that $f_{p^{\ell_{i}}}\left(z_{i}\right) W^{p^{\ell_{i}}} \in \mathcal{G}_{r}$, say that, up to integral closure, $z_{i} W \in G_{r}$ for $i=1, \ldots, e$. In particular, this guarantees that $z_{1}=\cdots=z_{e}=0$ has maximal contact with $G_{r}$ at $x$, and hence in an open neighborhood of $x$. Therefore, at a neighborhood of $x$ the theorem can be dealt with as in the case of characteristic zero.

(B) Assume that $\mathrm{H}$-ord ${ }^{(d-e)}\left(G_{r}\right)(x)<\operatorname{ord}\left(\left(\mathcal{R}_{G, \beta}\right)_{r}\right)\left(\beta_{r}(x)\right)$ at $x \in \operatorname{Sing}\left(G_{r}\right)$. Then, there is an index $j$ for which

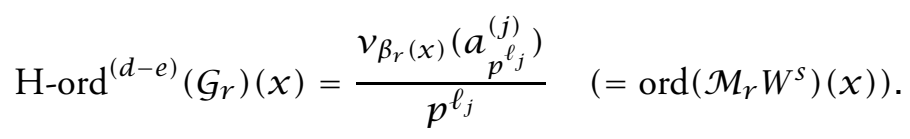

This equality shows that $a_{p^{\ell_{j}}}^{(j)} W^{p^{\ell_{j}}}=\mathcal{M}_{r} W^{s}$, meaning that both algebras have the same integral closure. This ensures that, up to multiplication by a unit, $a_{p^{\ell_{j}}}^{(j)}$ can be taken as a monomial.

Fix now a smooth permissible center $C$ with generic point $y$, so that $x \in C$. A property of $p$-presentations is that they can be chosen so as to be in normal form simultaneously at $x$ and $y$ (see [6, Proposition 5.8]). We now compute H-ord ${ }^{(d-e)}\left(G_{r}\right)(y)$. We claim that, for the same index $j$ as we have taken before,

$$
\text { H-ord }{ }^{(d-e)}\left(G_{r}\right)(y)=\frac{v_{\beta_{r}(y)}\left(a_{p^{\ell_{j}}}^{(j)}\right)}{p^{\ell_{j}}}
$$

In fact, $\operatorname{ord}\left(\mathcal{M}_{r} W^{s}\right)(y)=v_{\beta_{r}(y)}\left(a_{p^{\ell_{j}}}^{(j)}\right) / p^{\ell_{j}}$, and since all coefficients and the elimination algebra are divisible by $\mathcal{M}_{r} W^{s}$, we have

$$
\begin{aligned}
& \text { H-ord }{ }^{(d-e)}(G)(y)=\min _{1 \leq j \leq e}\left\{\frac{v_{\beta_{r}(y)}\left(a_{p^{\ell_{j}}}^{(j)}\right)}{p^{\ell_{j}}}, \operatorname{ord}\left(\left(\mathcal{R}_{G, \beta}\right)_{r}\right)\left(\beta_{r}(x)\right)\right\} \\
& =\frac{v_{\beta_{r}(y)}\left(a_{p^{\ell_{j}}}^{(j)}\right)}{p^{\ell_{j}}} \text {. }
\end{aligned}
$$

To summarize, there is a particular index $j$ with the following properties:

- $j$ provides the value of the H-function at $x$ and at $y$. Namely,

${\mathrm{H}-\operatorname{ord}^{(d-e)}}_{(\mathcal{G})}(x)=\frac{v_{\beta_{r}(x)}\left(a_{p^{\ell_{j}}}^{(j)}\right)}{p^{\ell_{j}}}$ and $\quad \mathrm{H}$-ord ${ }^{(d-e)}(\mathcal{G})(y)=\frac{v_{\beta_{r}(y)}\left(a_{p^{\ell_{j}}}^{(j)}\right)}{p^{\ell_{j}}}$.

- The role of this $j$ is stable under transformations. 
In this way, Theorem 6.5 reduces the proof of this theorem to the case of only one index (e.g., $j$ ), which leads us to consider the algebra

$$
G_{r}^{\prime}=\mathcal{O}_{V_{r}^{(d)}}\left[f_{p^{\ell_{j}}}\left(z_{j}\right) W^{p^{\ell_{j}}}, \Delta^{p^{\ell_{j}}-i}\left(f_{p^{\ell_{j}}}\left(z_{j}\right)\right) W^{p^{\ell_{j}-1}}\right]_{1 \leq i \leq p^{\ell_{j}-1}} \odot\left(\mathcal{R}_{G, \beta}\right)_{r} .
$$

Recall we assumed that $\tau_{G_{r}, x}=e$. The theorem of elimination of variables, together with the previous discussion, ensures that $\boldsymbol{T}_{\mathcal{G}_{r}^{\prime}, x}=1$, and moreover, that $G^{\prime}$ is in the strong monomial case. This is the setting in which [6, Theorem 8.14] applies; this last theorem is the particular case of Theorem 7.3, which applies for Rees algebras with $\tau$-invariant equal to 1 . It ensures that after a sequence of $N-r$ transformations, the final algebra $G_{N}^{\prime}$ is resolved, or $\tau_{\mathcal{G}_{N}^{\prime}, x^{\prime}} \geq 2$ for all $x^{\prime} \in \operatorname{Sing}\left(G_{N}^{\prime}\right)$. This sequence of transformations is permissible for $\mathcal{G}_{r}$. Moreover, it will guarantee that either $G_{N}$ is resolved, or $\tau_{G_{N}, x^{\prime \prime}} \geq e+1$ for all $x^{\prime \prime} \in \operatorname{Sing}\left(G_{N}\right)$.

\subsection{Application to embedded resolution of two-dimensional schemes.} We can now extend the embedded resolution of surfaces treated in [7] for the hypersurfaces case to prove embedded desingularization of arbitrary two-dimensional schemes (over perfect fields). This provides a desingularization of two-dimensional schemes a la Hironaka, namely, by applying successive monoidal transformations along the highest Hilbert-Samuel stratum.

Theorem 7.4. Given a reduced two-dimensional scheme $X$ over a perfect field, there is a sequence of monoidal transformations

$$
X \stackrel{\pi_{C_{1}}}{\stackrel{n}{\longleftarrow}} X_{1} \longleftarrow \cdots \stackrel{\pi_{C_{n}}}{=} X_{n},
$$

where each center of the blow-up $C_{i}$ is so that $C_{i} \subset \underline{\operatorname{Max}} H S_{X_{i}}$ (here, $\underline{\operatorname{Max}} H S_{X_{j}}$ denotes the maximum Hilbert-Samuel stratum of $X_{j}$ ), such that

(i) $X_{n}$ is a smooth embedded desingularization of $X$, which modifies only the singular points of $X$.

(ii) The exceptional locus of the composition map $X \stackrel{\pi}{\longleftarrow} X_{n}$ has only simple normal crossings.

Essential concepts of the proof.

(1) Consider an embedding of $X$ in a smooth $d$-dimensional scheme (e.g., $\left.X \subset V^{(d)}\right)$. In this setting, Hironaka attaches to the maximum HilbertSamuel stratum (e.g., Max $_{\mathrm{H}} S_{X}$ ) an algebra $G$.

(2) As $X$ is a two-dimensional scheme; then, $\tau_{\mathcal{G}, x} \geq d-2$ at any closed point of $x \in \operatorname{Sing}(G)=\underline{\operatorname{Max}} H_{X}$. See [10, Proposition 11.4].

(3) Our generalized Weierstrass Preparation Theorem in 6.5, and corresponding $\mathrm{H}$-functions in 6.10, let us extend the discussion in [7, Section 4].

Acknowledgements. We would like to thank the referee for suggestions that have helped us to improve the presentation of this paper. We have profited from discussions with K. Matsuki, A. Bravo, and S. Encinas.

The authors are partially supported by MTM2012-35849. 


\section{REFERENCES}

[1] Sh. ABHYANKAR, Local uniformization on algebraic surfaces over ground fields of characteristic $p \neq 0$, Ann. of Math. (2) 63 (1956), no. 3, 491-526. http://dx.doi.org/10.2307/1970014. MR0078017 (17,1134d)

[2] Uniformization in p-cyclic extensions of algebraic surfaces over ground fields of characteristic p, Math. Ann. 153 (1964), no. 2, 81-96. http://dx.doi.org/10.1007/BF01361 177. MR0159821 (28 \#3037)

[3] A. Altman and S. Kleiman, Introduction to Grothendieck Duality Theory, Lecture Notes in Mathematics, vol. 146, Springer Verlag, Berlin-New York, 1970. MR0274461 (43 \#224)

[4] A. Benito, The T-invariant and elimination, J. Algebra 324 (2010), no. 8, 1903-1920. http://dx.doi.org/10.1016/j.jalgebra.201 0.06.01 1. MR2678827 (2011i:14030)

[5] A. Benito, S. EnCinas, and O. E. Villamayor U., Some natural properties of constructive resolution of singularities, Asian J. Math. 15 (2011), no. 2, 141-191. http://dx.doi.org/10.4310/AJM.2011.v15.n2.a3. MR2838219

[6] A. BENITO and O. E. VIllamayOR U., Monoidal transforms and invariants of singularities in positive characteristic, Compos. Math. 149 (2013), no. 8, 1267-1311. http://dx.doi.org/10.1112/S0010437X1200084X. MR3103065

[7] - Techniques for the study of singularities with applications to resolution of 2-dimensional schemes, Math. Ann. 353 (2012), no. 3, 1037-1068.

http://dx.doi.org/10.1007/s00208-011-0709-5. MR2923956

[8] E. Bierstone and P. D. Milman, Canonical desingularization in characteristic zero by blowing up the maximum strata of a local invariant, Invent. Math. 128 (1997), no. 2, 207-302. http://dx.doi.org/10.1007/s002220050141. MR1440306 (98e:14010)

[9] A. BRAVO and O. E. VIllamayor U., Singularities in positive characteristic, stratification and simplification of the singular locus, Adv. Math. 224 (2010), no. 4, 1349-1418. http://dx.doi.org/10.1016/j.aim.2010.01.005. MR2646300 (2011j:14031)

[10] Elimination algebras and inductive arguments in resolution of singularities, Asian J. Math. 15 (2011), no. 3, 321-355. http://dx.doi.org/10.4310/AJM.2011.v15.n3.a2. MR2838211

[11] V. Cossart, Sur le polyèdre caractéristique, Thèse d'État, Univ. Paris Sud Orsay, 1987.

[12] V. Cossart, J. Giraud, and U. OrbanZ, Resolution of Surface Singularities, Lecture Notes in Mathematics, vol. 1101, Springer Verlag, Berlin, 1984. With an appendix by H. Hironaka. MR775681 (87e:14032)

[13] V. Cossart, U. JANnSEn, and S. SAITO, Canonical embedded and non-embedded resolution of singularities for excellent two-dimensional schemes (2009), preprint, available at http://arxiv.org/abs/0905.2191v1.

[14] V. COSSART and O. PILTANT, Resolution of singularities of threefolds in positive characteristic. I: Reduction to local uniformization on Artin-Schreier and purely inseparable coverings, J. Algebra 320 (2008), no. 2, 1051-1082. http://dx.doi.org/10.1016/j.jalgebra.2008.03.032. MR2427629 (2009f:14024)

[15] _ Resolution of singularities of threefolds in positive characteristic. II, J. Algebra 321 (2009), no. 7, 1836-1976.

http://dx.doi.org/10.1016/j.jalgebra.2008.1 1.030. MR2494751 (2010c:14009)

[16] Resolution of singularities of arithmetical threefolds I (2013), preprint, available at https://hal.archives-ouvertes.fr/hal-00873967.

[17] S. D. CUTKOSKY, Resolution of singularities for 3-folds in positive characteristic, Amer. J. Math. 131 (2009), no. 1, 59-127.

http://dx.doi.org/10.1353/ajm.0.0036. MR2488485 (2010e:14010)

[18] A A skeleton key to Abhyankar's proof of embedded resolution of characteristic $p$ surfaces, Asian J. Math. 15 (2011), no. 3, 369-416. http://dx.doi.org/10.4310/AJM.2011.v15.n3.a4. MR2838213 (2012m:14023)

[19] S. ENCINAS and H. HAUSER, Strong resolution of singularities in characteristic zero, Comment. Math. Helv. 77 (2002), no. 4, 821-845.

http://dx.doi.org/10.1007/PL00012443. MR1949115 (2004c:14021) 
[20] S. EnCinas and O. E. Villamayor U., A course on constructive desingularization and equivariance, Resolution of Singularities 1997 (Obergurgl), Progr. Math., vol. 181, Birkhäuser, Basel, 2000, pp. 147-227. MR1748620 (2001g:14018)

[21] _ A new proof of desingularization over fields of characteristic zero, Rev. Mat. Iberoamericana 19 (2003), no. 2, 339-353, Proceedings of the International Conference on Algebraic Geometry and Singularities (Spanish) (Sevilla, 2001).

http://dx.doi.org/1 0.4171/RMI/350. MR2023188 (2004m:14017)

[22] _ Rees algebras and resolution of singularities, Actas del "XVI Coloquio Latinoamericano de Algebra” (Colonia del Sacramento, Uruguay, 2005) (W. F. Santos, G. González-Springer, A. Rittatore, and A. Solotar, eds.), Library of the Revista Matemática Iberoamericana, 2007, pp. 124.

[23] J. GIRAUd, Contact maximal en caractéristique positive, Ann. Sci. École Norm. Sup. (4) 8 (1975), no. 2, 201-234 (French). MR0384799 (52 \#5671)

[24] - Forme normale d'une fonction sur une surface de caractéristique positive, Bull. Soc. Math. France 111 (1983), no. 2, 109-124 (French, with English summary). MR734215 (86h:14007)

[25] _ Condition de Jung pour les revêtements radiciels de hauteur 1, Algebraic Geometry 1982 (Tokyo/Kyoto), Lecture Notes in Math., vol. 1016, Springer, Berlin, 1983, pp. 313-333 (French). http://dx.doi.org/10.1007/BFb0099969. MR726432 (86d:14012)

[26] H. HAUSER, Kangaroo points and oblique polynomials in resolution of positive characteristic (December 18, 2008), preprint, available at http://arxiv.org/abs/arXiv:0811.4151.

[27] H. HAUSER and D. WAGNER, Two new invariants for the resolution of surfaces in positive characteristic, to appear.

[28] H. HIRONAKA, Resolution of singularities of an algebraic variety over a field of characteristic zero. I, Ann. of Math. (2) 79 (1964), no. 1, 109-203. http://dx.doi.org/10.2307/1970486. MR0199184 (33 \#7333)

[29] - Resolution of singularities of an algebraic variety over a field of characteristic zero. II, Ann. of Math. (2) 79 (1964), no. 2, 205-326. http://dx.doi.org/10.2307/1970547. MR0199184 (33\#7333)

[30] _ Introduction to the Theory of Infinitely Near Singular Points, Memorias de Matematica del Instituto "Jorge Juan", vol. 28, Consejo Superior de Investigaciones Científicas, Madrid, 1974. MR0399505 (53\#3349)

[31] _ Appendix: Desingularization of excellent surfaces, Advanced Science Seminar in Algebraic Geometry, Lecture Notes in Mathematics, vol. 1101, Springer Verlag, Berlin, 1984. (summer 1967 at Bowdoin College), Mimeographed notes by B. Bennet, pp. 99-132.

[32] __ Introduction to the Theory of Infinitely near Singular Points, Memorias de Matematica del Instituto "Jorge Juan", vol. 28, Consejo Superior de Investigaciones Científicas, Madrid, 1974. MR0399505 (53\#3349)

[33] _ Idealistic exponents of singularity, Algebraic Geometry (J.J. Sylvester Sympos., Johns Hopkins Univ., Baltimore, Md., 1976), Johns Hopkins Univ. Press, Baltimore, Md., 1977, pp. 52-125. MR0498562 (58 \#16661)

[34] - Additive groups associated with points of a projective space, Ann. of Math. (2) 92 (1970), no. 2, 327-334. http://dx.doi.org/10.2307/1970839. MR0269658 (42 \#4553)

[35] _ Introduction to the Theory of Infinitely near Singular Points, Memorias de Matematica del Instituto "Jorge Juan", vol. 28, Consejo Superior de Investigaciones Científicas, Madrid, 1974. MR0399505 (53\#3349)

[36] - Three key theorems on infinitely near singularities, Singularités Franco-Japonaises, Sémin. Congr., vol. 10, Soc. Math. France, Paris, 2005, pp. 87-126 (English, with English and French summaries). MR2145950 (2006i:14014)

[37] _ Program for Resolution of Singularities in Characteristics $p>0$ : Notes from Lectures at the Clay Mathematics Institute, September 2008.

[38] H. KaWANOUE, Toward resolution of singularities over a field of positive characteristic. I: Foundation; the language of the idealistic filtration, Publ. Res. Inst. Math. Sci. 43 (2007), no. 3, 819-909. http://dx.doi.org/1 0.2977/prims/1201012043. MR2361797 (2008m:14028) 
[39] H. KAWANOUE and K. MATSUKI, Toward resolution of singularities over a field of positive characteristic (the idealistic filtration program) Part II: Basic invariants associated to the idealistic filtration and their properties, Publ. Res. Inst. Math. Sci. 46 (2010), no. 2, 359-422. http://dx.doi.org/10.2977/prims/1201012043. MR2722782 (2011m:14024)

[40] _ Resolution of singularities of an idealistic filtration in dimension 3 after Benito-Villamayor, acepted for publication, available at http://arxiv.org/abs/arXiv:1205.4556v1.

[41] J. Lipman, Desingularization of two-dimensional schemes, Ann. Math. (2) 107 (1978), no. 2, 151-207. http://dx.doi.org/10.2307/1971141. MR0491722 (58 \#10924)

[42] T. T. MOH, On a stability theorem for local uniformization in characteristic $p$, Publ. Res. Inst. Math. Sci. 23 (1987), no. 6, 965-973. http://dx.doi.org/10.2977/prims/1 195175867. MR935710 (89d:14018)

[43] - On a Newton polygon approach to the uniformization of singularities of characteristic $p$, Algebraic Geometry and Singularities (La Rábida, 1991) (A. Campillo and L. Narvaéz, eds.), Progr. Math., vol. 134, Birkhäuser, Basel, 1996, pp. 49-93. MR1395176 (97d:14025)

[44] T. ODA, Infinitely very near singular points, Complex Analytic Singularities, Adv. Stud. Pure Math., vol. 8, North-Holland, Amsterdam, 1987, pp. 363-404. MR894302 (88j:14017)

[45] O. E. Villamayor U., Constructiveness of Hironaka's resolution, Ann. Sci. École Norm. Sup. (4) 22 (1989), no. 1, 1-32. MR985852 (90b:14014)

[46] _ Patching local uniformizations, Ann. Sci. École Norm. Sup. (4) 25 (1992), no. 6, 629677. MR1198092 (93m:14012)

[47] Rees algebras on smooth schemes: integral closure and higher differential operator, Rev. Mat. Iberoam. 24 (2008), no. 1, 213-242. http://dx.doi.org/1 0.4171/RMI/534. MR2435971 (2009f:14026)

[48] _. Hypersurface singularities in positive characteristic, Adv. Math. 213 (2007), no. 2, 687733. http://dx.doi.org/10.1016/j.aim.2007.01.006. MR2332606 (2008g:14021)

[49] Elimination with applications to singularities in positive characteristic, Publ. Res. Inst. Math. Sci. 44 (2008), no. 2, 661-697. http://dx.doi.org/10.2977/prims/1210167340. MR2426361 (2009h:14028)

[50] J. WŁodarczyK, Simple Hironaka resolution in characteristic zero, J. Amer. Math. Soc. 18 (2005), no. 4, 779-822 (electronic). http://dx.doi.org/1 0.1090/S0894-0347-05-00493-5. MR2163383 (2006f:14014)

[51] _ Program on Resolution of Singularities in Characteristic p: Notes from Lectures, RIMS, Kyoto, December 2008.

[52] O. Zariski and P. SAmuel, Commutative algebra. Vol. II, The University Series in Higher Mathematics, D. Van Nostrand Co., Inc., Princeton, N. J.-Toronto-London-New York, 1960. MR0120249 (22\#11006)

ANGÉLICA BENITO:

Department of Mathematics AND

University of Michigan

530 Church Street

Ann Arbor, MI 48109-1043, USA

E-MAIL: abenitos@umich.edu

Instituto de Ciencias Matemáticas (ICMAT)

C/ Nicolás Cabrera, no. 13-15

28049 Madrid, Spain

E-MAIL: angelica.benito@icmat.es

Orlando E. Villamayor U.:

Departamento de Matemáticas

Universidad Autónoma de Madrid and ICMAT-UAM

Ciudad Universitaria de Cantoblanco,

28049 Madrid, Spain

E-MAIL:villamayor@uam.es

KEY WORDS AND PHRASES: Singularities, positive characteristic, differential operators, Rees algebras.

2000 Mathematics Subject Classification: 14E15.

Received: September 26, 2013; revised: September 30, 2014. 